\author{
University of São Paulo \\ Ribeirão Preto Medical School \\ Department of Pathology and Forensic Medicine
}

(Brazil)

Differential mRNA and miRNA expression in oligodendrogliomas of different grades of malignancy
Expressão diferencial de RNAm e miRNAs em oligodendrogliomas de diferentes graus de malignidade

Muhammad Nawaz

Ribeirão Preto 


\title{
Differential mRNA and miRNA expression in oligodendrogliomas of different grades of malignancy
}

\begin{abstract}
Expressão diferencial de RNAm e miRNAs em oligodendrogliomas de diferentes graus de malignidade
\end{abstract}

A thesis submitted to Ribeirão Preto Medical School, University of São Paulo for the requirements to obtain doctorate of philosophy $(\mathrm{PhD})$.

Area of concentration: Experimental Pathology and Molecular Biology

Supervisor: Luciano Neder Serafini

Co-supervisor: Hadi Valadi

Ribeirão Preto 


\section{Autorizo a reprodução e divulgação total ou parcial deste trabalho, por qualquer meio convencional ou eletrônico, para fins de estudo e pesquisa, desde que citada a fonte.}

\section{Ficha Catalográfica}

Preparada pela Biblioteca Central do Campus Administrativo de Ribeirão Preto/USP

Nawaz, Muhammad

Differential mRNA and miRNA expression in oligodendrogliomas of different grades of malignancy. Ribeirão Preto, 2017.

131 p. : il. ; $30 \mathrm{~cm}$

Expressão diferencial de RNAm e miRNAs em oligodendrogliomas de diferentes graus de malignidade.

Tese de Doutorado, apresentada à Faculdade de Medicina de Ribeirão Preto/USP. Área de concentração: Biologia Molecular.

Orientador: Serafini, Luciano Neder.

Co-orientador: Valadi, Hadi.

1. Oligodendrogliomas 2. MicroRNA 3. mRNA 4. Differential expression 5. Bioinformatic analysis 6. RT-PCR 7. Exosomes 8. Microvesicles.

1. Oligodendrogliomas 2. MicroRNA 3. RNAm 4. Expressão diferencial 5. Análise bioinformática 6. RT-PCR 7. Exosomes 8. Microvesicles. 


\section{Declaration}

I hereby declare that the work presented in this thesis is my own effort and that the thesis is my own composition. I have not presented previously any part of the thesis for any other degree.

I authorize the reproduction of this work; by any means conventional or electronic, for study and research purposes, provided that the source is cited properly. 


\section{Financial support and resources}

The financial and academic support from the following resources made this work possible;

- São Paulo Research Foundation - FAPESP, Proc. No. 12/24574-3 (PhD scholarship)

- São Paulo Research Foundation - FAPESP, Proc. No. 08/05972-2 (Financial support)

- Coordination for the Improvement of Higher Education Personnel - CAPES (support for one year sandwich program at University of Gothenburg Sweden. Proc. No. BEX 7057/15-6)

- Coordination for the Improvement of Higher Education Personnel - CAPES (PhD scholarship in Brazil)

- Foundation for Teaching Support, and Research Assistance (FAEPA), from Hospital of Clinics, belonging to Ribeirão Preto Medical School (HC-FMRP, USP)

- Ribeirão Preto Medical School (FMRP, USP)

- Ribeirão Preto School of Pharmacy (FCFRP, USP)

- Department of Rheumatology and Inflammation Research (DRIR), Institute of Medicine, at Sahlgrenska Academy, University of Gothenburg, Sweden (hosted me for one year sandwich program)

I also acknowledge Adlerbert Foreign Student Hospitality award and the Adlerbertska Forskningsstiftelsen Research Foundation award from the University of Gothenburg, during my stay at Sweden. 
Name: Muhammad Nawaz

Title: Differential mRNA and miRNA expression in oligodendrogliomas of different grades of malignancy

Título: Expressão diferencial de RNAm e miRNAs em oligodendrogliomas de diferentes graus de malignidade
A thesis submitted to Ribeirão Preto Medical School, University of São Paulo for the requirements to obtain Doctorate of Philosophy in Experimental Pathology / Molecular Biology.

\section{Examination committee}

\section{President:}

Prof. Dr. Luciano Neder Serafini

Institution: FMRP/USP

Judgment:

Signatures

\section{Members:}

Prof. Dr. Suzana M. Fleury Malheiros

Institution: UNIFESP

Judgment:

Signatures

Prof. Dr. Jeremy A. Squire

Institution: FMRP/USP

Judgment:

Signatures

Prof. Dr. Fabio Rogerio

Institution: UNICAMP

Judgment:

Signatures 


\section{Dedication}

I dedicate this thesis to my loving parents who always been a continuous source of unconditional love, prayers and great support at every step of my life. 


\section{Personal Expressions}

I have learnt over the time that, courage of taking initiative, trying things out again and again, sometimes thinking out of the box and being reckless about outcomes is an inevitable key that take me to exceptional achievements. My innate habit of social interactions, expressing my words even of less importance; all have collectively made me socially enriched man. Setting out for journeys and the long travels have complimented social experience. Going through unusual ways, from trying small and silly things-to taking big decisions with affordable risks has concentrated me with leadership qualities over the time, and I am satisfied with what I do in life. 


\section{Acknowledgements}

All praise to Allah Almighty, the most beneficent, the most merciful, Who honoured man with intellectual power and spiritual insight to understand, discover and to conquer wondrous mysteries. Countless salutations upon the Holy Prophet Muhammad (peace be upon him), for enlightening humanity with the essence of faith and guiding the mankind to the true path of life.

I would like to express my profound gratitude to all those who helped me in different ways during my $\mathrm{PhD}$. In particular I am rather indebted to thank;

Luciano Neder: PhD supervisor, for giving me opportunity to work in his group. His kind supervision, guidance, and personal interests in my research work made this all possible. What most profoundly I recognize is that, he gave me freedom, letting me doing things independently with my own ideas and decisions. I took advantage of this freedom for writing fellowship applications independently, travel grants for participating in conferences and writing up scientific articles. Collectively this all made my way to academic growth as an independent researcher.

I have really enjoyed our chats and gossips not only about science, but about everything in life; family, culture and social norms across the borders. Many thanks for your support through difficult circumstances that had arisen throughout my stay at Ribeirao Preto. I really had an amazing time with you:-

Hadi Valadi: my co-supervisor, who proved to be one of the productive additions in my scientific career. It was a great experience being at Gothenburg and enjoying exosomes science in his lab at Sahlgrenska Academy. He provided me the facilities of the lab in a proper, suitable and helping manner and this was the first time I gained very authoritative knowledge about newly described area of exosomes research. This is safe to say that, it will come in very useful way in my future career. Last, but not least, my affiliations with him recognize his patronizing concerns towards scientific goals. There was so much to learn from you (-)

I thank Tao Jin at the Department of Rheumatology and Inflammation Research at Sahlgrenska Academy for his cooperation and support. And 'wow' all those guys at department lunch room with whom I have had company during lunch time:-

I cannot forget my past mentors Wasim Ahamd, Bushra Mirza, Muhammad Ansar, Salman Akbar Malik, Faqir Ahmad Khan, Tariq Mahmood for their exceptional contribution to my 
academics and strong impact on my personality development. I thank my all school and college/university teachers who contributed at various capacities in my academic life and career.

Jeremy Squire: Thank you so much for your prodigious scientific advises and dynamic encouragement. I learnt a lot from your vast experience in research and science. I enjoyed your company and scientific writings with you.

Alfredo Ribeiro da Silva and other members of the postgraduate program of department of pathology: for making things easy going, documentations and related matters at the department.

Sergio Albuquerque and Maria Vitoria Bentley (FCFRP directors), Victor Hugo, Elaine De Martinis, Márcia Eliana, Ana Patricia Yatsuda, Vanessa Muller and Henrique for their support at Ribeirão Preto School of Pharmacy, USP.

I thank Daniela Tirapeli and Fermino Sanches for their help and guidance while I spent few productive weeks in their lab. There much to learn from you, and of course nice company.

Many thanks to my lab colleagues; Bruna Zanetti, Niele Dias Mendes, Renata Silvestre, Nathalia and Isabela, Deise and Anabelle, for their pleasant company and assistance during my research work as well as helping through several difficulties that had arisen during my stay at Ribeirao Preto. Bruna and Renata you both have been very nice to me and my family.

I spent great time with Alexandros Papadimitriou and Marco Maugeri at University of Gothenburg. Thank you for all kind of cooperation at work bench as well as for the fun times. Alex had a perfect amount of sarcasm when needed; he was the guy keeping a friendly environment in the workplace. Thank you for nice chats and pleasant company.

I convey my heartiest and sincerest gestures to my Friends in Brazil and those at University of Sao Paul; Muhammad Tahir, Faheem Khan, Fazal Wahab, Farhad Ulluh, Mohd Jaseem, Yagoub Ali, Atlas Khan, Asmat Ullah, Guilherme Ferreira, Henrique Amaral, Jean Gonzales and Letícia Lima for their valuable time and pertinacious support during my stay at Brazil.

Camila Zambonini: Thank you so much for administrative help during all my time at this department. Without your support it was all hard to complete $\mathrm{PhD}$ (-) I also thank Rodrigo Banzi, Rosangela, Neide Terezinha and Antonio de Padua for helping me in several ways. Yes, that IT expert guy Felipe Denipotte Coelho: every time appears with a whistle and solves the technical issues(:). Thanks Felipe. 
Fernanda Bartolomeu and Fernanda Reis: Thank you very much for your help whenever I needed to prepare documentation for VISA and passport issues with federal legislative body. Every time, you helped in great way.

A quick appreciation to life at Brazil: while staying at Brazil, I found Brazilian people very kind, receptive and respectful. Natal and Carnival festivals, Samba music and Brazilian pizzamozzarella, one of its own kind, I loved it:-

Finally, my deepest gratitude goes to my parents for unconditional love and support throughout my life. I cannot unfold words to thank my parents for their endless love and affection; for whom there is no alternative in this world. They always stand beside me during all hardships and prayed for me at every occasion. They have both seen me cry, panic and laugh-through my childhood to adolescent, university life to married life, and they still manage to support me even through my most difficult times. I can never pay back any reward for their care.

My brothers (Muhammad Ijaz, Muhammad Shahbaz, Zafar ullah, Fakhar ullah and Qamar ullah and Ijaz Saleh), sisters and their families, my nephew Adil Mushtaq and relatives who are great support in my life; thank you all for your continuous support and prayers. Moreover, I extend special thanks to my in-laws for their help, continuous prayers and wishes for my success in life. May Allah keep my whole family and relatives, healthy and place them among His loved ones. Ameen

Last but not the least, my immense love and affection goes to my wife Farah Nawaz, who supported me at every hard time, shared workload, and gave me courage and motivation to step forward. She has been more like a friend and colleague, discussing science, writing scientific papers and travelling to new lands. We had best part of our life at Sweden a unique place to live. Thank you so much for your constant support. Our mutual understanding has matured over these years.

Our son, Muhammad Amad Shafi, who is the most precious gift from Allah, made tough times seem enjoyable and accompanied us on weekend experiments and conferences - giving us the best memories - .

Collectively, without encouragement and understanding of my family, it would have been impossible for me to keep going and all that what I have in my life. 


\section{CONTENTS}

List of abbreviations

List of figures

List of tables

\section{CHAPTER I}

1 INTRODUCTION 1

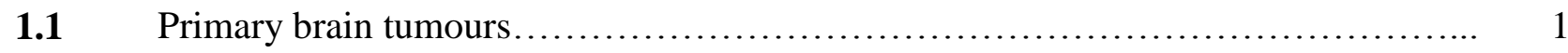

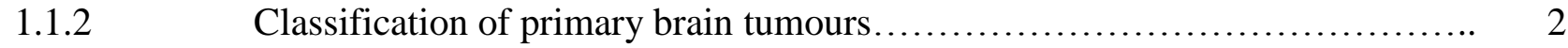

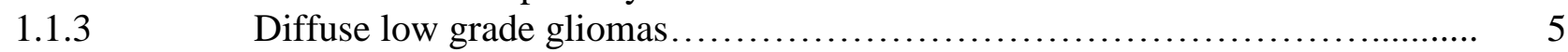

1.2 Oligodendrogliomas .................................................... 9

1.2.1 Molecular biology of oligodendrogliomas.............................. 10

1.2.1.1 IDH1 and TP53 Mutations........................................ 13

1.2.1.2 Mutations in CIC, FUBPlgenes and TERT promoter.......................... 14

1.2.1.3 Methylation of MGMT genes in oligodendrogliomas........................ 15

1.3 Genetics of $1 \mathrm{p} / 19 \mathrm{q}$ co-deletion and loss of heterozygosity....................... 20

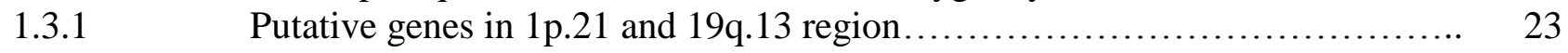

Clinical implications of $1 \mathrm{p}$ and $19 \mathrm{q}$ co-deletion........................... 23

$1.4 \quad$ Non coding RNAs (ncRNAs) ................................................... 26

1.4.1 MicroRNAs....................................................... 27

1.4.2 Roles in cancer initiation, progression and metastasis....................... 30

1.5 MicroRNAs in oligodendrogliomas..................................... 32

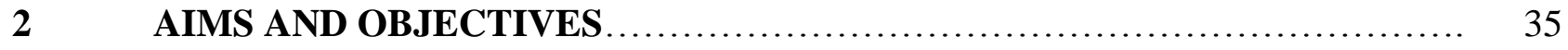

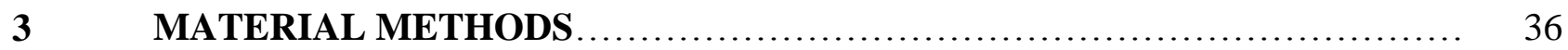

$3.1 \quad$ Patient samples...................................................... 36

Extraction and quantification of total RNA................................ 36

$3.3 \quad$ Microarrays for mRNA evaluation...................................... 36

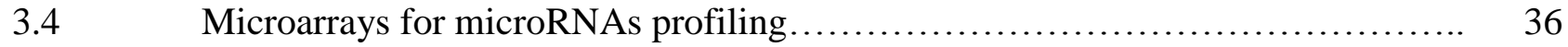

$3.5 \quad$ Analysis of microarray data................................................ 37

3.6 Differential expression analysis.......................................... 37

$3.7 \quad$ Determining microRNA:mRNA correlation ............................... 38

3.8 Validation of miRNA and gene expression by Real-Time PCR................. 38

3.9 Establishing primary cell lines of oligodendrogliomas....................... 38

Validation of miRNA and genes in cell lines.............................. 39

4 RESULTS.

4.1 Differentially expressed microRNAs.................................... 40

$4.2 \quad$ Differentially expressed mRNAs......................................... 41

4.3 Top differentially expressed miRNAs and mRNAs in grade II and III ODs........ 42

4.4 Assessment of differentially expressed miRNAs and mRNAs.................. 46

4.5 Mean miRNAs and mRNAs variations according the tumour grades.............. 49

Correlation between miRNAs and their mRNA-targets....................... 54 
4.7 Upregulated miRNAs and their respective mRNAs downregulated.............. 58

4.8 Downregulated miRNAs and their respective mRNAs upregulated.............. 61

V.9 Validation of miRNAs and mRNAs by RT-qPCR ......................... 61

Establishment of primary cells lines of oligodendrogliomas ................... 64

4.11 Validation of miRNAs in cell lines......................................... 66

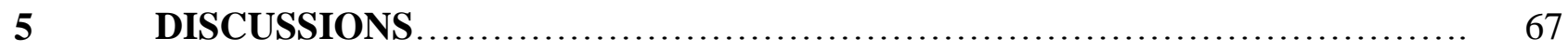

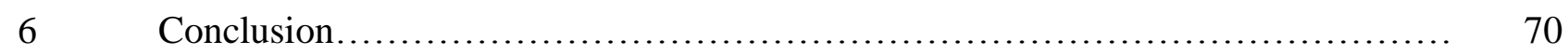

$6.1 \quad$ Future perspectives............................................... 70

\section{CHAPTER II - Exosomes (Nanovesicles)}

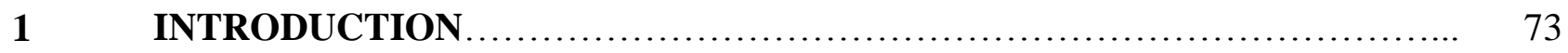

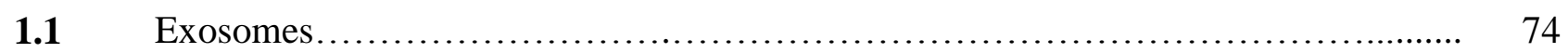

1.1.1 Biogenesis and secretion of exosomes................................. 75

1.1.2 Composition of exosomes and molecular contents........................ 77

1.1.2.1 Non-coding RNAs in exosomes.................................... 77

1.2 Isolation, purification and characterization of exosomes........................ 78

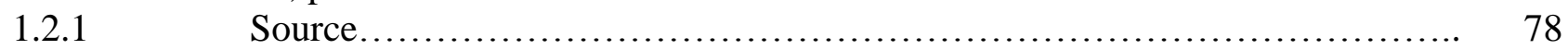

1.2.2 Technology for isolation............................................ 78

1.2.3 Technology for characterization...................................... 80

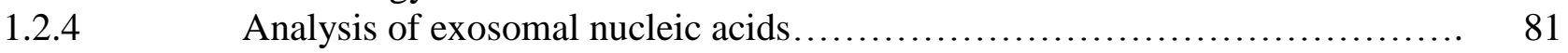

1.3 Biological functions of exosomes............................................ 82

1.3.1 Role in health, normal physiology and disease progression................... 83

1.4 Exosomes as mediators of cancer progression................................... 84

1.4.1 Exosome associated ncRNAs and cancer development...................... 85

1.5 Exosomes as diagnostic and prognostic biomarkers............................ 87

1.6 The therapeutic applications of exosomes..................................... 88

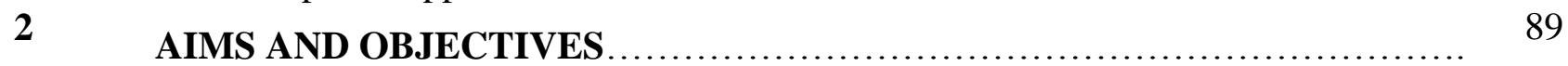

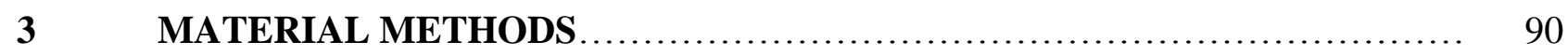

3.1 Preparations of exosomes from supernatant of cancer cell lines...................... 90

3.2 Isolation and purification of exosomes....................................... 90

3.3 Exosome estimation............................................................ 90

3.4 RNA isolation from exosomes ............................................... 91

3.5 Detection of exosomes by exosomal markers................................. 91

3.6 Identification of proteins from exosomes.................................... 91

3.7 Network analysis of proteins identified in breast and lung cancer exosomes........... 91

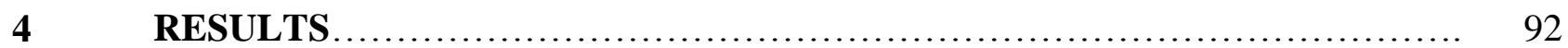

4.1 Detection of exosomal markers........................................... 92

4.2 Detection of RNA in exosomes and comparison with parent cells.................. 93

4.3 Comparison of total RNA and proteins from exosomes............................ 94

4.4 Identification of proteins in exosomes by LC-MS/MS ............................ 95

4.5 Network analysis of proteins in breast cancer-derived exosomes................... 98

4.6 Network analysis of proteins in lung cancer-derived exosomes...................... 99 
5 DISCUSSIONAS AND CONCLUDING REMARKS ....................... 100

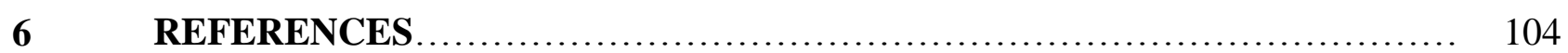

Electronic databases..................................................... 125

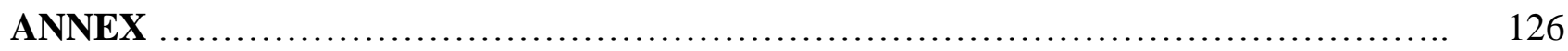

Annex A: Ethical committee approval certificate................................... 126

Annex B: List of publications in peer reviewed scientific journals..................... 127

Annex C: Abstracts published in proceedings of conferences.......................... 131 


\section{List of Abbreviations}

AOA: Anaplastic oligoastrocytomas

AOD: $\quad$ Anaplastic oligodendrogliomas

ATRX: $\quad$ Alpha Thalassemia/Mental Retardation Syndrome X-Linked

CDKN2A: Cyclin Dependent Kinase Inhibitor 2A

CIC: Capicua transcriptional repressor

CNS: Central nervous system

DNA: Deoxyribonucleic acid

EGFR: $\quad$ Epidermal growth factor receptor

ENCODE: Encyclopedia of DNA Elements

EVs: $\quad$ Extracellular vesicles

FUBP1: $\quad$ Far Upstream Element Binding Protein 1

GBM: $\quad$ Glioblastoma

G-CIMP: $\quad$ CpG island methylator phenotype

GVD: Gene delivery vehicles

HOTAIR: HOX antisense intergenic RNA

IDH1: $\quad$ Isocitrate dehydrogenase - 1

IDH2: Isocitrate dehydrogenase - 2

ISEV: $\quad$ International Society for Extracellular Vesicles

LGGs: Lower-grade gliomas

lincRNA: Long intergenic ncRNA

IncRNAs: Long non-coding RNAs

MDR: $\quad$ Minimal deleted region

MGMT: Methylguanine Methyltransferase

miRNA: microRNA

MVBs: $\quad$ Multivesicular bodies

MVs: Microvesicles 
ncRNAs: Non-coding RNAs, non-protein coding RNAs

NGS: $\quad$ Next generation sequencing

nnWM: Non-neoplastic white matter

NOTCH1: Neurogenic locus notch homolog protein 1

OD: $\quad$ Oligodendrogliomas

OLIG1/2: Oligodendrocyte transcription factor $1 / 2$

PCV: $\quad$ Procarbazine, Lomustine, and Vincristine chemotherapy

PDGFR: Platelet growth factor receptor

PDLG: $\quad$ Primary diffuse leptomeningeal gliomatosis

piRNA: $\quad$ Piwi-interacting RNA

PLO: $\quad$ primary leptomeningeal ODs

PTEN: Phosphatase and tensin homolog

RISC: $\quad$ RNA-induced silencing complex

RNA: Ribonucleic acid

siRNA: $\quad$ Small interference RNA,

snoRNA: Small nucleolar RNA

snRNA: $\quad$ Small nuclear RNA

TCGA: $\quad$ The Cancer Genome Atlas

TERRA: Telomeric repeat containing RNA

TERT: Telomerase reverse transcriptase

TP53: $\quad$ Tumour suppressor phosphoprotein p53

UCGs: Ultraconserved genes

UCRs: Ultraconserved sequences

VEGF: Vascular endothelial growth factor

V-RNA: $\quad$ Vault RNA

WHO: World Health Organization 


\section{List of Figures}

Numbers

Titles

Figure 1. Molecular signatures and IDH wild-type versus IDH-mutated pathways in lower grade diffuse gliomas

Figure 2. Molecular pathways and common genetic alterations in oligodendroglial tumours

Figure 3. IDH-mutant/G-CIMP positive gliomas and $1 \mathrm{p} / 19 \mathrm{q}$ status

Figure 4. Microsatellite loci representing minimal deleted region at $1 \mathrm{p}$ and $19 \mathrm{q}$....

Figure 5. $1 p / 19 q$ translocation and allelic loss

Figure 6. Biogenesis and processing of microRNA

Figure 7. Differentially expressed miRNAs between grade III and grade II oligodendrogliomas

Figure 8. Differentially expressed mRNAs between grade III and grade II oligodendrogliomas.

Figure 9. Heatmap showing differentially expressed miRNAs between 3 groups

Figure 10. Heatmap showing differentially expressed genes between 3 groups....

Figure 11. Bar graphs showing the mean miRNAs expression variation according the tumour grade.

Figure 12. Bar graphs showing the mean mRNAs expression variation according the tumour grade.

Figure 13. MiRNAs with relative reduction in expression in grade III oligodendrogliomas...

Figure 14. Primary culture of grade II oligodendrogliomas

Figure 15. Primary culture of grade III oligodendrogliomas.

Figure 16. Biogenesis of exosome-vesicles and other vesicles

Figure 17. Exosome-mediated transport of non-coding RNAs and tumour progression

Figure 18. Detection of exosomal markers.

Figure 19. Detection and comparison of exosomal-RNA with cellular RNA

Figure 20. Estimation and comparison of exosomal RNA and exosomal proteins with cellular RNA and protein

Figure 21. Scheme for identification of the total protein content from exosomes

Figure 22. Comparison of identified proteins in exosomes from two different cancer cell types.

Figure 23. Network analysis of proteins identified in breast cancer derived exosomes

Figure 24. Network analysis of proteins identified in lung cancer-derived exosomes 96

Page 


\section{List of Tables}

Table 1. WHO 2007 grading scheme of primary brain tumours....................... 3

Table 2. WHO 2016 grading scheme of primary brain tumours...................... 4

Table 3. Top differentially expressed miRNAs in grade II oligodendrogliomas .......... 42

Table 4. Top differentially expressed miRNAs in grade III oligodendrogliomas............... 43

Table 5. Top differentially expressed genes in grade II oligodendrogliomas ............. 44

Table 6. Top differentially expressed genes in grade III oligodendrogliomas ............ 45

Table 7. Functions of top expressed genes in grade II and III oligodendrogliomas......... 51

Table 8. Overexpressed miRNAs and downregulated mRNAs-targets in grade III 54

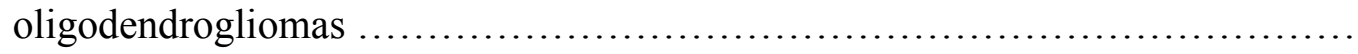

Table 9. Relative reduction of miRNAs expression in grade III oligodendrogliomas ...... 56

Table 10. Downregulated genes and overexpression of corresponding miRNAs in grade III 58 oligodendrogliomas

Table 11. Predicted biological roles of downregulated genes against upregulated miRNAs in grade III oligodendrogliomas

Table 12. Validated downregulated miRNAs and increased expression of their mRNA targets in grade III oligodendrogliomas

Table 13. Validated overexpressed miRNAs and decreased expression of their mRNA targets in grade III oligodendrogliomas 


\section{CHAPTER I}

Differential mRNA and miRNA expression in oligodendrogliomas of different grades of malignancy 


\section{ABSTRACT}

Oligodendroglial tumours originate from oligodendrocytes usually arising in the white matter and could be classified into grade-II oligodendrogliomas (OD) and anaplastic oligodendrogliomas (AOD, grade-III) according to the 2016 World Health Organization (WHO) grading scheme. ODs ${ }^{1}$ could be diagnosed by pathological and immunohistochemical analyses, however recent evidence suggests that they could be better diagnosed on the basis of defined genetic entities, such as the combined loss of chromosome 1p and 19q arms and IDH mutation. $1 \mathrm{p} / 19 \mathrm{q}$ co-deletion is molecular hallmark of ODs and is clinically associated with better prognosis, response to chemo/radio-therapy and overall survival. Typical oligodendroglial histological features are strongly associated with $1 \mathrm{p} / 19 \mathrm{q}$ loss and IDH mutation, which is critically important as diagnostic point of view.

The examining of exclusive molecular signatures and transcriptome expression profiles added to histological class could compliment the classification of OD subtypes. In this regard, microRNAs (miRNAs, miRs) profiles could serve classifier signatures for tumour subsets. MiRNAs are 22nt short non-coding RNAs which are expressed endogenously and regulate diverse cellular process through negative control on gene expression at the posttranscriptional level by direct or imperfect interaction with their target mRNAs. MiRNAs are involved in regulating human tumorigenesis acting as either tumour suppressors or oncogenes. During the passage of tumorigenesis miRNA expression level is significantly increased or decreased compared to corresponding normal tissue. The same is observed with their mRNAs. Therefore, transcriptome profiling of human tumours could identify signatures associated with progression, diagnosis, prognosis and response to therapy. However, until recently the information regarding the expression of miRNAs and mRNA in oligodendroglial tumours is scarce.

In this study we performed miRNA and mRNA differential expression profiling between grade II and grade III ODs using microarray based expression profiling platforms (723 transcripts and 41,000 genes, respectively). 7 cases for OD grade-II, and 7 for AOD grade-III, and 15 non neoplastic white matter (nnWM) samples were used after microdissection with no previous history of treatment. We performed a systematic evaluation of miRNAs and mRNAs expressions and determined miRNAs and putative target genes that are differentially expressed in grade III AOD, but not in grade II OD and in non-neoplastic white matter (nnWM).

\footnotetext{
${ }^{1}$ ODs when used with 's' will represent both OD and AOD.
} 
50 miRNAs were overexpressed and 43 were down regulated in AOD-III, whereas 7 miRNAs showed significant reduction in expressions in OD-II group. 3 miRNAs were commonly down regulated in comparisons of both groups. The hsa-miR-23a was strongly upregulated and hsamiR-27a was strongly downregulated in AOD-III. The functions of hsa-miR-23a and hsa-miR$27 \mathrm{a}$ were tested in human adult fibroblasts for cell proliferation assay and apoptosis detection. Cells treated with pre-miR-23a and pre-miR-27a showed $20 \%$ reduction in cell proliferation as compared with controls. Further, the functional relevance of miRNAs to their target mRNAs was validated for each group, using real time qPCR. 10 key-miRNAs from AOD were subjected to validation by qPCR. We were able to confirm 7 miRNAs ( $\leq 0.05)$. Among these, 5 miRs (miR193a-3p, miR-24, miR-27a, miR-30a-5p and miR-30c) showed reduced expression whose target genes (CCND1, HDAC2, PDGFA and RAB-26) were upregulated. Whereas, 2 miRNAs likewise miR-301b and miR-378 were overexpressed whose target genes BCL2, FGF2, CD44 and PPP4R4 confirmed by qPCR ( $\leq \leq 0.05)$. Bioinformatics based gene ontology (GO), and networking analysis revealed that differential expression and targets are attributed to differentiation of embryonic stem cells, cell adhesion, angiogenesis and neurogenesis, resistance to apoptosis, protein-protein interactions and cell proliferation.

It was possible to identify and validate miRNAs and their mRNA-targets potentially involved in the progression of oligodendrogliomas particularly in grade III-AOD. Collectively, this analysis provides new insights to malignant progression of oligodendroglial tumours and could compliment WHO-2016 diagnosis scheme and may provide predictive outcome in patients as well as decision to therapy.

\section{Keywords:}

Oligodendrogliomas, microRNA, mRNA, Transcriptome, Differential expression, Bioinformatics analysis, RT-PCR 


\section{RESUMO}

Oligodendrogliomas originários de oligodendrócitos que geralmente surgem na substância branca podendo ser classificados em grau oligodendroglioma (II-OD), e anaplastic oligodendrogliomas (grau III-AOD). Os ODs ${ }^{2}$ podem ser diagnosticados por análises patológicas e imuno-histoquímicas, porém evidências recentes sugerem que poderiam ser melhor diagnosticados com base em assinaturas moleculares, como a deleção combinada dos cromossomas 1p e 19q- marcadores moleculares dos OD associados clinicamente a um melhor prognóstico, resposta à terapia e melhor sobrevida. As características histológicas típicas dos oligodendrogliomas também estão fortemente associadas à deleção de $1 \mathrm{p} / 19 \mathrm{q}$, que é criticamente importante como ponto de vista diagnóstico. Assim, os subtipos de gliomas podem ser fortemente diferenciados não somente em relação ao seu perfil histológico mas também com base em seu perfil de expressão genica e suas assinaturas moleculares exclusivas. Os microRNAs (miRNAs, miRs) emergiram como assinaturas moleculares para os diferentes graus. Os miRNAs são RNAs não codificantes, contendo em torno de 22 nucleótidos. São expressos endogenamente e regulam diversos processos celulares através do controle negativo da expressão gênica em nivel pós-transcricional e por interacção directa ou imperfeita com o RNAm-alvo. Os miRNAs estão envolvidos na regulação da tumorigenese humana atuando como supressores de tumour ou oncogenes. Durante o processo da tumorigenese o nível de expressão dos miRNAs é aumentado ou diminuído significativamente em comparação com tecido normal correspondente. O perfil de expressão de miRNA de tumores humanos poderia identificar assinaturas associadas com progressão, diagnóstico, prognóstico e resposta à terapia. Contudo, até recentemente a informação sobre a expressão de miRNAs em oligodendrogliomas é escassa.

Neste estudo, avaliamos o perfil de expressão diferencial de miRNA e RNAm em ODs graus II e III usando plataformas de perfis de expressão baseadas em microarray (723 transcritos e 41.000 genes, respectivamente). Foram utilizados 14 casos de ODs microdissecados, sendo 7 OD grau II, e 7 AOD grau III (anaplasicos) sem histórico prévio de tratamento, além de 15 amostras de substancia branca não neoplásica (nnSB). Por meio de avaliações sistemáticas foram determinados miRNAs e mRNAs expressos em AOD grau III, mas não em OD grau II e em substancias brancas não neoplásicas (nnSB).

\footnotetext{
${ }^{2}$ ODs when used with 's' will represent both OD and AOD.
} 
Assim, foram encontrados 50 miRNAs com alta expressão e 43 miRNAs com baixa expressão em AOD-III, enquanto que 7 miRNAs apresentaram expressões reduzidas no grupo OD-II.

Na comparação entre os dois grupos, 3 miRNAs apresentaram baixa expressão. A hsa-miR-23a mostrou alta expressão e a hsa-miR-27a apresentou uma diminuição de expressão importante em AOD III. A atividade dos hsa-miR-23a e hsa-miR-27a foram testadas em células de fibroblastos adultos humanos usando ensaios de proliferação celular e detecção de apoptose. As células tratadas com pre-miR-23a e pre-miR-27a mostraram $20 \%$ redução de proliferação celular em comparação com os controles. Para cada grupo, a relevância funcional dos miRNAs e seus mRNAs alvos foi validada utilizando qPCR. Dos 10 miRNAs submetidos a validação em grau III, foi possivel confirmar 7 miRNA(p<0,05). Entre esses, 5 miRs (miR-193a-3p, miR-24, miR27a, miR-30a-5p e miR-30c) mostraram expressão reduzida, cujos genes alvos (CCND1, HDAC2, PDGFA e RAB-26) apresentavam alta expressão. Enquanto que, 2 miRNAs como miR-301b e miR-378 apresentaram alta expressão cujos genes alvo BCL2, FGF2, CD44 e PPP4R4 foram confirmados por qPCR $(\mathrm{p}<0,05)$. Ferramentas de bioinformática (Gene Ontology) e a análises em rede revelaram que a expressão diferencial e os alvos são atribuídos à diferenciação de células-tronco embrionárias, adesão de celular, angiogênese e neurogênese, resistência à apoptose, interações proteína-proteína e proliferação celular. Foi possível identificar e validar miRNAs e RNAm-alvos potencialmente envolvidos na progressão de oligodendrogliomas. Coletivamente, esta análise fornece novos achados relacionados a progressão maligna de tumores oligodendrogliais e poderia facilitar o diagnóstico preciso e mais restritivo, o desfecho preditivo em pacientes, bem como auxiliar na decisão da terapia.

\section{Palavras-Chave:}

Oligodendrogliomas, microRNA, RNAm, Expressão diferencial, Análise bioinformática, RTPCR 


\section{INTRODUCTION}

\subsection{Primary brain tumours}

Primary brain tumours are a heterogeneous group of lethal cancers and comprise a wide range of histological and pathological entities, each with a distinct natural history [1-3]. Primary cerebral tumours account for approximately $2 \%$ of all cancers. Although less incidence rate than leukaemia, the brain tumours have surpassed leukaemia as leading cause of cancer death, due to availability of intense therapeutic treatment to leukaemia in recent two decades [4]. According to 2016 cancer statistics for tumours of the brain and other nervous system approximately 23,770 estimated new cases including men and women are diagnosed and $\sim 16,050$ deaths occur per year in the United States [4]. This increasing incidence with new cases of primary brain tumours and other nervous system cancers is estimated $\sim 6.4$ per 100,000 per year and annual number of deaths is $\sim 4.3$ per 100,000 including men and women, according to NIH-National Cancer Institute, SEER Cancer Statistics Review, 1975-2013 report released on 2016 (http://seer.cancer.gov/csr/1975_2013/). These statistics are age-adjusted and are based on 20092013 cases and deaths.

For simplicity, central nervous system (CNS) tumours are classified as gliomas or non-gliomas [5]. Gliomas (neuroglial tumours) are the most common primary brain tumours emerging in adult human central nervous system and include a variety of histologic types and morphologies $[3,5]$. The diversity of glioma correlates with their different cells of origin and a wide spectrum of mutations and cell signalling pathways [6]. Histologic evaluation remains the gold standard for glioma diagnosis; however, diagnostic difficulty may arise from tumour heterogeneity, overlapping morphologic features, and tumour sampling [5]. For this reason genetic signatures are being considered potential marks to discriminate histologic and morphologic overlapping [3, 7]. 


\subsubsection{Classification of primary brain tumours}

Gliomas include differentiated astrocytic, oligodendroglial and ependymal tumours which account for more than $70 \%$ of all CNS tumours [8]. Gliomas have been traditionally classified according to their histopathology and the cell-type markers they express. According to World Health Organization (WHO) 2007 classification scheme the gliomas were divided into astrocytomas, oligodendrogliomas (ODs) and mixed gliomas (oligoastrocytomas) [2]. Astrocytomas correspond to most prevalent group of CNS tumours, and are subdivided into WHO grade-I to grade IV according to cellularity, atypia, presence of mitoses, microvascular proliferation and/or necrosis [2,9]. Grade I includes pilocytic astrocytoma, grade II corresponds to diffuse astrocytoma, grade III includes anaplastic astrocytoma and grade IV includes glioblastoma (GBM). This classification scheme has been updated in the 2016 WHO version which constitutes the edition of newly recognized neoplasms, and has deleted some entities, variants and patterns that no longer have diagnostic and/or biological relevance and have made major restricting criteria by incorporating genetically defined entities [3]. Among the high-grade astrocytomas, GBMs are the most frequent (65\%), most malignant of all adult gliomas and are one of the most lethal tumours in humans $[2,10,11]$.

Oligodendroglial tumours $(\mathrm{ODs})^{\mathbf{3}}$ comprise oligodendrogliomas (OD, i.e. grade II) and anaplastic oligodendrogliomas (AOD, i.e. grade III), whereas oligoastrocytic tumours (also known as mixed gliomas) comprise oligoastrocytomas and anaplastic oligoastrocytomas (grade II and grade III) (Table 1 and Table 2). The WHO 2007 classification system was based on histopathological features corresponding to different grades. Hitherto, WHO-2007 histopathologic classification system is well established, nevertheless, it presents high intra- and inter-observer variability, particularly among grade II-III tumours [12, 13]. According to the WHO 2016 classification system of CNS tumours, the molecular parameters should be used in addition to histology for defining many tumour entities. This updated classification system is both a conceptual and practical advance over its 2007 predecessor and formulates an advanced concept for how CNS tumour diagnoses should be structured in the molecular era [3]. The WHO 2016 CNS grading is summarized in Table 2, however here only the relevant classes (i.e. oligodendroglial tumours and related diffuse lesions), that we used in our study are mentioned.

\footnotetext{
${ }^{3}$ ODs when used with 's' will represent both OD and AOD.
} 
Table 1 : WHO 2007 grading system of primary brain tumours

Only major classes with relevance to our study are presented here. Note that this grading system did not use genetically defined entities.

\begin{tabular}{|l|l|l|l|l|}
\hline Tumour type & \multicolumn{3}{l}{ Grade } \\
\hline Astrocytic tumours & I & & & \\
\hline Pilocytic astrocytoma & & II & & \\
\hline Diffuse astrocytoma & & & III & IV \\
\hline Anaplastic astrocytoma & & & & \\
\hline Glioblastoma (GBM) & & & \\
\hline Oligodendroglial tumours (ODs) & II & & \\
\hline Oligodendrogliomas (OD) & & III & \\
\hline Anaplastic oligodendrogliomas (AOD) & & & \\
\hline Oligoastrocytic tumours (mixed gliomas) & & II & & \\
\hline Oligoastrocytoma & & & III & \\
\hline Anaplastic oligoastrocytoma & & & \\
\hline Ependymal tumours (ependymomas) & I & & & \\
\hline Subependymoma & & & III & \\
\hline Ependymoma & & & \\
\hline Anaplastic ependymoma & & & \\
\hline
\end{tabular}


Table 2: WHO 2016 grading system of primary brain tumours with added genetically defined entities

Only major classes with relevance to our study are presented here. Note that this grading system uses the genetically defined entities.

\begin{tabular}{|c|c|c|c|c|}
\hline Tumours with genetic entities & \multicolumn{4}{|c|}{ Grade } \\
\hline \multicolumn{5}{|l|}{ Astrocytic tumours } \\
\hline Pilocytic astrocytoma & $\mathrm{I}$ & & & \\
\hline Subependymal giant cell astrocytoma & $\mathrm{I}$ & & & \\
\hline Pleomorphic xanthoastrocytoma & & II & & \\
\hline Anaplastic pleomorphic xanthoastrocytoma & & & III & \\
\hline \multicolumn{5}{|l|}{ Diffuse astrocytic tumours } \\
\hline Diffuse astrocytoma, IDH-mutant & & II & & \\
\hline Anaplastic astrocytoma, IDH-mutant & & & III & \\
\hline Glioblastoma (GBM) IDH-wild type & & & & IV \\
\hline Glioblastoma (GBM) IDH-mutant & & & & IV \\
\hline Diffuse midline glioma, H3K27M-mutant & & & & IV \\
\hline \multicolumn{5}{|l|}{ Oligodendroglial tumours (ODs) } \\
\hline $\begin{array}{l}\text { Oligodendroglioma, } \\
\text { IDH-mutant and } 1 \mathrm{p} / 19 \mathrm{q} \text {-codeletion }\end{array}$ & & II & & \\
\hline $\begin{array}{l}\text { Anaplastic oligodendrogliomas, } \\
\text { IDH-mutant and } 1 \mathrm{p} / 19 \mathrm{q} \text {-codeletion }\end{array}$ & & & III & \\
\hline \multicolumn{5}{|l|}{ Ependymal tumours (ependymomas) } \\
\hline Subependymoma & I & & & \\
\hline Myxopapillary ependymoma & $\mathrm{I}$ & & & \\
\hline Ependymoma & & II & & \\
\hline Ependymoma, RELA fusion-positive & & II or III & & \\
\hline Anaplastic ependymoma & & & III & \\
\hline
\end{tabular}




\subsubsection{Diffuse lower grade gliomas}

Most critical type of gliomas are diffuse gliomas largely due the fact that they diffusely infiltrate the surrounding brain tissue and thus appear to be resistant to surgical resection. Diffuse lowergrade gliomas (LGGs) usually exhibit relatively slow growth and are infiltrative brain tumours which include WHO grade II astrocytomas, oligodendrogliomas and oligoastrocytoma. In contrast high-grade gliomas (HGGs) are represented by rapid growth and comprise WHO grades III and IV [5]. LGGs almost always progress, typically to GBM (grade IV), and are considered uniformly fatal. In diffuse gliomas active invasion occurs by neoplastic cells, of the adjacent parenchyma up to 3-4 cm from the "margin" of tumour resection. This makes it impossible to complete the neoplasia excision or surgical resection. Therefore, discrimination between LGGs of astrocytic and oligodendroglial origin remains an impeding challenge in neuro-oncology.

There is emerging focus in classifying and delineating glioma subtypes based on their exclusive molecular signatures than histological class $[14,15]$. The incorporation of clinically relevant genetic alterations (molecular signatures) is now being considered a part of the diagnosis of CNS tumours, given the fact that the molecular parameters added to histological analysis potentially redefines many tumour entities [3]. The availability of the datasets generated by The Cancer Genome Atlas (TCGA) serve as a potential platform for molecular characterization of distinct gliomas subsets including diffuse ones [7, 13, 16, 17].

It is now evident that diffuse gliomas are characterized by mutually exclusive TERT and ATRX mutations $[16,18,19]$. Surprisingly, 1p/19q co-deleted tumours have the same abnormality as GBM (TERT mutations) whereas diffuse astrocytomas regardless of grade, are characterized by $A T R X$ mutations. A targeted next generation sequencing (NGS) for mutations in ATRX, TERT, TP53, IDH1, IDH2, CIC, FUBP1, PI3KC, EGFR, H3F3A, BRAF, PTEN, and NOTCH genes as well as copy number alterations of chromosomes 1p, 19q, 10q and 7 has allowed a clinically relevant classification of diffuse anaplastic oligodendrogliomas (AOD) into groups with very different outcomes [20]. Mellai et al, has proposed that adult gliomas including oligoastrocytomas could be re-classified based on ATRX, IDH1/2 and 1p/19q status [21].

The integrative analysis of isocitrate dehydrogenase-1/2 (IDH1/2) wild-type (wt) LGGs with methylation and genetic profiles exhibited the features of "pre-GBM" as they formed distinct 
hypomethylation and gene expression clusters, mostly were grade III astrocytomas but their molecular alterations such as EGFR amplifications, PTEN mutations, CDKN2A loss and RTK gene fusions were typical of GBM and had short survivals. In contrast IDH1/2 mutants LGGs were hypermethylated. One subgroup was enriched for ODs with $1 \mathrm{p} / 19 \mathrm{q}$ co-deletion, CIC and FUBP mutations. Another subset was enriched for astrocytomas with TP53 and ATRX mutations. Interestingly, these groups clustered separately on gene expression analysis. A fourth gene expression cluster had elements of these two IDH1/2 mutant subgroups and the longest survival. A cluster-of-cluster analysis of mRNA, copy number, microRNA (miRNA) and methylation analyses reinforced the clear separation of three molecular classes of LGG based on the status of $I D H 1 / 2$ and 1p/19q [22]. These data illustrate a potential stratification of LGGs that emphasizes molecular characteristics. IDH wt LGG have molecular alterations and clinical behaviour similar to GBMs, whereas IDH mutant LGGs segregate based on 1p/19q status [22].

Such patterns at large were recently reported from TCGA based comprehensive study providing several potential insights into molecular signatures of diffuse gliomas. A genomewide comprehensive analysis of diffuse LGGs from large cohort of adult patients was performed for exome sequence, DNA copy number variations, DNA methylation patterns, mRNA and miRNA expression patterns, and targeted protein expression [7]. The integrated analysis of these patterns was made and examined for correlation with clinical outcomes. The integration of genomewide data from multiple platforms (methylation patterns, mutations, sequencing, and expressions) revealed three distinct molecular classes of LGGs those were non-overlapping and prognostically significant. These subclasses were delineated more accurately and concordantly by $I D H, 1 \mathrm{p} / 19 \mathrm{q}$, and TP53 status than histologic class. Nearly all LLGs with an IDH mutation either had 1p/19q co-deletion or carried a TP53 mutation (94\%) and ATRX inactivation (86\%). Strikingly, the large majority of LLGs without an IDH mutation were molecularly and clinically similar to GBM. Importantly, the LLGs patients with $I D H$ mutations and $1 \mathrm{p} / 19 \mathrm{q}$ co-deletions had the most favourable clinical outcomes. Moreover, their gliomas harboured mutations in $C I C, F U B P 1$, NOTCH1, and the TERT promoter [7], which are very consistent with as proposed by Gonda et al., [22].

TCGA based analysis of such molecular features suggests that incorporation of these molecular platforms and orthogonal intersection of molecular characteristics with existing genetic markers, 
such as IDH1 mutation, TP53 mutation, and 1p19q status, should facilitate accurate and reliable pathological diagnosis of diffuse gliomas [7, 22]. Moreover, the retrospective analysis of sequencing results by next-generation platforms may improve the diagnosis/classification in gliomas [23]. In short, the classification of diffuse gliomas could be improved based on defined genetic entities that is in compliance with WHO-2016 system which emphasise that the incorporation of clinically relevant genetic parameters added to histopathological analysis may redefine many tumour entities [3]. This is concordant with scheme by Huse et al., 2014, emphasising that despite similar histopathology, the molecular (genetic and epigenetics) signatures are non-overlapping between diffuse gliomas that could clearly differentiate biologically distinct forms of glioma subtypes (Figure 1) [24]. This has been supported by recent study that epigenetic signatures may further compliment the diagnosis of diffuse gliomas [25]. In pertinent to diffuse gliomas, a recent study by Paul and colleagues proposes that the identification of methylation signatures could potentially complement WHO-2016 classification scheme of different subtypes of diffuse glioma [26]. The methylation status is also true for oligodendrogliomas that will be discussed in later sections (and figure 3). 


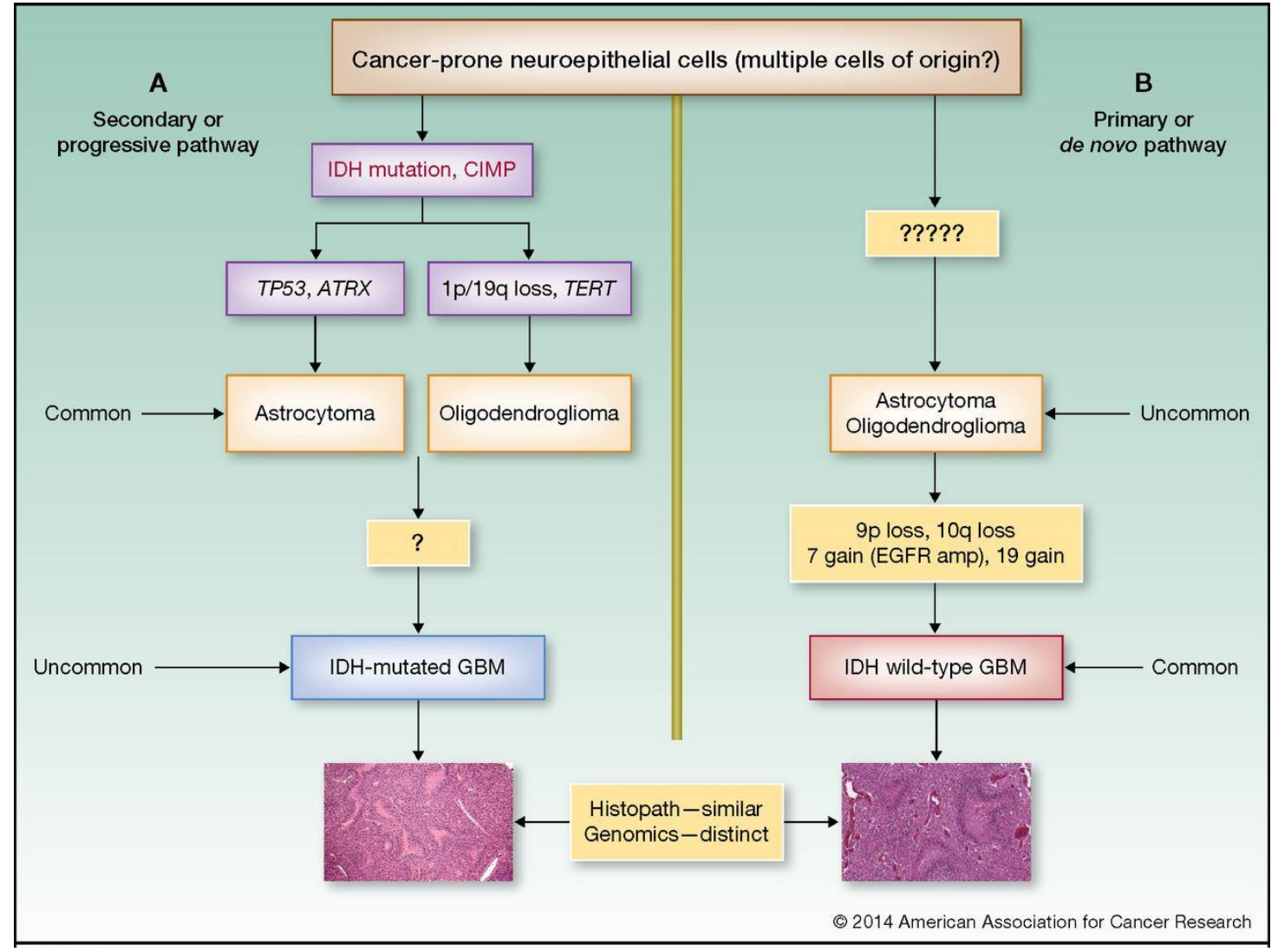

Figure 1. Molecular signatures and IDH wild-type versus IDH-mutated pathways in gliomas: Despite similar histopathology, the molecular (genetic and epigenetic) signatures are non-overlapping between diffuse gliomas which distinguish biologically distinct forms of glioma subtypes. (Adopted from Huse and Aldape 2014) [24]. 


\subsection{Oligodendrogliomas}

Oligodendrogliomas (ODs) ${ }^{4}$ are the glial tumours generally originate from oligodendrocytes in the brain parenchyma and usually arise in the white matter of the cerebral hemispheres. The oligodendroglial lesions are mainly found in the brain hemispheres with a predisposition for white matter and cortical infiltration. ODs are slow growing and rare primary brain tumours with cells that resemble oligodendrocytes. They were first described as a specific histologic type of glial neoplasm which share morphological similarities with mature oligodendrocytes [2, 27].

Generally, ODs arise as a solid mass but can also appear as a diffuse leptomeningeal lesion, oligodendrogliosis or primary diffuse leptomeningeal gliomatosis (PDLG) [28]. The PDLG is associated with rapid disease progression and mortality. The majority of these neoplasms are astrocytic, often high grade and the diagnosis is usually made postmortem [29, 30]. In contrast to primary leptomeningeal astrocytomas, the primary leptomeningeal ODs (PLO) are rare ODs [3136]. The presence of ODs in the ciliary indicates that gliomas can originate wherever neural stem cells exist and are difficult to diagnose [37]. Diagnosis can be made only after results of pathological and immunohistochemical analyses are known. Perry and colleagues reported a new form of neuronal astrocytic differentiation in oligodendroglial neoplasms and this pattern was referred as "ganglioglioma like maturation" given the combined morphologic and immunohistochemical evidence of ganglion cell differentiation and the loss of proliferative activity in comparison to adjacent oligodendroglial components [38].

In fact, grade II OD are well differentiated, slow growing tumours which can evolve into high grade anaplastic OD (AOD, grade III). Histologically/morphologically, the grade II OD are composed of uniformly round to oval cells with bland nuclear chromatin and often demonstrate perineuronal satellitosis, microcalcifications, mucoid/microcystic degeneration, and dense capillary networks. Subpopulations of cells may exhibit nuclear atypia and occasional mitoses. In contrast, the grade III AOD shows increased cellularity, cytological atypia and high mitotic activity. AOD often demonstrate vascular proliferation and necrosis as well.

According to WHO-2007 system, the ODs that contain both neoplastic oligodendrocytic and astrocytic cells were designated as mixed gliomas or oligoastrocytomas (Table 1). The low grade

\footnotetext{
${ }^{4}$ ODs when used with 's' will represent both OD and AOD.
} 
diffuse oligoastrocytomas (with astrocytic component, so called mixed gliomas) are histologically composed of well differentiated fibrillary or gemistocytic neoplastic astrocytes [27], and are histologically defined by the presence of oligodendroglial and astrocytic components. Moreover, OD and AOD have been thought difficult to establish clear differentiation from each other or even from other brain tumours [39]. However, according to WHO-2016 classification system these histotypes have been refined and complimented with genetic signatures (Table 2), which delineate mixed lesions based on genetic markers [3]. However, a recent report demonstrates imaging characteristics that are contradictory to WHO 2016 genetically defined oligodendrogliomas, concluding that these characteristics are indistinct and poorly circumscribed with WHO 2016 genetically defined oligodendrogliomas [40].

ODs are estimated to $20 \%$ of all glial tumours in adults [41], and have better prognosis than GBMs [4, 42, 43]. ODs predominantly occur in adults being more common in individuals with the age ranging from 4th to 5th decades, and are slightly more frequently in men than in women. The peak incidence is observed during the fifth decade (47 years is the median age at diagnosis), but can be seen in patients from 3 to 76 years of age. In a most recent survey, the trends in incidence rates and survival were calculated for OD and AOD by age, gender and race [44]. OD incidence peaked at 36-40 years, whereas AOD incidence peaked at 56-60 years. According to this survey, the survival rates decreased with advancing age for OD, whereas the cases aged between 0 to 24 years had the lowest survival for AOD. However, AOD proportion is increased among oligodendroglial tumours up to age 75. It is proposed that low AOD survival in 024 years may indicate molecular differences in paediatric cases; therefore, the surveillance of tumour-specific trends by age, race and gender can reveal clinically relevant variations. Collectively, this study reports a decrease in overall OD and AOD incidence from 2000 to 2013 [44].

\subsubsection{Molecular biology of oligodendrogliomas}

The ODs have clearly distinct molecular profiles [7, 13-15]. The molecular heterogeneity among tumours of the same type and grade could present variations in prediction of survival and response to therapy. Although the ODs are rare but interesting due to better prognosis than other tumours of this type, unique chemosensitivity and the durability of some of the responses due to 
unique molecular biology and presence of certain tumour genetic anomalies [45-49]. ODs were the first gliomas for which specific molecular predictors of chemotherapeutic response and overall survival were identified [50]. Reported molecular alterations in ODs differ greatly from those in astrocytomas, accounting for their distinct molecular signatures and clinical behaviour $[7,13]$.

Differential expression analyses of genes in the histopathological subsets may indicate the relationships between gene expression pattern and histological subtype and/or response to chemotherapy [51, 52], as well as extent of neoplastic infiltration [53]. Some highly expressed key genes in ODs are human $O L I G$ genes [54-56]. High levels of $O L I G$ expression are a general characteristic of oligodendroglial tumours while OLIG1 expression is specific for oligodendrocytes $[54,55]$. Thus $O L I G$ markers have a diagnostic potential to identify oligodendroglial tumours. Other key featured markers of glioma are oligodendrocyte progenitor cell marker NG2 with high tumour initiating potential in glioma cells [57, 58], while differentiation defective NG2+ cells are predominant cell type in human low-grade gliomas [59].

Oligodendroglial tumours also show $C A I X$ expression induced by hypoxia in tissues and plays a major role in the pathophysiology of oligodendroglial malignancies [60]. The $C A I X$ expression affects the survival of cancer cells as well as correlating with patient survival and may be a good target molecule to design new anticancer therapies [60]. Another important player is galectin1 (Gal-1) which plays important biological roles in oligodendroglial and astroglial cancer cells [6163]. Gal-1 has a key role in astroglioma and ODs cell migration [62, 64], as well as ODs neoangiogenesis and chemoresistance [65].

Overexpression of growth factors could initiate a proliferative signal in ODs and includes epidermal growth factor receptor (EGFR), vascular endothelial growth factor (VEGF) and platelet derived growth factor receptor (PDGFR) [20, 22, 55, 66-68]. EGFR overexpression is found in majority of ODs whereas amplification of PDGFR $\alpha$ is less in ODs [58] [55]. Majority of astrocytoma tumours exhibit amplification of PDGFR $\alpha$ and/or PDGFR $\beta$ genes, as well as the genes encoding their ligands such as PDGF-A and -B or -C and -D [67, 69-71]. 
Several other molecular mechanisms with distinctive genetic patterns have been described for ODs (Figure 2). However, this sequential model might be updated with emerging models in which two muationally different clones interact (for example may provide different secreted growth factors into microenvironment) to provide common proliferative advantage for progression for both clones. Although ODs tumour types share similar developmental hierarchies and lineages of glial differentiation, however differences in bulk profiles between IDH-mutant astrocytoma and OD can be primarily explained by distinct tumour microenvironment and signature genetic events. In light with these arguments, decoupling genetic influences from lineages, and microenvironment in IDH-mutant gliomas may help improve this sequential model. These arguments may also apply to model in Figure 1 (where there could be more than one cell of origin (clones) in the context of brain tumour onset and genetic influence).

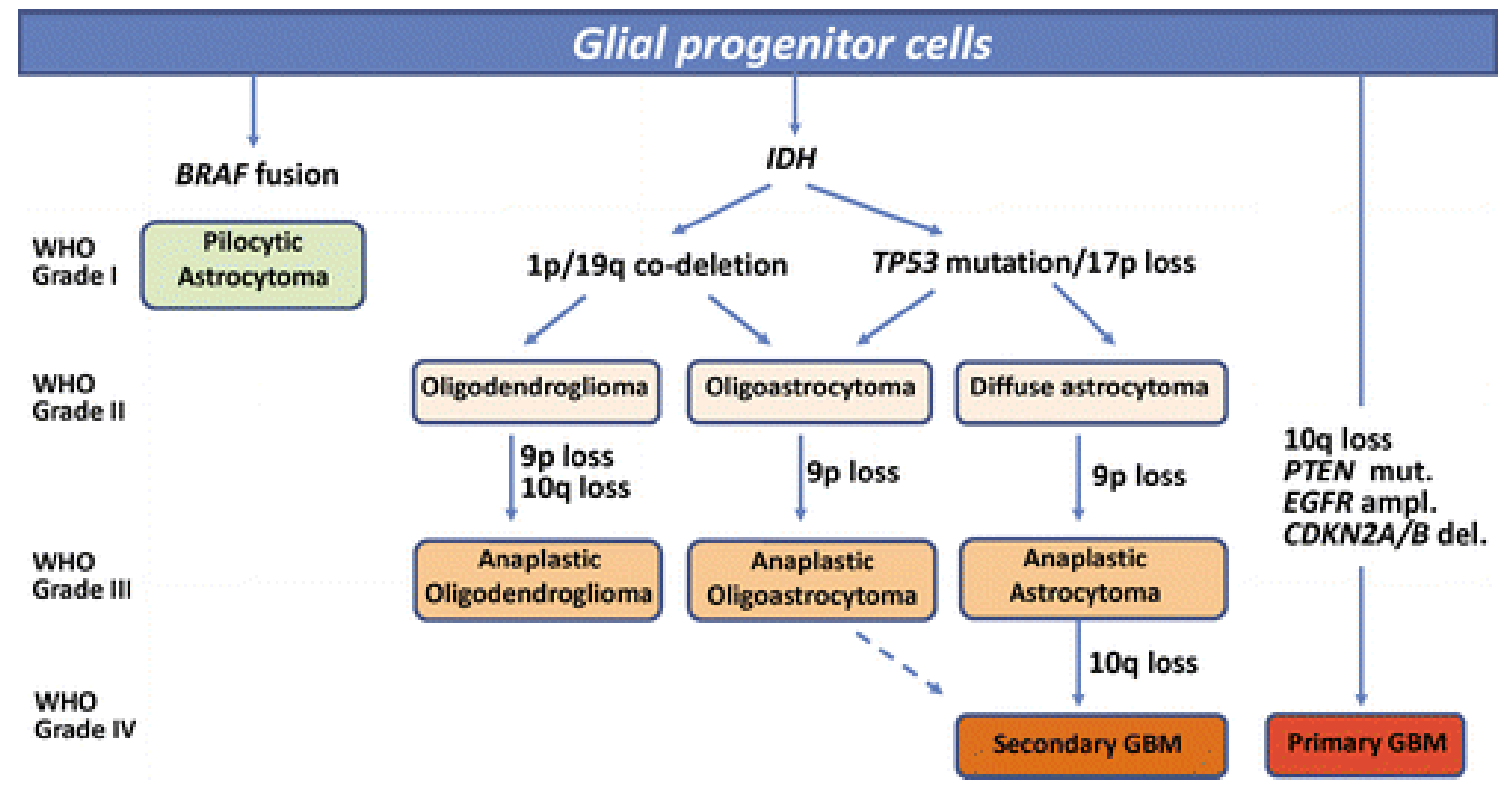

Figure 2. Molecular pathways and common genetic alterations in astrocytic, oligodendroglial and oligoastrocytic neoplasms (Adopted from Nikiforova and Hamilton 2011) [5]. 


\subsubsection{IDH1/2 and TP53 mutations}

Huge body of evidence has clarified that the majority of oligodendrogliomas, low grade diffuse astrocytomas and oligoastrocytomas show IDH1/2 gene mutation and mutation in TP53 [7, 72, 73]). IDHI and IDHI genes encode isocitrate dehydrogenase 1 and 2 respectively, and participates in the citric acid cycle. Mutations in $I D H$ induce chromatin remodelling and promoter hypermethylation which occurs in the promoter region of tumour suppressor genes [7, 13, 22, 74]. The IDH1 mutations are very early genetic events [5], whereas the acquisition of TP53 mutations may lead to astrocytic differentiation and subsequent loss of $1 \mathrm{p} / 19 \mathrm{q}$ lead to oligodendroglial phenotype.

Hitherto, IDHI mutations are frequent in diffuse astrocytic and oligodendroglial tumours, nonetheless are found as rare cases in other gliomas such as pilocytic astrocytomas and are apparently absent in ependymomas. WHO-2016 scheme considers the incorporation of genetic criteria of IDH-mutant and $1 \mathrm{p} / 19 \mathrm{q}$ co-deletion for strict designation of molecular signatures to oligodendroglial tumours. ODs with astrocytic component may additionally show mutation in TP53 gene. The TP53 gene encodes the cell cycle progression regulator Rb. Alterations in the $R b 1 / C D K 4 / p 16 I N K 4 A$ and $p 53 / p 14 A R F$ genes, lead to interruption of signals which result in deregulated cell proliferation [75]. 


\subsubsection{Oligodendrogliomas with mutations in TERT promoter $C I C$ and $F U B P 1$ genes}

The CIC gene located on chromosome 19q and FUBPI gene located on 1p respectively show somatic mutilations in ODs patients and contribute a critical role in the biology and pathology of human ODs [76]. More recently, recurrent molecular genetic alterations in ODs have been identified including mutations in $I D H 1 / 2, C I C, F U B P 1$, and the TERT promoter, as well as the absence of ATRX and TP53 alterations. These alterations occur concurrently with 1p/19qcodeletion, and definitively distinguish ODs from tumours [77]. This in important to emphasize that there does not seem to be a specific gene or even cytoband at $1 p$ or $19 q$ associated with $1 \mathrm{p} / 19 \mathrm{q}$-codeletion, therefore what exactly is going on with specific genes at this specific regions remains elusive. Recently it has been proposed that testing for TERT promoter mutations or ATRX alterations may compliment additional useful prognostic information in certain WHO2016 diagnostic groups [78].

Next-generation sequencing has identified novel mutations in $1 \mathrm{p} / 19 \mathrm{q}$ co-deleted tumours. CIC mutations are more frequent and occur more than $45 \%$ of ODs, whereas FUBP1 mutations occur $24 \%$ of ODs cases. However, these mutations are rare in astrocytomas or oligoastrocytomas accounting for $10 \%$ only [18]. It has been shown that ATRX mutations are associated with $I D H 1$ mutations and with an alternative lengthening of telomeres phenotype. $A T R X$ is frequently mutated in more than $70 \%$ cases of grade II-III astrocytomas, whereas ATRX mutations in oligoastrocytomas represent $68 \%$ of cases [18]. In another study, it was shown that CIC mutations are more frequent in ODs as compared to oligoastrocytomas, whereas CIC mutations in astrocytomas are rare [79]. Of particular note, $~ 100$ oligodendroglial tumours with CIC mutations were detected with $1 \mathrm{p} / 19 \mathrm{q}$ co-deletions. In contrast, CIC mutations in oligoastrocytomas were only presented with $\sim 50 \%$ of $1 \mathrm{p} / 19 \mathrm{q}$ co-deletion [79]. Although, FUBP1 mutations were detected in only $\sim 12 \%$ of tumours, but always was presented with CIC mutations.

Approximately $70 \%$ of ODs with 1p19q co-deletions harbour somatic mutations in the CIC gene on chromosome 19q13.2. Mutations in CIC and IDHI cooperatively regulate 2-hydroxyglutarate levels and cell clonogenicity in ODs cells in vitro [80]. A diffusely infiltrative xenograft model of human anaplastic ODs showed an infiltrative growth pattern, and harbouring mutations of 
IDH1 $(\mathrm{R} 132 \mathrm{H})$ and of the tumour suppressor genes, FUBPI and CIC. The xenografts were highly invasive, exhibiting a distinct migration and growth pattern around neurons. [81]. It is proposed that mutant $C I C$ on the single retained $19 \mathrm{q}$ allele is linked to the pathogenesis of oligodendrogliomas with IDH mutation [82]. Therefore, concurrent $C I C$ mutations, IDH mutations, and 1p/19q loss could distinguish ODs from other cancers.

More recently, recurrent molecular genetic alterations have been identified to occur concurrently with $1 \mathrm{p} / 19 \mathrm{q}$-codeletion, and definitively identify these tumours, including mutations in $I D H 1 / 2$, CIC, FUBP1, and the TERT promoter, as well as the absence of ATRX and TP53 alterations [77]. Recent molecular studies have shown that all 1p/19q co-deleted tumours have IDH mutations and most of them also have TERT mutations [83].

These findings provide a foundation for the consistent diagnosis of this tumour type, upon which a generation of clinical investigators have assembled a strong evidence base for the effective treatment of this disease with radiation and chemotherapy.

\subsubsection{Methylation of MGMT genes in oligodendrogliomas}

Large body of evidence clarifies that in addition to genetic mutations, the epigenetic changes also play an important role in ODs initiation and progression. A common finding in ODs is the methylation of DNA regions that code for Methylguanine Methyltransferase (MGMT) genes. $M G M T$ genes are responsible for DNA repair enzyme which is down regulated by hypermethylation. Methylation of MGMT genes is shown to be present in majority of ODs cases and contribute to higher chemosensitivity. Well documented example is of lipid phosphatase PTEN gene which maps to $10 \mathrm{q} 23$ [84]. Methylation of the promoter region results in down regulation of PTEN gene in oligodendroglial tumours [85].

Promoter hypermethylation represents a primary mechanism in the inactivation of tumour suppressor genes during tumorigenesis. $M G M T$ promoter hypermethylation is significantly more frequent and the percentage of methylated $\mathrm{CpG}$ sites in the investigated $M G M T$ promoter fragment is significantly higher in tumours with $1 \mathrm{p} / 19 \mathrm{q} \mathrm{LOH}$ as compared to tumours without allelic losses [86]. This suggests that MGMT hypermethylation and low expression of $M G M T$ genes are frequent in ODs and could contribute to the chemosensitivity of these tumours [86]. 
Hypermethylated p14ARF tumour suppressor genes have also been observed in ODs [87-89]. Approximately $50 \%$ of ODs show hypermethylation in promoter region or loss of the chromosome $9 \mathrm{p}$ region which results in downregulation or mutation of the tumour suppressor gene pl6INK4A/CDKN2A. It is now evident that the promoter hypermethylation of RB1 is frequent in ODs $[75,87,90]$. The INK4a/ARF locus on chromosome 9p21 encodes two gene products that are involved in cell cycle regulation through inhibition of CDK4-mediated RB phosphorylation (p16INK4a) and binding to MDM2 leading to p53 stabilization (p14ARF). The locus is deleted in up to $25 \%$ of ODs and $50 \%$ of anaplastic ODs. Aberrant p14ARF expression due to hypermethylation is the earliest INK4a/ARF change in the evolution of ODs, while the presence of p14ARF and p16INK4a deletions indicates progression to ODs [75, 87]. The aberrant frequency of $\mathrm{CpG}$ island hypermethylation methylation for several genes was more frequent in ODs and ependymomas. However, no methylation of these genes was detected in normal brain tissue samples. This aberration seems to occur early in the carcinogenesis process [89].

Oligodendroglial tumours frequently demonstrate hypermethylation of the CDKN2A (MTS1, p16INK4a), p14ARF, and CDKN2B (MTS2, p15INK4b) tumour suppressor genes [88]. hypermethylation of $C D K N 2 A, p 14 A R F$, and $C D K N 2 B$ is an important epigenetic mechanism by which oligodendroglial tumours may escape from p53- and pRb-dependent growth control [88]. RB1 CpG island hypermethylation is a common epigenetic event that is associated with the development of malignant nervous system tumours [91]. Concurrent promoter hypermethylations during ODs were detected in multiple genes including MGMT, RB1, p16INK4a, p73, p15INK4b and $p 14 A R F$ [90]. concordant hypermethylation of $p 16 I N K 4 a$ and $p 15 I N K 4 b$ were significantly associated with anaplastic ODs, and hypermethylation of MGMT was significantly associated with loss of chromosome $19 q$ and with combined loss of chromosomes 1p and 19q [90]. More importantly, several candidate tumour suppressor genes such as $p 16 I N K 4 a, p 15 I N K 4 b$, and $p 73$ that were previously reported as unmutated in ODs were found to be hypermethylated in their CpG islands [90].

Methylation of $\mathrm{CpG}$ island promoter has been proposed a novel mechanism of $C D K N 2 \mathrm{~A} / \mathrm{p} 16$ inactivation [92]. Recently methylation profiles in oligodendroglial tumours revealed more frequently methylated genes which include RASSF1A (80.3\%), CASP8 (70.5\%) and CDKN2A 
(52.5\%). Methylation of the ESRl promoter is significantly associated with shorter overall survival and progression-free survival, whereas the methylation of IGSF4 and RASSFIA is significantly associated with shorter duration of progression-free survival [93].

Since MGMT $\mathrm{CpG}$ island is invariably methylated in adult astrocytic and oligodendroglial tumours with $I D H 1$ or $I D H 2$ mutations, it was suggested that MGMT methylation may be one of the earliest events in the development of astrocytic and oligodendroglial tumours [94]. Methylation of the MGMT promoter, $1 \mathrm{p} / 19 \mathrm{q}$ co-deletion, mutated $I D H 1$, and $\mathrm{CN}-\mathrm{LOH}$ of $17 \mathrm{p}$ were the most frequent genetic aberrations in ODs. Key feature was the methylation of the MLH3 gene promoter in large majority of LGGs [95]. ODs with LOH at 1p-19q and IDH1 mutations also exhibit MGMT promoter methylation and GSTP1 promoter methylation with good prognosis [68]. There exists a correlation between MGMT promoter methylation status, $1 \mathrm{p} / 19 \mathrm{q}$ deletions, and response to temozolomide (TMZ) in anaplastic and recurrent oligodendrogliomas. Therefore, MGMT promoter methylation in randomized chemotherapy trials may help predicting patient survival [96]. MGMT promoter methylation is related to tumour progression, particularly in tumours with an intact $1 \mathrm{p}$ arm. These observations suggest that MGMT promoter methylation is a late event in progressive oligodendrogliomas, and therefore, their chemosensitivity is not necessarily related to MGMT methylation status [97].

Outcomes for patients with anaplastic astrocytoma, anaplastic oligoastrocytoma, and anaplastic oligodendrogliomas (AOD) treated with (RT) with and without TMZ were examined. This study proposes that there is a benefit to concurrent RT plus TMZ that is independent of adjuvant monthly TMZ [98]. It suggests that the use of RT with TMZ could benefit the postoperative management of grade III anaplastic gliomas. It has been shown that IDH mutations in patients are positively correlated with MGMT methylation [99]. MSH6 mutations were found to influence the sensitivity of tumour-initiating cell lines (BTICs) to TMZ both; in vitro and in vivo, independent of $M G M T$ promoter methylation status [100].

It is also equally clear that benefit to procarbazine, lomustine, and vincristine (PCV) chemotherapy is not limited to the $1 \mathrm{p} / 19 \mathrm{q}$ co-deleted cases; potential other predictive factors are IDH mutations and MGMT promoter methylation [83]. Recently whole exome sequencing was performed on short term and long term survivors who received PCV chemotherapy plus 
radiotherapy (RT), to examine if there were specific genetic alterations that distinguish ODs patients with $1 \mathrm{p} / 19 \mathrm{q}$ co-deletion who benefit from PCV from those who do not [101]. It was concluded that IDH, FUBP1 and CIC, mutations as well as rs55705857 genotype are common in ODs, and no distinct genetic signature was identified to differentiate short term and long term survivor. In another study it was reported that the patients who received adjuvant RT with concomitant chemotherapy ((chemoRT), (concomitant or sequential)) demonstrate significantly improved 5 year overall survival as compared to those who received adjuvant RT alone or no adjuvant therapy [102]. Multivariate analysis revealed that adjuvant chemoRT (concomitant or sequential) is independent prognostic factor for better overall survival in AOD and suggests that it should be considered for all clinically suitable patients who have undergone surgery [102, 103].

Oligodendroglial and astrocytic gliomas often show hypermethylation and aberrant expression of EMP3 gene at 19q13.3 which previously have been considered as tumour suppressor gene [104]. In contrast, overexpression of EMP3 gene is associated with retaining chromosome arms $1 \mathrm{p}$ and 19q in ODs, and does not support EMP3 as the target TSG on chromosome 19q13 in ODs [105]. Methylation of the EMP3 gene promoter together with $\mathrm{LOH} \mathrm{19q}$ serves as an indication for treatment with adjuvant therapy ab initio in order to improve the overall survival of OD-II patients [106]. In contrast, $N H E-1$ on $1 \mathrm{p}$ is silenced in ODs in relation to $I D H$-associated hypermethylation and $1 \mathrm{p}$ allelic loss [107].

ODs are more likely to exhibit the glioma-CpG island methylator phenotype (G-CIMP), relative to low-grade astrocytomas [22]. Hypermethylation of $\mathrm{CpG}$ islands is a common epigenetic event that is associated with the development of oligodendroglial tumours [90]. It is now clear that, in addition to genetic features of key-genes of gliomas, the methylation patterns, such as $\mathrm{CpG}$ island methylator phenotype (G-CIMP) could aid in molecular profiling of low grade lesions of astrocytic and oligodendroglial tumours [22, 108]. ODs and low-grade astrocytomas exhibit distinctly different G-CIMP status. For example, TCGA profiles of LGGs reveal that ODs are more likely to exhibit G-CIMP status, relative to low-grade astrocytomas [22]. Of particular note, the G-CIMP are tightly associated with IDH1 somatic mutations and are more prevalent in grade II and III diffuse gliomas and are within the proneural expression subgroup [24, 109] 
(Figure 3). These can be further sub-classified on the basis of $1 \mathrm{p} / 19 \mathrm{q}$ co-deletion (frequently showing ODs histology) or TP53 mutation (frequently showing astrocytic histology).

The G-CIMP-positive patients are younger at the time of diagnosis and exhibit significantly improved outcome [109], indicating G-CIMP as a distinct subset of low grade gliomas on molecular and clinical grounds. IDH mutation-associated G-CIMP profiles featured one group of ODs with $1 \mathrm{p} / 19 \mathrm{q}$ loss while other G-CIMP group with intact $1 \mathrm{p} / 19 \mathrm{q}$ and frequent TP53 mutation [108]. It is proposed that CIC-associated G-CIMP hypermethylation in oligoastrocytomas does not provide an alternative mechanism of functional CIC protein abrogation [110]. The strong association between CIMP status and MGMT promoter methylation suggests that the MGMT promoter methylation status is part of a more general, prognostically favourable genomewide methylation profile. Methylation profiling therefore may help identify (AOD) and anaplastic oligoastrocytomas (AOA) with improved prognosis [111]. Identification of epigenetic i.e. methylation signatures may further compliment the patient outcome $[25,26]$.

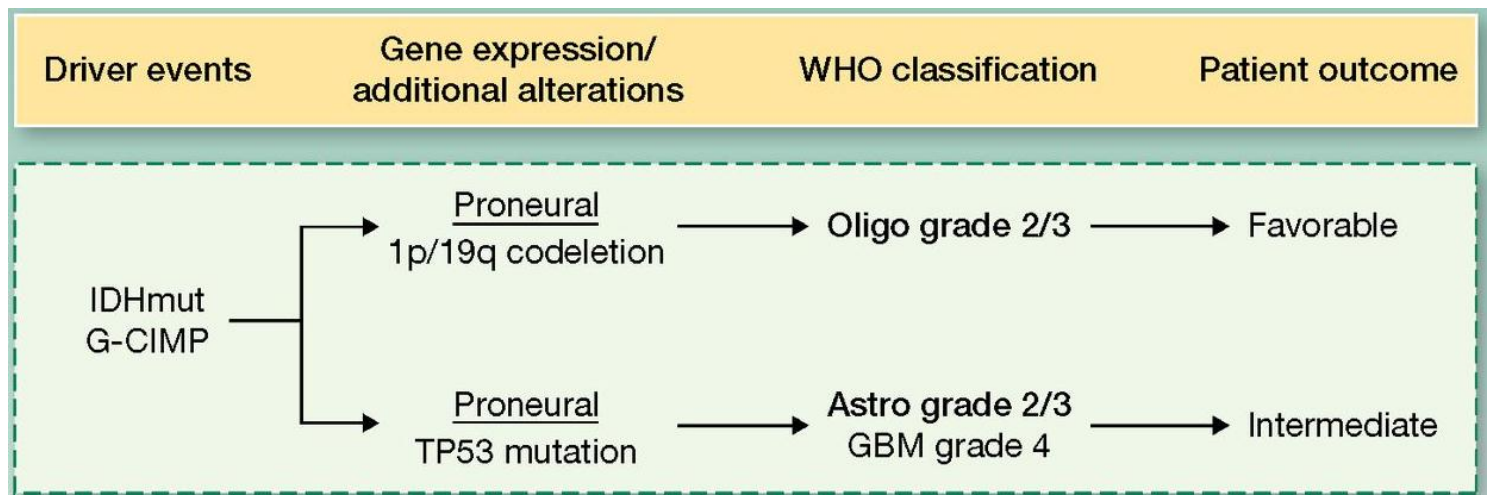

Figure 3. Large majority of grade II and III diffuse gliomas correspond to IDH-mutant/GCIMP-positive gliomas (top) and are within the proneural expression subclass. These can be 
further sub-classified on the basis of 1p/19q co-deletion (with ODs histology) or TP53 mutation (with astrocytic histology) [24].

\subsection{Genetics of 1p/19q co-deletion and loss of heterozygosity}

Among all described genetic alterations, the abnormalities in chromosomes $1 \mathrm{p}$ and $19 \mathrm{q}$ are the most significant. ODs and anaplastic ODs frequently exhibit loss of heterozygosity (LOH) on chromosomes 1p and 19q. This combined loss (so called co-deletion) of chromosome arms 1p and $19 \mathrm{q}$ is a hallmark of oligodendroglial tumours particularly in grade II ODs (Figure 4). This allelic loss at $1 \mathrm{p} / 19 \mathrm{q}$ is observed in $70 \%$ to $85 \%$ of ODs [112]. Reifenberger et al., in 1994 for the first time described combined loss of the entire short arm of chromosome 1 and the long arm of chromosome 19 (combined $1 \mathrm{p} / 19 \mathrm{q}$ loss) as the most characteristic genetic lesion in ODs [113]. The loss of genetic information from $19 q$ and $1 p$ as well as the rarity of TP53 mutations in oligodendroglial tumours suggests that the early events in their oncogenesis are distinct from those associated with astrocytic tumours [113]. However, similarities are indicated by the allelic losses on $9 p$ and $10 q$ in the anaplastic tumours, suggesting the utilization of common pathways of progression [113].

Subsequently, Mainz et al., showed that low-grade mixed oligoastrocytoma exhibit either characteristic astrocytic lesions (TP53 mutations) or combined loss of $1 \mathrm{p} / 19 \mathrm{q}$, pointing out that at the molecular level mixed tumours do not exist [114]. According to this study there exist genetically two distinct subsets of oligoastrocytomas; one genetically related to astrocytomas while other genetically being related to ODs. However, histologically, the oligoastrocytomas having TP53 mutations were more often astrocytoma-predominant, whereas those with 19q loss were more often ODs-predominant [114]. The alone or combined $1 \mathrm{p} / 19 \mathrm{q}$ deletion is detected in up to $80 \%$ grade II ODs and $60 \%$ of grade II anaplastic ODs. In astrocytic tumours, the deletion can be observed up to $10 \%$ including GBM and mixed tumours (oligoastrocytomas) up to $50 \%$ of cases, depending of series [115].

Based on these studies, two parallel discoveries made another important observation that the combined loss of $1 \mathrm{p} / 19 \mathrm{q}$ represents a balanced translocation, after which a copy of $1 \mathrm{p}$ and $19 \mathrm{q}$ is lost [116, 117] (Figure 5 A). Morphologically defined GBM with ODs component (GBMO) does not correspond to a particular genetic signature, but rather represents a collection of 
genetically dissimilar entities. For determining these molecular subtypes based on IDH and $1 p / 19 q$ status, the ancillary testing should be used [118]. Moreover, comparison of radiological imaging features may discriminate GBMO from conventional GBM (i.e. WHO grade IV) [119].

Another subset of ODs shows, homozygous deletion of the CDKN2A tumour suppressor gene located on 9p21 $[50,120]$. The $C D K N 2 A$ loss is associated with a significantly worse prognosis as compared to $1 \mathrm{p}$ and $19 \mathrm{q}[50]$. $1 \mathrm{p}$ loss is inversely related to deletions of the CDKN2A gene on 9p, which encodes the key cell cycle regulatory molecule p16INK4A. In recent study, point mutations of the CDKN2C gene mapped of chromosomal band $1 \mathrm{p} 32$ has been reported. However, these cases did not have $C D K N 2 A$ gene deletions [121]. A mild increase in perfusion and metabolism has been observed in $1 \mathrm{p} / 19 \mathrm{q}$ co-deleted ODs than $1 \mathrm{p} / 19 \mathrm{q}$ intact ODs [122].

\section{Chromosome 1 Chromosome 19}

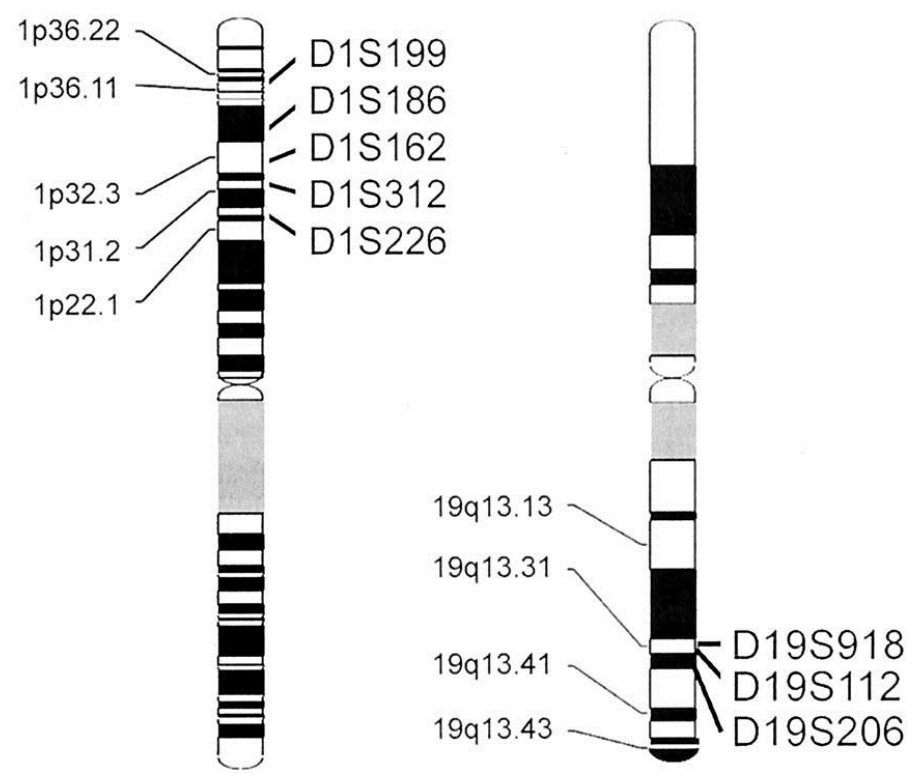

Figure 4: Microsatellite loci showing shortest common regions corresponding to minimal deleted region (MDR) of 1p.21 and 19q.13 loss of heterozygosity (LOH). Adopted from Hatanpaa et al., 2003 [123]. 


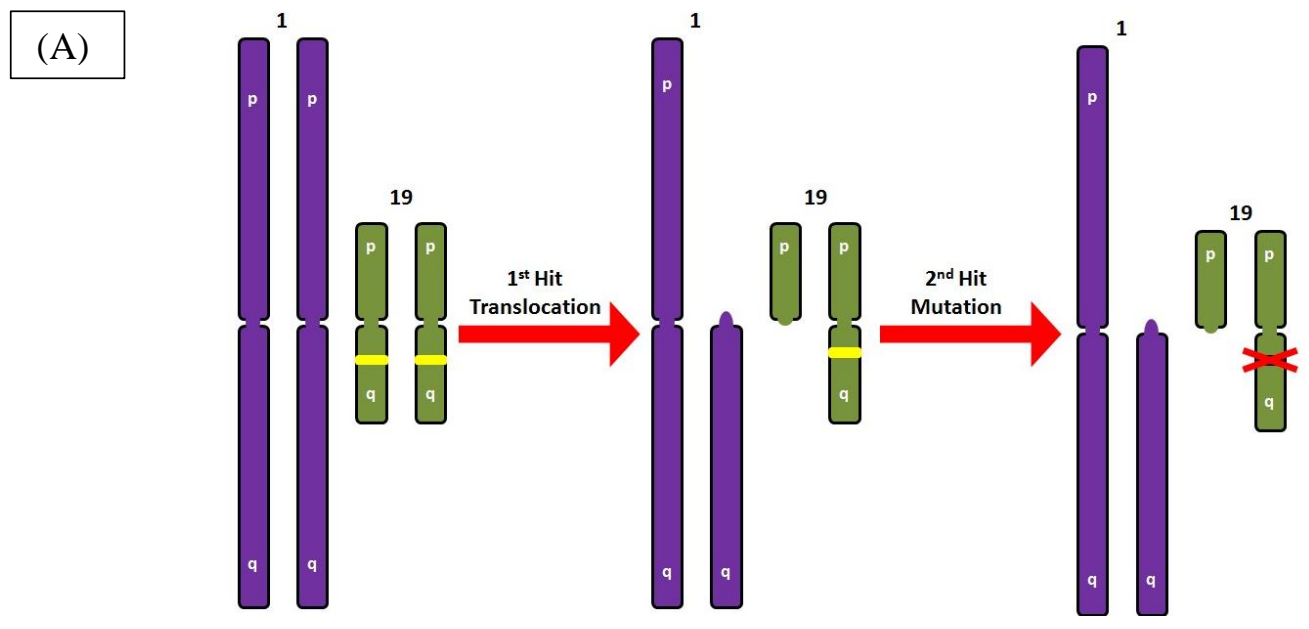

(B)

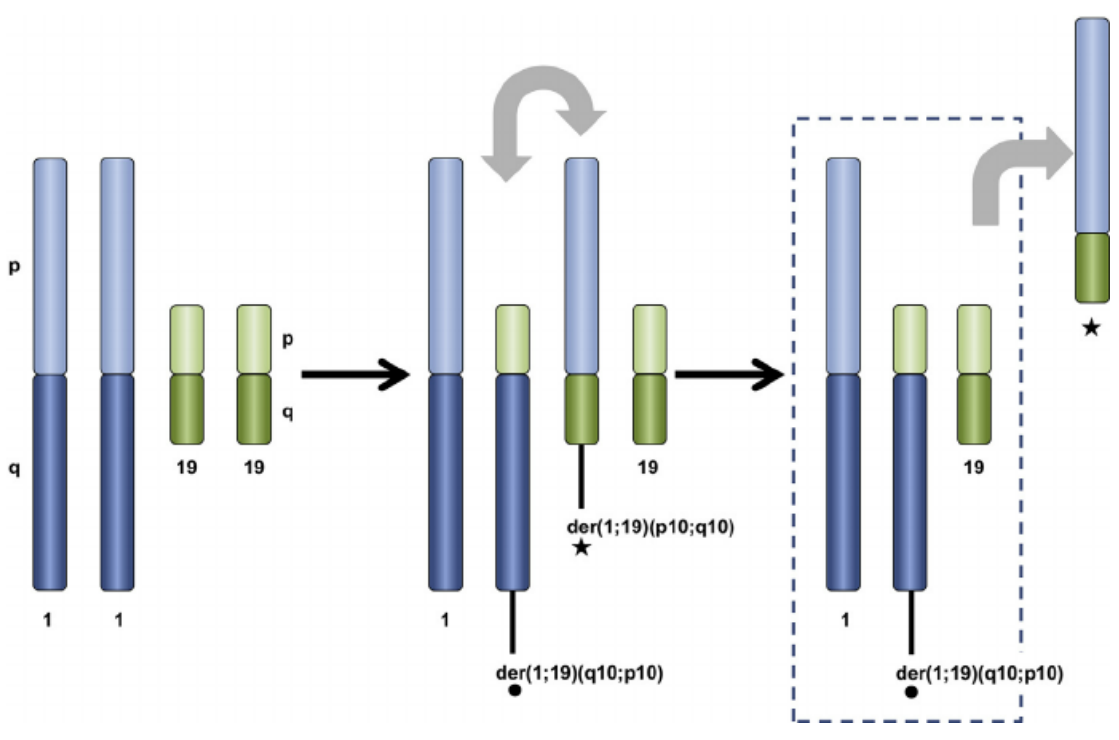

Figure 5 (A): Knudson's two-hit hypothesis applied to the CIC gene in 1p/19q co-deleted ODs. Yellow bars represent wild type CIC alleles on $19 \mathrm{q}$ arm. The first allele is lost (1st Hit) due to a reciprocal translocation between chromosomes 1 and 19. The second allele is disrupted (2nd Hit) by a somatic mutation (red-cross). With no functional CIC alleles remaining, the gene is effectively lost and tumorigenesis can begin. (B) $1 \mathrm{p} / 19 \mathrm{q}$ co-deletion accruing through whole-arm translocation between chromosomes 1 and 19 [t(1;19)(q10;p10)]. Two derivative chromosomes, $\operatorname{der}(1 ; 19)(\mathrm{p} 10 ; \mathrm{q} 10)[\star]$ and $\operatorname{der}(1 ; 19)(\mathrm{q} 10 ; \mathrm{p} 10)[\bullet]$ are generated, followed by loss of the derivative [ $\star$ ]. Figure adopted from Pinkham et al., 2015 [124], and Luke Webster (https://scienceplunge.wordpress.com/2015/03/31/investigatingcic-a-gene-associated-with-the-development-of-brain-cancer/). 


\subsubsection{Putative genes in $1 p 36$ and 19q13.3 region}

Mapping studies of chromosomal arms 1p36 and 19q13.3 in oligodendroglial tumours have revealed the presence of several putative and functionally related genes including tumour suppressor genes. Tumour suppressor genes at these regions either are deleted along with combined loss of $1 \mathrm{p} / 19 \mathrm{q}$, mutated or otherwise transcriptionally repressed or hypermethylated.

The CIC gene located on chromosome $19 \mathrm{q}$ and FUBPI gene located on $1 \mathrm{p}$ are well demonstrated examples. Several putative ODs suppressor genes have been reported to be located on $1 \mathrm{p}$, and it is proposed that at least one of these genes may contribute to the initiation of tumorigenesis in oligodendroglia therefore $\mathrm{LOH}$ on $1 \mathrm{p}$ and $19 \mathrm{q}$ is a favourable prognostic marker, [125]. The CIC gene located on chromosome 19q and FUBP1 gene located on 1p respectively: mutations in the CIC and FUBP genes [76]. EMP3 gene on 19q13.3 associated with retaining chromosome arms $1 \mathrm{p}$ and $19 \mathrm{q}$ in ODs - is aberrantly expressed or methylated [104] [105]. The functional loss of both alleles of EMP3 gene accounts for the reduced disease progression-free interval in OD-II patients [106]. NHE-1 on $1 \mathrm{p}$ is silenced in oligodendrogliomas secondary to $I D H$-associated hypermethylation and 1p allelic loss [107].

Detected 150-kb minimal deleted region on 1p36 and identified CAMTA1 as tumour suppressor gene at this region that was deleted in all oligodendroglial tumours with 1p deletion [112]. Moreover, the expression level of CAMTAl was low in ODs and was concordant with 1p deletion compared with those without $1 \mathrm{p}$ deletion. There exist several other potential geneses in this region reported in recent studies $[51,52]$.

\subsubsection{Clinical implications of $1 p$ and 19q co-deletion in oligodendrogliomas}

The combined loss of $1 \mathrm{p}$ and $19 \mathrm{q}$ is molecular hallmark of ODs diagnosis and prognosis. Since long $1 \mathrm{p} / 19 \mathrm{q}$ deletion has been considered a reliable marker and a standard of care molecular signature [126-128]. Despite the occurrence of $1 \mathrm{p} / 19 \mathrm{q}$ loss in tumours with atypical oligodendroglial or even pure astrocytic features; the typical oligodendroglial histological features are strongly associated with $1 \mathrm{p} / 19 \mathrm{q}$ loss, which is critically important as diagnostic point of view [129]. Given the presence of similar typical mutations in astrocytoma and GBM, the diagnosis of tumour subsets is well-supported based on their unusual profiles. Moreover, $1 \mathrm{p}$ and 
19q deletion serves as predictor of survival in ODs, astrocytomas and mixed oligoastrocytomas [129].

Comparable to combined $1 \mathrm{p} / 19 \mathrm{q}$ deletion, the $1 ; 19$ translocation is also associated with higher overall survival as well as progression-free survival in low-grade glioma patients [117]. Most importantly, 1p;19q translocation has so far not been observed in any other tumour type, and in contrast to other translocations the centromeric fusion of $1 \mathrm{q}$ and $19 \mathrm{p}$ does not result in an activated oncogene. This provides additional benefit for ODs diagnosis. The $1 \mathrm{p}$ and 19q deletion is not only a hallmark of diagnostic relevance but also has prognostic and predictive significance $[129,130]$. In patients with pure ODs the combined loss of $1 p$ and $19 q$ is a statistically significant predictor of prolonged survival, independent of tumour grade $[50,129,131]$. Genetic characteristics and molecular expression based classification of low grade gliomas correlate better with survival than achieved through histological classification [14, 15].

The combined $1 \mathrm{p} / 19 \mathrm{q}$ loss in ODs is clinically associated with a better response to chemotherapy as well as a favourable response to radiation therapy and increased survival of patients, particularly in relation to disease free interval [14, 132-135]. In fact, the evolution of molecular signatures of response to therapy has opened the ways for ODs treatment [132, 136]. Louis and colleagues provided a major breakthrough in the field with an observation that the loss of $1 \mathrm{p} / 19 \mathrm{q}$ in anaplastic ODs was correlated with $100 \%$ response rate to PCV chemotherapy, whereas in the absence of 1p/19q loss the response rate was 20-25\% [50]. Thus, the molecular analysis may aid therapeutic decisions and predict outcome in patients with ODs [50, 132, 135].

The prognostic value of $1 \mathrm{p} / 19 \mathrm{q}$ co-deletion in patients with overall survival was verified. The strong positive predictive value of this biomarker for overall was also shown for patients with co-deletion treated with neurosurgery and (RT) plus PCV vs. neurosurgery and RT alone [130]. Low-grade glioma represent an improved outcome after adjuvant PCV chemotherapy, thus making PCV chemotherapy now standard of care for all 1p/19q co-deleted tumours regardless of grade [83], and in many studies into the nature of the sensitivity of chemotherapy of $1 \mathrm{p} / 19 \mathrm{q}$ codeleted ODs [137]. In addition these studies allowed the molecular analysis of large prospective phase III trials conducted by RTOG and EORTC that explored the role of (neo)adjuvant PCV chemotherapy in anaplastic oligodendroglial tumours. These trials, although initially failed to 
show survival benefit from the addition of PCV to RT they already showed the major prognostic significance of combined 1p/19q loss [134, 138].

Benefit to PCV chemotherapy is not only limited to the $1 \mathrm{p} / 19 \mathrm{q}$ co-deleted; but potential other predictive factors such as 1p/19q co-deletion with IDH mutations and MGMT promoter methylation could be of additional value [83]. MGMT promoter methylation rates (80\%) in grade II and grade III glioma could be a marker of therapeutic response to TMZ in low-grade gliomas [139]. This unexpected prognostic finding after RT alone was considerably resolved with the discovery of IDH mutations, which were found in $60-80 \%$ of all grade II and III glioma, and in up to $100 \%$ in $1 \mathrm{p} / 19 \mathrm{q}$ co-deleted tumours in some studies. Not only were $I D H$ mutations found to be prognostic, they also strongly correlated to the presence of MGMT promoter methylation. First of all, this revealed that the significance of $M G M T$ promoter methylation in GBM differed from that in grade III glioma. In vivo models have suggested that the metabolic alterations present in $I D H$-mutated cells may induce a hypermethylated state, thus leading to an explanation of some of the findings in the clinical studies. At present, this G-CIMP appears to be a very strong prognostic factor for outcome in grade III tumours, and perhaps predictive for benefit to adjuvant chemotherapy.

Altogether, these observations suggest that genetic analysis may aid therapeutic decisions and predict outcome in patients with anaplastic ODs. Given the lack of $1 \mathrm{p} / 19 \mathrm{q}$ association in patients with astrocytic neoplasms and the previously demonstrated chemosensitivity of ODs, a combined approach of histologic and genotypic assessment could potentially improve existing strategies for patient stratification and management [129]. However there is little known with regard to how such genetic alterations correlate with more global patterns of gene expression in ODs. Albeit, ODs display histological malignant transformation accompanying events such as distinctive genetic and epigenetic profile with marked mutational heterogeneity; surprisingly it is not observed at tumour recurrence. This indicates that considerable spatiotemporal genetic heterogeneity in ODs does not result in rapid tumour progression [140]. However, in the light of genetic and epigenetic profiles with provided evidence (as discussed in above sections), demonstrate that genetic entities offer more restrictive diagnosis of oligodendroglial features and may help appropriate decision to therapy. 


\section{$1.4 \quad$ Non coding RNAs (ncRNAs)}

Non-coding RNAs (ncRNAs, also referred as non-protein coding RNAs) are comprised of 19-25 nucleotides (nt) and represent part of the genome that do not translate into proteins. The human genome contains only $\sim 20,000$ protein encoding genes, which is less than $2 \%$ of the whole genome. For almost two decades ago, most mapping projects have focused on the coding sequences, mainly because the noncoding part of the genome have long been considered as a "trash" ("junk DNA"). However, later the genomic regions comprising long repeated nucleotide sequences and their molecular interactions with transcription factors, prediction of binding sites as well as their role chromatin organization and gene expression gained focus of great interest due to their regulatory roles in living cells [141]. The ENCODE (Encyclopedia of DNA Elements) has revealed several aspects of non-coding part of the genome and statistics explain that more than $90 \%$ of the human genome contains functional ncRNA [142-147].

Based on their size, origin or mode of synthesis the ncRNAs are categorized into short ncRNAs and long ncRNAs (lncRNAs) [141]. Short ncRNAs include miRNA, small interference RNA (siRNA), small nuclear RNA (snRNA), small nucleolar RNA (snoRNA), transfer RNA (tRNA), Piwi-interacting RNA (piRNA), Y-RNA, vault RNA, and so forth [148-152]. In contrast the lncRNAs include long intergenic ncRNA (lincRNA) as well as $18 \mathrm{~S}$ and $28 \mathrm{~S}$ subunits of ribosomal RNA (rRNA) and among few others [148-150, 153, 154].

Ultraconserved genes (UCGs) also known as ultraconserved sequences (UCRs) are a new class of ncRNAs and serve as functionally active regulatory entities [155, 156]. UCRs by definition are those parts of human genome located within both intra- and intergenic regions that are absolutely conserved with $100 \%$ identity between orthologous regions of human, rat and mouse genomes [155]. These regions represent minimal loss of heterozygosity with no insertions or deletions, minimal regions of amplification, or common breakpoint regions in cancers [157]. Callin et al., identified certain UCRs whose expression is regulated by miRNAs that are abnormally expressed during human cancers [156]. In fact, the UCRs have distinct signatures and have been proposed direct targets of miRNAs (i.e. directly interact with miRNAs) which are altered in human cancers [156]. 


\subsubsection{MicroRNAs}

The miRNAs are the most putative class of ncRNAs and are attributed in regulation of gene expression. MiRNAs are 22 nucleotide single-stranded short ncRNAs which are expressed endogenously and regulate almost every cellular process through negative control on gene expression at the posttranscriptional level [158-162]. Functionally, miRNAs reduce the levels of many of their target transcripts, as well as the amount of protein encoded by these transcripts, by direct and imperfect interaction with messenger RNA (miRNA:mRNA) [160, 163, 164]. miRNA-mediated mechanism for the posttranscriptional restriction of extensive evolutionarily conserved genes such as those involve in vertebrate development can repress the expression of their natural targets through mRNA cleavage in addition to inhibition of productive translation [165].

Initially miRNAs were identified in Caenorhabditis elegans (C. elegans) [166-168], and their role was demonstrated in regulation of developmental processes [169-173]. Approximately 30\% of all protein coding genes are regulated by miRNAs [162, 174]. It has been shown that most of the miRNA genes are located at fragile sites as well as in minimal regions of LOH, minimal regions of amplification, or common breakpoint regions [157]. They are precisely regulated during neurodevelopment, participating in several cellular pathways including proliferation, apoptosis and cell migration and behave as tumour suppressor genes or oncogenes. MiRNAs are expressed in a tissue-specific manner and are considered to play important roles in cell proliferation, apoptosis and differentiation during mammalian development [158, 169, 175]. Given that these ncRNAs are expressed in a highly tissue-specific manner and in specific cellular compartments, and due to their heterogeneous mechanisms of action, it is a challenge to determine the diverse regulatory functions of these transcripts in many disease models, and this is a truly exciting field of research.

The functional mature miRNA is generated after various stages of post-transcriptional processing of pri-miRNA [176]. MiRNAs are encoded by genes that are transcribed into precursor miRNAs (primiRNAs), which are sequentially cleaved transcripts in the nucleus about 80nt, called premiRNAs. MiRNAs are transcribed as long primary transcripts (pri-miRNAs) and are processed in the nucleus by the enzyme Drosha [177]. miRNAs typically are excised from a 60-nt to 110-nt 
hairpin precursor (fold-back) RNA structure (pre-miRNA) that is transcribed from a larger primary transcript (pri-miRNA) [178]. In turn, the pre-miRNAs are transported to the cytoplasm by Exportin-5 (Exp5) [179, 180], where they are processed into mature miRNAs by enzyme Dicer [176]. MiRNAs are then incorporated into a ribonucleoprotein complex called RNAinduced silencing complex (RISC) (Figure 6). MiRNAs act through the RISC complex which directs/traffics them to the target mRNA in order to govern the regulation of gene expression, either through cleavage of mRNA, or by repression of its translation.

Once processed into their mature form, they bind to complementary sequences in the 3 ' UTR (un-translated region) of specific genes. Alternatively, they may also bind to other regions of the gene, including 5' UTR region as well as to coding regions. Thus, by binding to 3' UTR region of the target mRNA, they modulate the expression of a variety of mRNAs and proteins either by mRNA degradation or repression of mRNA translation. 


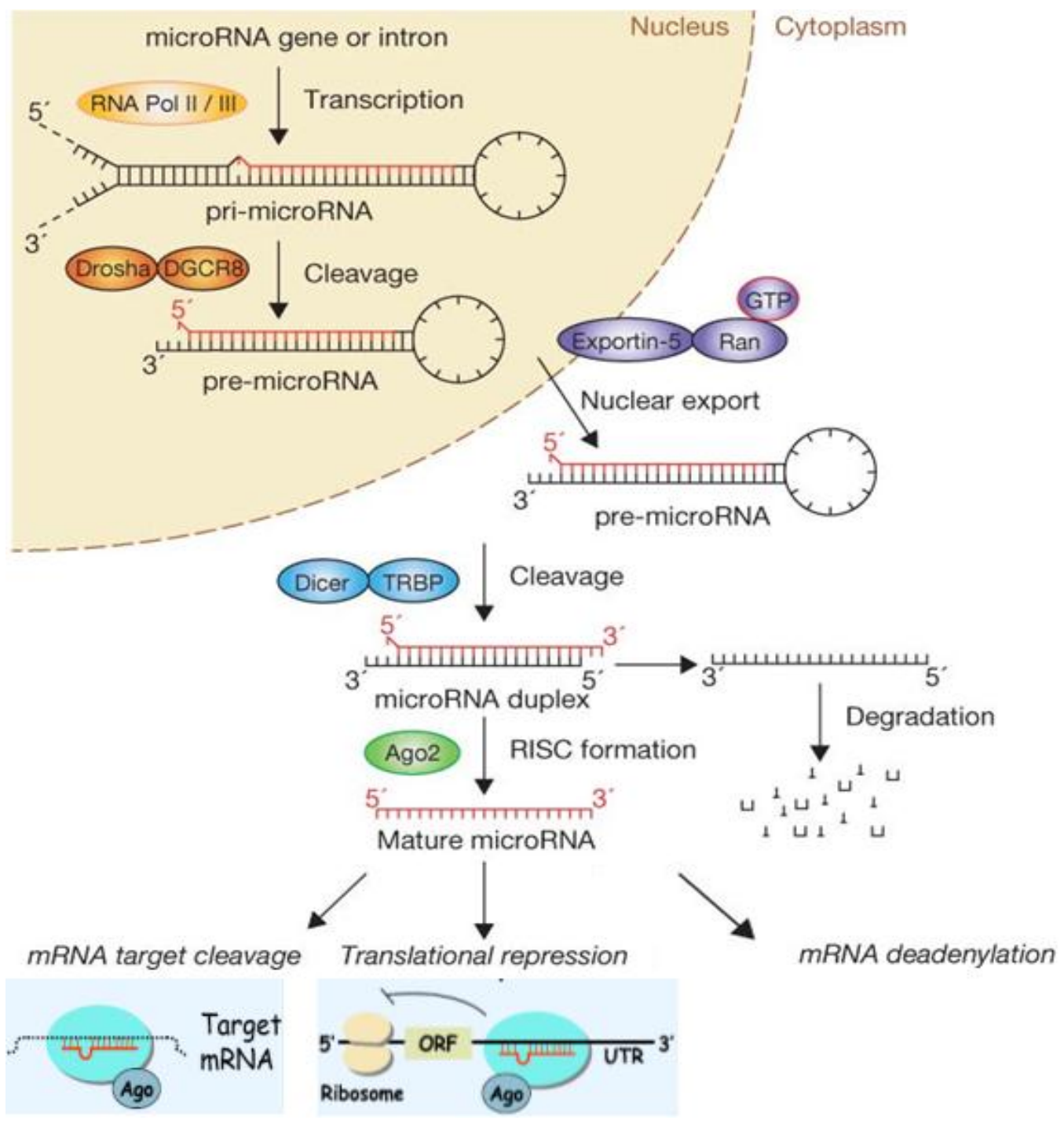

Figure 6: miRNA biogenesis and processing: miRNA genes are transcribed as precursor miRNAs (pri-miRNAs) in the nucleus and are processed/ cleaved into 80nt transcript called premiRNAs by Drosha. The pre-miRNAs are transported to the cytoplasm by Exportin-5 (Exp5) and processed into mature miRNAs by enzyme Dicer in the cytoplasm. Mature miRNAs are then engaged in ribonucleoprotein complex called RNA-induced silencing complex (RISC) which guide them to the target mRNA in order to govern the regulation of gene expression, either through cleavage of mRNA, or by repression of its translation. (Adopted and modified from [181] and pang et al., [182]). 


\subsubsection{Roles in cancer initiation progression and metastasis}

For the past two and a half decades, the causes of tumorigenesis have been considered as a result of genetic and/or epigenetic alterations in protein-coding oncogenes and/or tumour suppressor genes $[183,184]$. However, the discovery of genes which produce short ncRNA transcripts i.e. miRNAs with no functional open reading frame, has clarified alternative ways of gene regulation. In 1993, Ambros and colleagues discovered a gene (Lin 4) who's gene product was a small non-protein coding RNA that negatively regulates the level of LIN-14 protein and affect the developmental process in C. elegans [166].

Calin and colleagues for the first time pioneered the idea that miRNAs are involved in human tumorigenesis initially reported in chronic lymphocytic leukaemia [185-187], and clarified that the genomic complexity of the cancer is far greater than expected before $[156,187]$. Based on these pioneering studies, several other investigations revealed that miRNAs are involvement in regulatory processes during development of various cancer types [182, 184, 188-195]. Assuming that a large fraction of ultra-conserved non-coding regions (UCRs) encode a particular set of ncRNAs whose expression is altered in human cancers; the UCRs may serve as distinct signature in human cancers [156]. This study identified certain UCRs whose expression is regulated by miRNAs that are abnormally expressed during human cancers. In addition it was proposed that the inhibition of an overexpressed UCR induces apoptosis in colon cancer cells [156].

During the passage of tumorigenesis the expression level of miRNAs either is increased significantly or decreased from corresponding normal tissue, while some remain unchanged [196-199]. This implies that miRNA expression is correlated with bio-pathologic features of cancer progression as well as the functional relevance in tumorigenesis [197, 199-201]. The deregulation of miRNAs involved in processes such as cell proliferation, apoptosis, regulation, invasion, cell cycle arrest, invasive cell behaviour and angiogenesis. These findings support the notion that copy number alterations of miRNAs and their regulatory genes are highly prevalent in cancer and may account partly for the frequent miRNA gene deregulation reported in several

tumour types [202]. The overexpression of miRNAs may function as oncogenes and promote cancer development by negatively regulating tumour suppressor genes, whereas down regulation of some miRNAs in various human cancers, indicate that they act as tumour suppressors [185, 
203]. The miRNAs while acting as tumour suppressors might repress the expression of important cancer-related genes [182, 184, 192, 203], and may act as either dominant or recessive cancer genes [204]. This is evident that miRNAs can target oncogenes and subsequently modulate cancer pathways, thus acting as tumour suppressors [164, 184, 192, 194, 195, 203].

In addition to short ncRNAs the lncRNA have also been attributed in cancer development [152, 205-211]. The lncRNAs such as HOX antisense intergenic RNA (HOTAIR) and MALAT1 have been shown to be associated with cancer development [208]. HOTAIR is expressed on chromosome 12 and controls the expression of genes on chromosome 2 and is attributed in cancer metastasis [206, 212]. Interestingly bifunctional lncRNAs have been described that may perform dual function-that is they may mimic the functions of mRNA and ncRNAs, or otherwise they may reflect two separate functions of ncRNAs [151, 213]. The lncRNAs can affect imprinting or gene expression in cis and have been shown to silence from three to ten genes in cis in imprinted gene clusters [214], that may have role in diseases including cancer. Another class of lncRNA-the telomeric repeat containing RNA (TERRA) transcript is associated with telomere shortening and compromised telomere function which contribute to telomere syndromes and cancer [215].

Differential expression in cancer subsets may help understanding progression [190]. MiRNAexpression profiling of human tumours has identified signatures associated with diagnosis, staging, progression, prognosis and response to treatment in human malignancies. In addition, profiling has been exploited to identify miRNA genes that might represent downstream targets of activated oncogenic pathways, or that target protein-coding genes involved in cancer [216]. Poorly differentiated human tumours could be successfully classified using miRNA expression profiles [217]. Differential expression gives the notion that miRNAs involvement cancer as miRNA expression could differentiate micro- from macro-adenomas and treated from nontreated patient samples, improving the classification of tumours [218]. The implications of these findings could be experienced for the diagnosis and treatment of cancer. 


\section{5 microRNAs in oligodendrogliomas}

Expression profiling has unveiled the miRNA signatures that not only distinguish brain tumours from normal tissues, but can also differentiate histotypes or molecular subtypes with altered genetic pathways $[7,13]$. Tumours of central nervous system have complex expression patterns. Some of the miRNAs are tissue specific and few are housekeeping and exhibit series of spatial temporal expression patterns. For instance some miRNAs are brain specific miRNAs [219-223]. Spatial temporal expression analysis of miRNAs demonstrates that some miRNAs are more strongly expressed in neurons than in astrocytes, while others are more specific to dendrites [222-225].

Recent data suggests that miRNAs like other tumours also serve as oncogenes in brain tumours and are extensively deregulated in malignant gliomas [182, 226, 227]. In brain tumours, it has been shown that miRNAs not only repress the translation of target mRNA but they could also stimulate translation of some mRNAs, thus acting as both positive as well negative regulators of glioma progression [228]. During the course of glioma progression the deregulated miRNAs participate in different pathways and processes [228-230]. There has been proposed several miRNAs in glioma progression, however, miRNA-21 is one of the most frequently identified in high grade malignant gliomas; regulating key pathways of malignancy either acting as oncomiR or tumour suppressor and holds a diagnostic and prognostic value as well as serves as a potential therapeutic target [200, 231-239].

The regulatory roles of miRNAs in gliomas just have begun less than a decade ago and their role is demonstrated through participation in tumour related major pathways, cell cycle regulation, proliferation and angiogenic pathways [230, 240-246]. Functional relevance of miRNAs in glioma progression could be identified by integrative genome analysis of miRNA/mRNA profiles [247]. The better understanding of miRNA biology suggests that miRNAs could be utilized as therapeutic targets [248-251]. Several potential miRNAs have been suggested as diagnostic and prognostic markers [235, 237, 249, 252-255]. As compared to ODs, the low-grade astrocytomas exhibit a higher expression of genes related to mitosis, replication, and inflammation [22]. Additionally, the low-grade astrocytic tumours represent miRNA profiles similar to those previously described for GBMs [7, 22]. 
Until recently the information regarding the expression of miRNAs in oligodendroglial tumours has not been well appreciated and is limited. Nelson and colleagues performed first profiling study in ODs using RNA-primed, array-based, Klenow Enzyme (RAKE) miRNA microarray platform in conjunction with Locked Nucleic Acid (LNA)-based in situ hybridization (LNAISH) for ODs and archival FFPE human brains [256]. Comparative miRNA expression analysis was performed in 20 ODs and 21 samples of non-neoplastic brain tissue (9 foetuses). Hierarchical clustering of deregulated miRNAs was able to separate all tumours from nontumour tissues.

Significantly elevated expression of miR-9 was observed in ODs and brains of foetuses compared to the cerebral tissue of adult [256]. This suggests that miR-9 upregulation could play important role in ODs development. This could be due to miR-9 being normally expressed mainly in glial cells, and the increase in gliomas being due only to an increase of the density of cells of glial lineage. However, in situ hybridization showed low levels of miR-9 expression in adult neurons, but higher levels to proliferating neuroblasts as well as glioblasts in foetal brain and also in tumour cells [256]. Interestingly, miR-9 is a brain specific miRNA that is $100 \%$ conserved between known mammalian, zebrafish, chicken, drosophila, and anopheles species, with a somewhat lower degree of conservation with C. elegans [256]. Additionally, the same study demonstrated that miR-124 expression level is reduced in ODs as compared to normal brain [256]. In rodent OD-initiating cells, the down regulation of both miR-124 and miR-137 has been observed. One interesting aspect, the loci of 3 Argonauts genes are mapped to 1p, whose region is deleted up to about $60 \%$ of ODs. In order to assess whether the loss of the Argonauts genes could affect the expression of miRNAs, four ODs were evaluated, two of which harboured chromosome 1 deletion, but no significant difference in miRNA expression levels were observed among the tested samples. It could be speculated that decreased levels of miR-124a might correlate with neoplastic transformation.

However, in situ hybridization provides direct evidence against this hypothesis because miR$124 \mathrm{a}$ is only expressed in neurons but not in tumour cells. Therefore, the low levels of miR-124a expression by microarrays in tissues derived from ODs might be due to these residual neurons [256]. MiR-124 and miR-137 have been attributed in differentiation of glioma initiation stem cells. It was shown that miR-124 and RNA-137 efficiently induce differentiation of adult mouse 
neural stem cells, mouse $\quad$ ODs derived stem cells and human GBMderived stem cells and induce GBM cell cycle arrest [257]. Recently, it was shown that miR-137 is differentially expressed in oligodendroglial tumours likewise; downregulated in grades II ODs and III anaplastic ODs [258]. This indicates that miR-137 expression is associated with tumour grade. Low expression of miR-137 was associated with shorter progression-free survival and overall survival. Functional validation revealed that miR-137 inhibits tumour growth, cell viability and invasion of ODs in vitro by targeting CSE1L [258].

Lages and colleagues performed differential expression of the 282 miRNAs in order to investigate differential expressional levels between ODs and GBM compared to control brain tissues. 26 miRNAs were deregulated as compared to the expression patterns of control tissues. Expression levels of seven miRNAs (miR-21, miR-128, miR-132, miR-134, miR-155, miR-210 and miR-409-5p) and their target analysis showed discrimination of grade II and III ODs from grade IV GBM [259]. Lau et al., have reported a consistent reduction of miR-9 expression during oligodendrocytes differentiation [260]. These observations indicate that miR-9 expression is important in the developmental regulation of ODs, however the mechanisms for such regulation are not known. Additional reports have clarified that ODs exhibit a significantly reduced level of miR-210 as compared with normal brain tissue. In contrast, astrocytic tumours demonstrate significantly increased levels of miR-210. Moreover, the expression of miR-210 is positively correlated with the grade of astrocytic tumour, in the following order: grade IV > grade III > grade II > normal brain tissue [261]. Such observations suggest that low-grade tumours can be clearly delineated from GBM by miRNA measurements as well as mRNAs expression analyses [262].

Alterations in miRNA expressions are found in early stage of cancer, whereas differential expression could detect cancer grades and malignancy. Therefore miRNA profiling may serve as diagnostic and prognostic tool for glioma patients [251]. Expression profiling has unveiled miRNA signatures that not only distinguish brain tumours from normal tissues, but can also differentiate histotypes or molecular subtypes with altered genetic pathways [182]. Therefore, a small number of miRNA markers might be sufficient to identify glioma subtypes as well as discrimination among grades [7, 13, 22, 262]. 
AIMS AND OBJECTIVES 


\section{AIMS AND OBJECTIVES}

Sharp stratification and concordant classification of low grade diffuse gliomas based on histopathological analysis remains an impeding hurdle. Since miRNA expression profiling serves as signatures for identifying tumours and serves as signatures for identifying tumours $[184,187]$; poorly differentiated low grade tumours could be successfully classified and delineated using molecular profiles [217]. Differential expression could differentiate different grades, and may improve the classification of tumours [218]. There is limited evidence of miRNA expression profiling in different grades of ODs and differential expression between grade II and grade III ODs has not been performed.

The current thesis aimed to perform differential expression profiling of miRNAs and genes in grade II and grade III ODs and their correlation with 1p/19 q deletions. The more specific objectives are;

1. Performing microarrays to identify differentially expressed miRNAs and genes in grade III versus grade II oligodendrogliomas

2. Performing miRNA targets analysis in grade II and grade III

3. Correlation between downregulated miRNAs and their upregulated mRNA targets and vice e versa

4. Validation of miRNAs and mRNAs by real time qPCR

5. Validation of some putative miRNAs in cell lines 
METHODOLOGY 


\section{MATERIAL METHODS}

\subsection{Patient samples}

Frozen tumour tissue fragments were used from patients operated for ODs. 14 cases of ODs i.e. OD-II and anaplastic OD-II (7 cases per grade) and 15 non neoplastic white matter (nnWM) samples after microdissection with no previous history of treatment between 2002 and 2007 were included in the study. Non neoplastic white matter of temporal lobe epilepsy operated patients was used as control tissues. Samples were obtained from Tumour Bank, in accordance with the rules the Research Ethics Committee of the Institution (Proc. No. 8730/08). All samples were microdissected as previously described [263]. In each grade of ODs (II and grade III ODs), 8 samples were used; comprising 7 patients in each category and another one to serve as internal control. Samples were used in duplicate of each case of grade II and grade III ODs.

Mean age of patients was 45 years \pm 10.86 (33 to 57 years) and 39 years \pm 4.93 (33 to 46 years) of patients with ODs grades II and III, respectively. The mean age of the controls (patients with refractory epilepsy) was 36.7 years \pm 6.4 (median 37 years), 9 female subjects.

\subsection{Extraction and quantification of total RNA}

The extraction of total RNA was performed by Trizol (Invitrogen) as previously described [264, 265]. After the extraction, the total RNA was assessed by electrophoresis on agarose gel and quantification was achieved in $1 \mu \mathrm{l}$ of the sample, using NanoDrop 1000 spectrophotometer (ThermoScientific $\AA$ ). The remaining RNA was stored at $-70^{\circ} \mathrm{C}$ freezer until the use for microarray analysis.

\subsection{Microarrays for mRNA evaluation}

The samples (or pools) of RNAs used in mRNA microarray assays were additionally treated with DNAase and subjected to column purification (RNeasy, Qiagen $\left.{ }^{\circledR}\right)$. Analysis of gene expression profile was performed with the Agilent oligonucleotides microarray platform through the kit "Whole Human Genome Oligo Microarray" (Agilent ${ }^{\circledR}, \mathrm{G} 4112 \mathrm{~F}$ ) containing sequences of over 41,000 transcripts of genes. The complementary RNA (cRNA) labeled with the fluorochrome was generated from 200 ng RNA, using the kit "Quick Amp Labelling, one-color" (Agilent®, 5190-0442), as described previously [266]. 


\subsection{Microarrays for microRNAs profiling}

The samples were subjected to an additional extraction step with chloroform before being used in miRNA microarray, using the Agilent microarray platform with the kit oligonucleotide "Human miRNA Microarray" (Agilent®G4470B) containing sequences of 723 miRNAs. Briefly, after obtaining and quantification of the total RNA, $100 \mathrm{ng}$ of each sample were used to label RNA with the fluorochrome, using the kit "miRNA Labelling Reagent and Hybridization" (Agilent®, 5190-0408), according the manufacturer's instructions, as described previously [266].

\subsection{Analysis of microarray data}

Briefly, the slides were scanned $(535 \mathrm{~mm}$, resolution of $5 \mathrm{um} /$ pixel) with the help of the GenePix 4000B scanner and the program "GenePix Pro 6.0" (Molecular Devices®, Sunnyvale, CA, USA). The images were analysed using the program "Agilent Feature Extraction" and different transcriptomes (both mRNAs and miRNAs) were normalized to the expression value equivalent to the $75 \%$ percentile. The sets of transcripts (or miRNAs) differentially expressed between samples of grade II and grade III ODs were determined based on unpaired two-tailed Ttest using "R", considering $\mathrm{P}<0.05$.

\subsection{Differential expression analysis}

The $l m \_$fun (linear model) function was created in $\mathrm{R}$ environment to adjust linear models for each gene available in miRNApp and mRNApp objects. The terms of each gene are adjusted as a function of available groups (OD3, OD2 and nnWM). To adjust the comparisons of interest empirical Bayes method was employed and inference method was applied to increase the robustness of the differential expression. Given the fact there was a natural ordering of groups (nnWM, OD2 and OD3), the following comparisons were made: OD2 vs nnWM; and OD3 vs nnWM. OD3 was compared with OD2 and miRs and mRNAs from ODs (OD3 and OD2) were compared with white matter (nnWM).

Selecting as candidates to be differentially expressed genes should have adjusted p-values less than $5 \%$, and differences in the expression of at least $50 \%$ (which, in the log-ratio scale is represented by $\log _{2}(1.5)$ ). These data were visualized by generating venn diagrams and heatmaps using " $R$ ". 


\subsection{Determination of relationship between potential microRNAs and mRNA-targets}

The tables containing the miRNAs and the predicted targets were obtained by bioinformatics tools (http://www.microrna.org/microrna/getDownloads.do) in order to relate the miRNAs and mRNAs differentially expressed between grade II ODs and III. Furthermore, we used many programs with already validated algorithms that allow the prediction of potential targets of miRNAs in humans, such as DIANA, miRanda, TargetScanS, miRtarget and PicTar.

\subsection{Validation of miRNA and gene expression by Real-Time PCR}

The selection of differentially expressed miRNAs (4 highest and 6 lowest expressed) is made by correlating complementary mRNAs and according to the score $(\leq 0.1$ indicates high probability of miRNA / mRNA ratio) obtained by mirSVR tool [267]. The validation of expression analysis obtained by arrays was performed as previously described [266]. Reverse transcription was performed using TaqMan MicroRNA Reverse Transcription ${ }^{\circledR}$ kit according to the manufacturer's specifications. In RT-qPCR validation equipment the ABI PRISM 7500 Sequence Detection System (Applied Biosystems®) was used. The tests for the validation of miRNAs were performed with TaqMan MicroRNA Assays kit (Applied Biosystems®) and the RNU48 as endogenous control. All reactions were performed in duplicate by TaqMan system (Applied Biosystems $\left.{ }^{\circledR}\right)$.

The $\beta$-actin gene and GUS ( $\beta$-glucuronidase) were used as endogenous control to validate the

mRNAs. The results were calculated by the formula $2^{-\Delta \Delta \mathrm{Ct}}$. The GraphPad Prism 4.0 was used to calculate statistically significant differences. The Mann-Whitney test was used for comparisons between two groups and Kruskal-Wallis test for multiple groups, followed by Dunn's post-test.

\subsection{Establishing primary cell lines of oligodendrogliomas}

Since the commercial cell lines for OGs are not available and there was need to perform functional studies to validate the functional level, biological roles and miRNA/mRNA interactions, we performed trypsinization of some tumour samples during the time of sampling and plating them in rich culture media of foetal bovine serum, as described previously [268]. 


\subsection{Validation of miRNA and genes in cell lines}

The hsa-miR-23a and hsa-miR-27a functions were tested in BJ cells (human adult fibroblasts) by theClick iT® EdU Cell Proliferation Assay and FITC Annexin V apoptosis detection. 


\section{RESULTS}




\section{$4 \quad$ RESULTS}

\subsection{Differentially expressed microRNAs}

The microarray data analysed using " $R$ " program (an environment freely available from Bioconductor for analysing huge data sets. Data was visualized by generating Venn diagrams and heatmaps. The Venn diagrams below show the number of candidate miRNAs and genes differentially expressed between grade III and grade II oligodendroglia's when the comparisons was made in each of the indicated circles. We compared OD3 with OD2 as well ODs with nnWM (i.e. OD3-OD2; ODs-nnWM). Of the total 723 miRNAs evaluated by microarray, 214 miRNAs were upregulated and 217 were downregulated in all comparisons collectively. Specifically 43 miRNAs were downregulated and 50 miRNAs were overexpressed in OD3 (compared to OD2). Conversely, the 7 miRNAs showed reduced expressions in OD2 (compared to nnWM). 3 common miRNAs were down regulated in comparisons of both groups (OD3 vs OD2, and OD2 vs nnWM) (Figure 7).

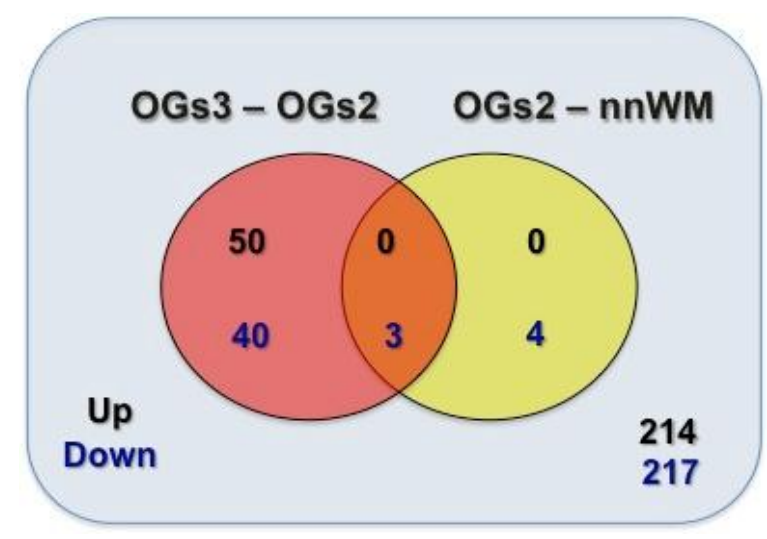

Figure 7: Number of miRNAs differentially expressed between grade III and grade II oligodendrogliomas and non-neoplastic white matter. OGs2: grade II oligodendrogliomas; OGs3: grade III oligodendrogliomas; nnWM: non neoplastic white matter. 


\subsection{Differentially expressed mRNAs}

Of the 41,000 mRNAs transcripts evaluated, 3285 genes were overexpressed and 3305 were downregulated in OD3 (when compared with OD2). Conversely, 19 genes were overexpressed and 117 were down regulated in OD2 (when compared to nnWM). 2 over expressed were common in OD2 and OD3, whereas among downregulated 6 were common in both grades (Figure 8).

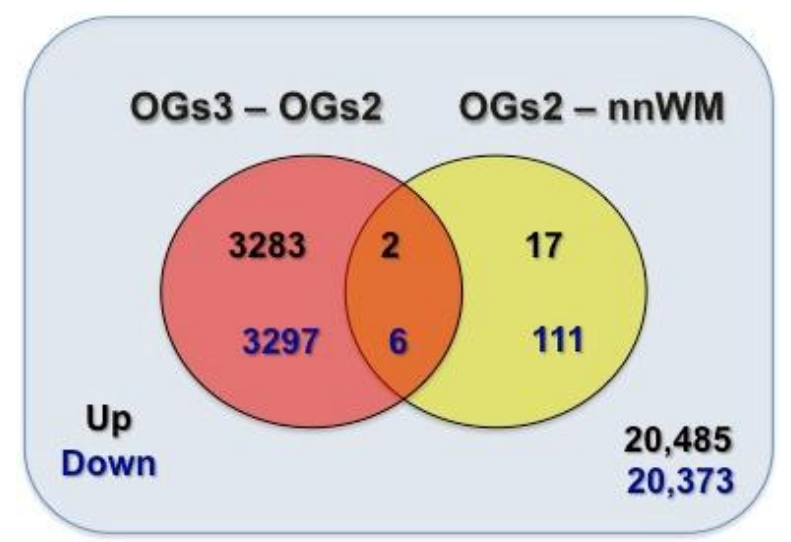

Figure 8: Number of mRNAs differentially expressed between grade III and grade II oligodendrogliomas, and non-neoplastic white matter. OGs2: grade II oligodendrogliomas; OGs3: grade III oligodendrogliomas; nnWM: non neoplastic white matter. 


\subsection{Top differentially expressed miRNAs and mRNAs in grade II and grade III ODs}

Of these several differentially expressed miRNAs and mRNAs (genes) from above comparison, we present here only the top expressed candidates. For convenience, function "showTopTable" was created using ' $\mathrm{R}$ ' to present the results obtained by above comparisons. This function represents the name of the miRNA/mRNA, log-ratio, average expression and adjusted p-value as shown in the tables below (Tables 3-6). The candidates for differentially expressed miRNAs/genes have adjusted $p$-values $<5 \%$, and differences in the expression of at least $50 \%$ (which, in the log-ratio scale is represented by $\log _{2}(1.5)$ ).

Table 3. Top differentially miRNAs expressed in grade II oligodendrogliomas

\begin{tabular}{cccc}
\hline miRNAs & $\operatorname{logFC}$ & AveExpr & adj.P.Val \\
\hline hsa-27 & -0.8282 & 10.691 & 0.01544 \\
\hline hsa-92b & -1.2136 & 8.220 & 0.01544 \\
\hline hsa-30a & -0.6529 & 7.992 & 0.04744 \\
\hline hsa-708 & -0.7997 & 7.156 & 0.04744 \\
\hline hsa-9 & -0.7416 & 14.072 & 0.04744 \\
\hline hsa-23a & -0.7043 & 11.129 & 0.04872 \\
\hline hsa-100 & -0.8572 & 11.690 & 0.04872 \\
\hline
\end{tabular}


Table 4. Top differentially miRNAs expressed in grade III anaplastic oligodendrogliomas

\begin{tabular}{|c|c|c|c|}
\hline miRNAs & $\log \mathrm{FC}$ & AveExpr & adj.P.Val \\
\hline hsa-497 & -2.3710 & 10.741 & $5.366 \mathrm{e}-11$ \\
\hline hsa-195 & -2.3487 & 11.541 & $6.901 \mathrm{e}-09$ \\
\hline hsa-9 & -2.0643 & 14.512 & $1.701 \mathrm{e}-08$ \\
\hline Has-130a & -2.4763 & 10.853 & $2.624 \mathrm{e}-08$ \\
\hline hsa-584 & 2.6173 & 8.040 & $7.838 \mathrm{e}-08$ \\
\hline hsa-151-5p & 1.7392 & 10.985 & $1.352 \mathrm{e}-07$ \\
\hline hsa-27b & 2.0654 & 11.662 & $1.414 \mathrm{e}-07$ \\
\hline hsa-23b & 2.0655 & 11.529 & $1.949 \mathrm{e}-07$ \\
\hline hsa-338-3p & 3.4978 & 12.716 & $9.268 \mathrm{e}-07$ \\
\hline hsa-330-3p & 1.9877 & 7.627 & $4.064 \mathrm{e}-06$ \\
\hline hsa-340 & 1.2047 & 9.872 & $1.441 \mathrm{e}-05$ \\
\hline hsa-let-7c & -0.9091 & 13.257 & $4.595 \mathrm{e}-05$ \\
\hline ebv-BART12 & -2.378 & 87.944 & $5.694 \mathrm{e}-05$ \\
\hline hsa-19b & -1.1162 & 12.244 & $7.471 \mathrm{e}-05$ \\
\hline hsa-135a & -1.7538 & 9.323 & $1.616 \mathrm{e}-04$ \\
\hline hsa-219-5p & 2.3953 & 14.266 & $1.914 \mathrm{e}-04$ \\
\hline hsa-219-2-3p & 2.0655 & 9.706 & $2.191 \mathrm{e}-04$ \\
\hline hsa-100 & 1.2412 & 11.690 & $3.193 e-04$ \\
\hline hsa-96 & -3.0952 & 8.250 & $3.711 \mathrm{e}-0$ \\
\hline
\end{tabular}


Table 5. Top differentially expressed genes in grade II oligodendrogliomas

\begin{tabular}{|c|c|c|c|c|}
\hline Accession No. & Genes & $\log \mathrm{FC}$ & AveExpr & adj.P.Val \\
\hline NM_003494 & DYSF & 1.2532 & 7.324 & 0.01478 \\
\hline NR_026908 & AQP4-AS1 & -2.0166 & 5.706 & 0.01478 \\
\hline NM_145053 & UBQLNL & -1.5993 & 5.864 & 0.01478 \\
\hline BC040210 & AKR1C1 & -1.4303 & 5.352 & 0.01478 \\
\hline BC005052 & MARVELD3 & -2.0703 & 7.934 & 0.01478 \\
\hline NM_020297 & ABCC9 & -1.1013 & 5.371 & 0.01478 \\
\hline AL136541 & GPR98 & -2.0770 & 7.010 & 0.01478 \\
\hline NM_178824 & WDR49 & -2.0905 & 5.981 & 0.01478 \\
\hline NM_000216 & KAL1 & -2.1533 & 8.061 & 0.01478 \\
\hline NM_004105 & EFEMP1 & -1.8172 & 11.154 & 0.01478 \\
\hline NM_006074 & TRIM22 & -1.5145 & 7.285 & 0.01500 \\
\hline NM_017702 & DEF8 & 0.8419 & 8.627 & 0.01804 \\
\hline NM_007274 & ACOT7 & 0.9806 & 8.823 & 0.01860 \\
\hline NM_180989 & GPR180 & 0.9276 & 6.430 & 0.01860 \\
\hline NM_003759 & SLC4A4 & -1.6364 & 8.253 & 0.01860 \\
\hline NM_003064 & SLPI & -1.5590 & 6.564 & 0.01860 \\
\hline AK130099 & LINC01094 & -2.7889 & 8.120 & 0.01860 \\
\hline
\end{tabular}


Table 6. Top differentially expressed genes in grade III anaplastic oligodendrogliomas

\begin{tabular}{|c|c|c|c|c|}
\hline Accession No. & Genes & $\log \mathrm{FC}$ & AveExpr & adj.P.Val \\
\hline NM_003107 & SOX4 & -4.130 & 11.568 & $5.436 \mathrm{e}-08$ \\
\hline NM_003494 & DYSF & 2.951 & 7.324 & $9.527 \mathrm{e}-08$ \\
\hline NM_177995 & PTPDC1 & 2.865 & 7.681 & $1.206 \mathrm{e}-07$ \\
\hline AK023623 & GLDN & 2.677 & 5.589 & $2.005 \mathrm{e}-07$ \\
\hline NM_144605 & SEPT12 & 3.053 & 6.276 & $2.005 e-07$ \\
\hline NM_173160 & FXYD4 & 2.445 & 5.277 & $2.052 \mathrm{e}-07$ \\
\hline NM_015123 & FRMD4B & 3.126 & 6.477 & $2.785 e-07$ \\
\hline NM_006636 & MTHFD2 & -3.931 & 10.401 & $2.785 \mathrm{e}-07$ \\
\hline ВC087859 & LOC401317 & 2.546 & 5.452 & $2.785 \mathrm{e}-07$ \\
\hline NM_000574 & CD55 (DAF) & 2.046 & 6.106 & $2.785 \mathrm{e}-07$ \\
\hline AK026467 & CD22 (BL-CAM) & 3.322 & 5.343 & $2.802 \mathrm{e}-07$ \\
\hline NM_025244 & TSGA10 & 3.192 & 5.988 & $2.802 \mathrm{e}-07$ \\
\hline NM_007249 & KLF12 (AP-2 alpha) & -2.703 & 9.726 & $2.999 \mathrm{e}-07$ \\
\hline NM_006257 & PRKCQ & 3.319 & 5.819 & $3.314 \mathrm{e}-07$ \\
\hline NM_020209 & SHD (SH2 or SHP-2) & -5.565 & 12.369 & $3.993 \mathrm{e}-07$ \\
\hline NM_001093725 & MEX3A & -3.548 & 9.583 & $4.309 \mathrm{e}-07$ \\
\hline NM_170693 & SGK2 & 3.255 & 5.372 & $5.200 \mathrm{e}-07$ \\
\hline NM_014603 & CDR2L (CDR2) & 2.142 & 8.994 & $5.307 \mathrm{e}-07$ \\
\hline
\end{tabular}




\subsection{Assessment of differentially expressed miRNAs and mRNAs}

Heatmaps were generated for only those miRNAs and mRNAs that were identified as candidates for differential expression between three groups (ODs2, ODs3 and nnWM). Almost all patient samples in OD3 (brown) are showing clearly distinct miRNA and mRNA expression patterns different from nnWM (purple) samples. Similarly OD2 (green) samples showed different patterns than nnWM (Figure 9 and 10). Surprisingly, the sample 678, initially identified as OD2, has a miRNA signature compatible with the category of OD3 individuals. Initially it gave us impression that this might be described as a border line between OD2 and OD3 or might have some unique feature that need to be further investigated. However, this was also suspected that there might be an error during classification of samples. Later on, careful re-evaluation by pathological features revealed that this sample belongs to astrocytoma but not OD2. However, miRNA profile of this patient sample was closer to OD3. 


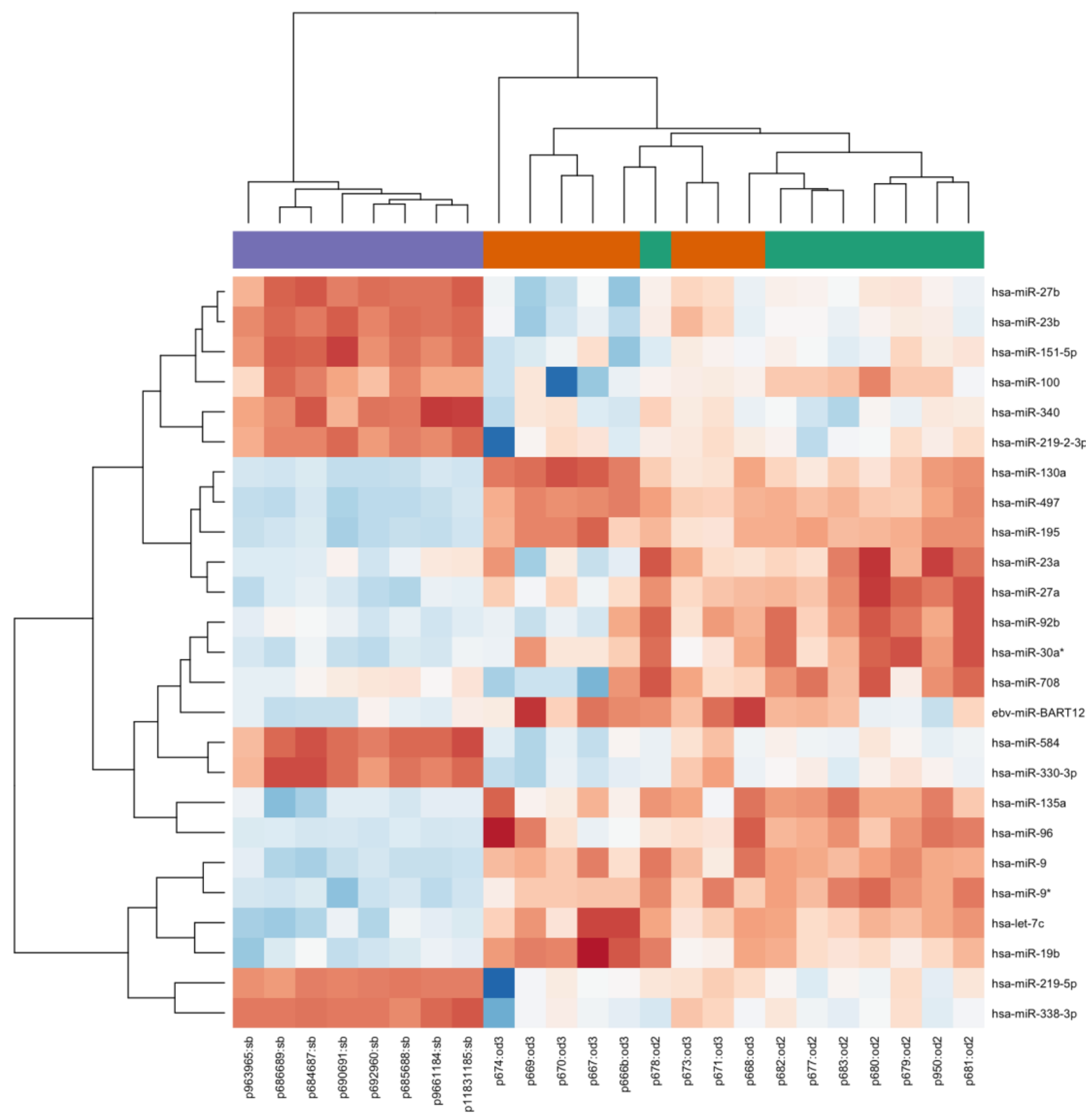

Figure 9: Heatmap showing miRNAs differentially expressed between 3 groups (od2, od 3 and sb). Note that sample 678 (OD2 with atypical areas) has the miRNA profile more related to OD3. od2: grade II oligodendrogliomas; od3: grade III oligodendrogliomas; sb: substance branca i.e. non neoplastic white matter. The colours green, brown and indigo on the top represent grade II ODs, grade III ODs and nnWM respectively. 


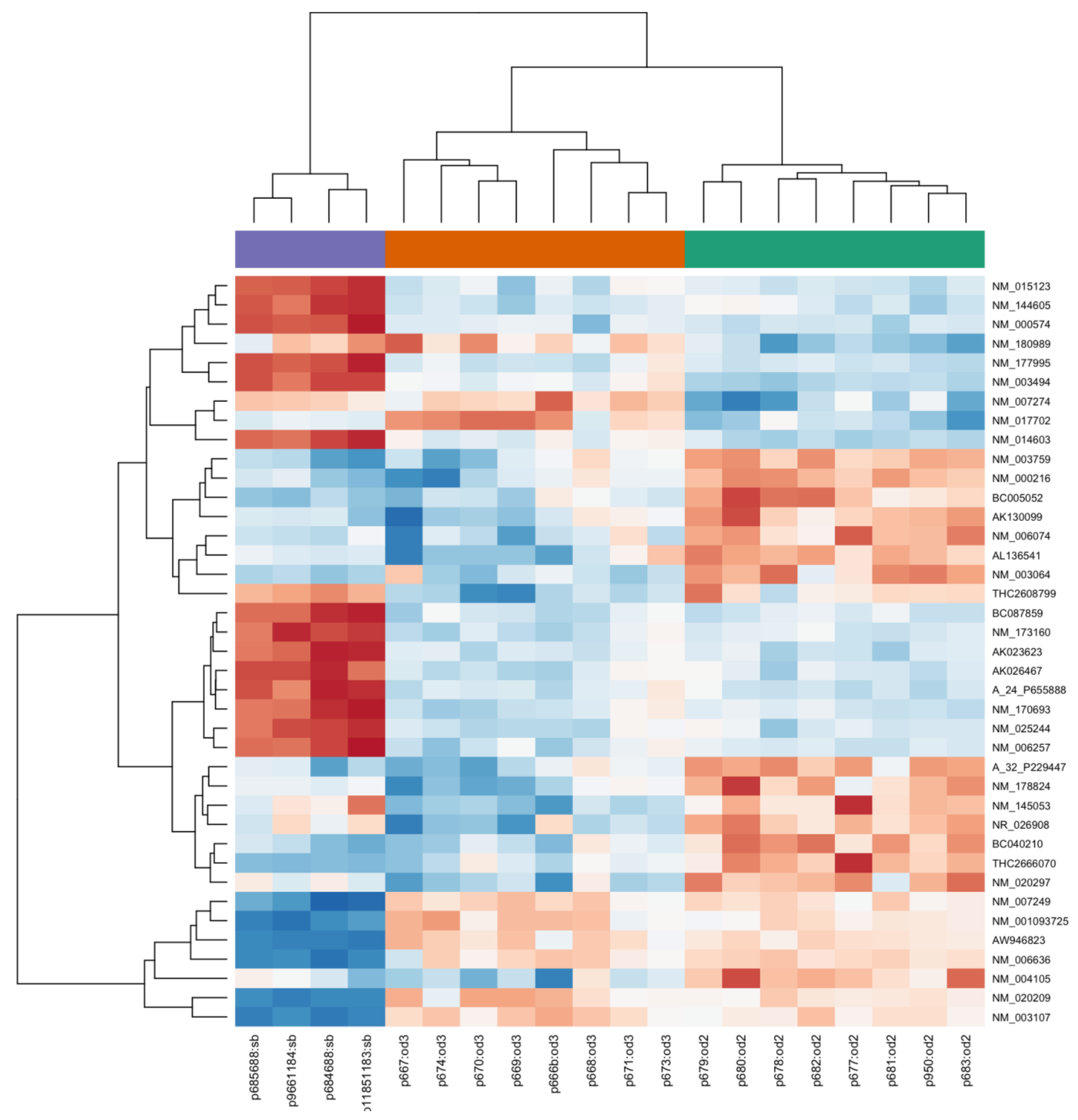

Figure 10: Heatmap showing genes/transcripts differentially expressed between 3 groups (od2, od3 and sb. Note that the sample 678 (OD2 with atypical areas) has gene profile related to OD2. od2: grade II oligodendrogliomas; od3: grade III oligodendrogliomas; sb: substance branca i.e. non neoplastic white matter. The colours green, brown and indigo on the top represent grade II ODs, grade III ODs and nnWM respectively. 


\subsection{Mean miRNAs and mRNAs variations according the tumour grades}

To view the mean difference of expression of both miRNA and mRNA, the set of linear models for expression was performed at the group level (Figure 11 and 12). The 95\% confidence interval was also drawn as presented in the bar graphs below. In these graphs, the points represent the mean expression of the group and the bars indicate the points associated with $95 \%$ confidence interval. Functions of top expressed transcripts (in grade II and III) are given in Table 7, curated from GenBank NCBI with accession numbers from our results.

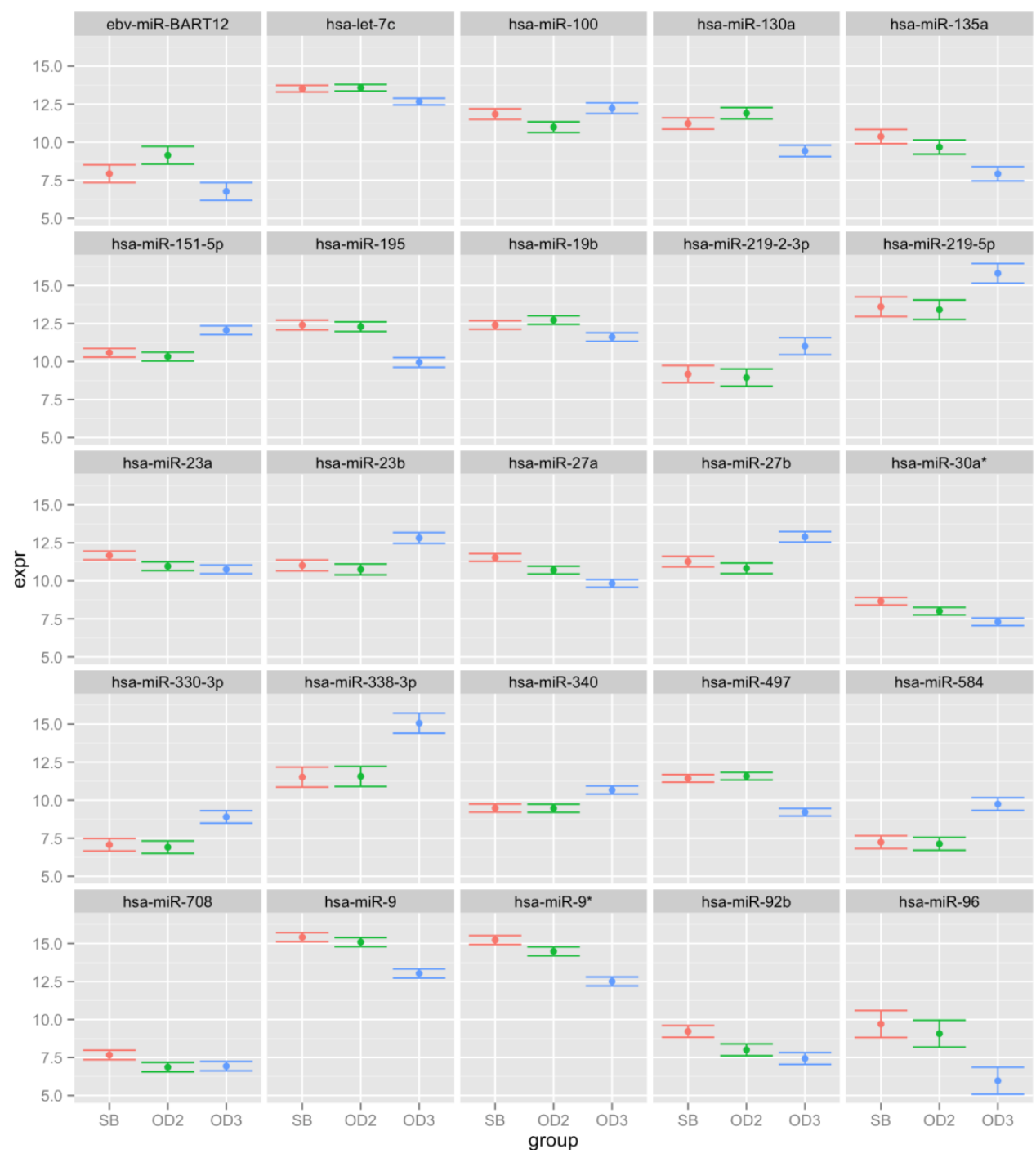

Figure 11: Bar graphs showing the mean miRNAs expression variation according the tumour grade (OD2 green bars and OD3 blue bars compared to nnWM (sb) red bars. OD2: grade II oligodendrogliomas; OD3: grade III oligodendrogliomas; nnWM: non neoplastic white matter. 


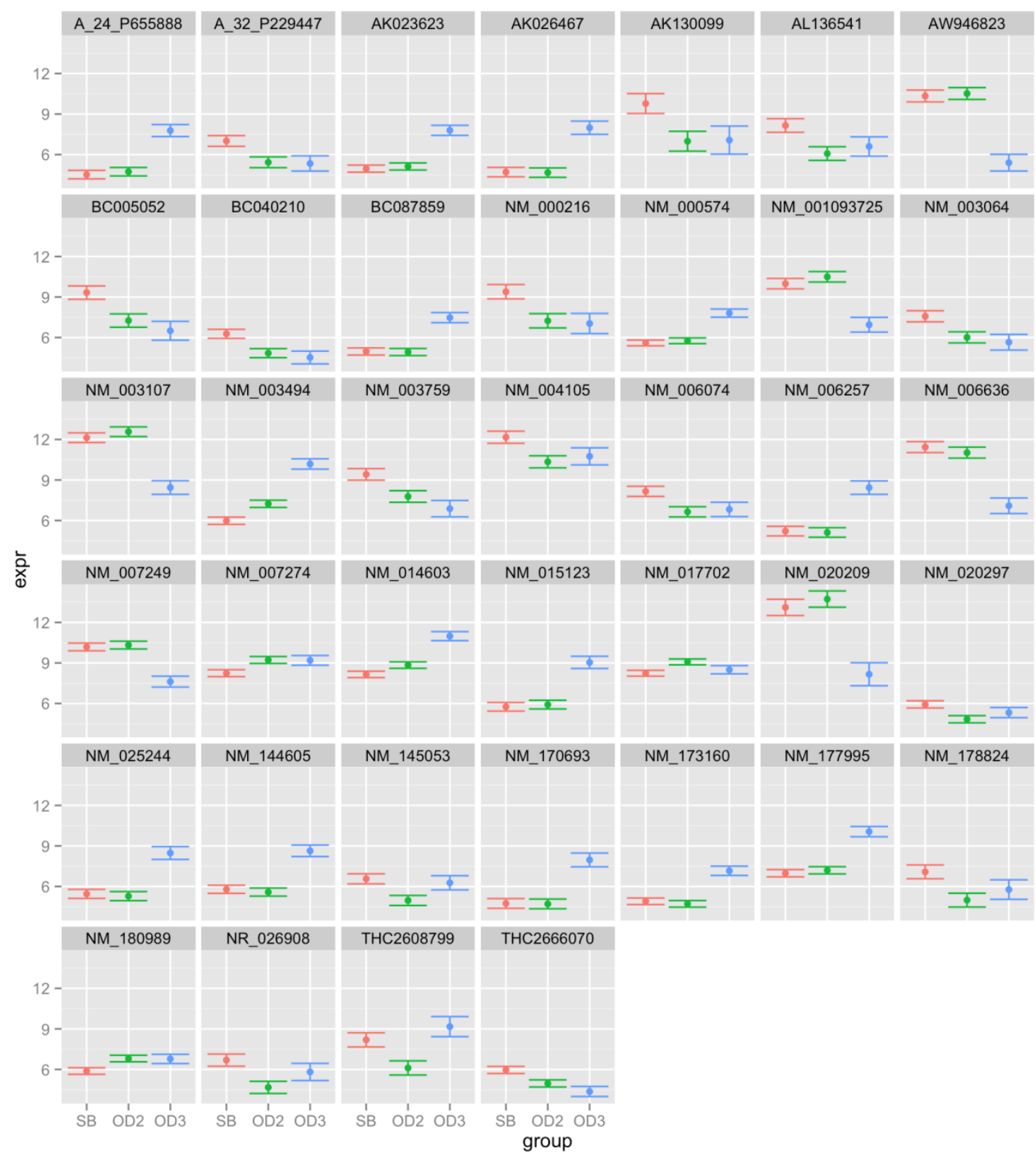

Figure 12: Bar graphs showing the mean mRNAs expression variation according the tumour grade (OD2 green bars and OD3 blue bars compared to nnWM (sb) red bars. OD2: grade II oligodendrogliomas; OD3: grade III oligodendrogliomas; nnWM: non neoplastic white matter. Notably, the grade III has wider range. 
Table 7. Functions of top expressed genes (curated from GenBank NCBI)

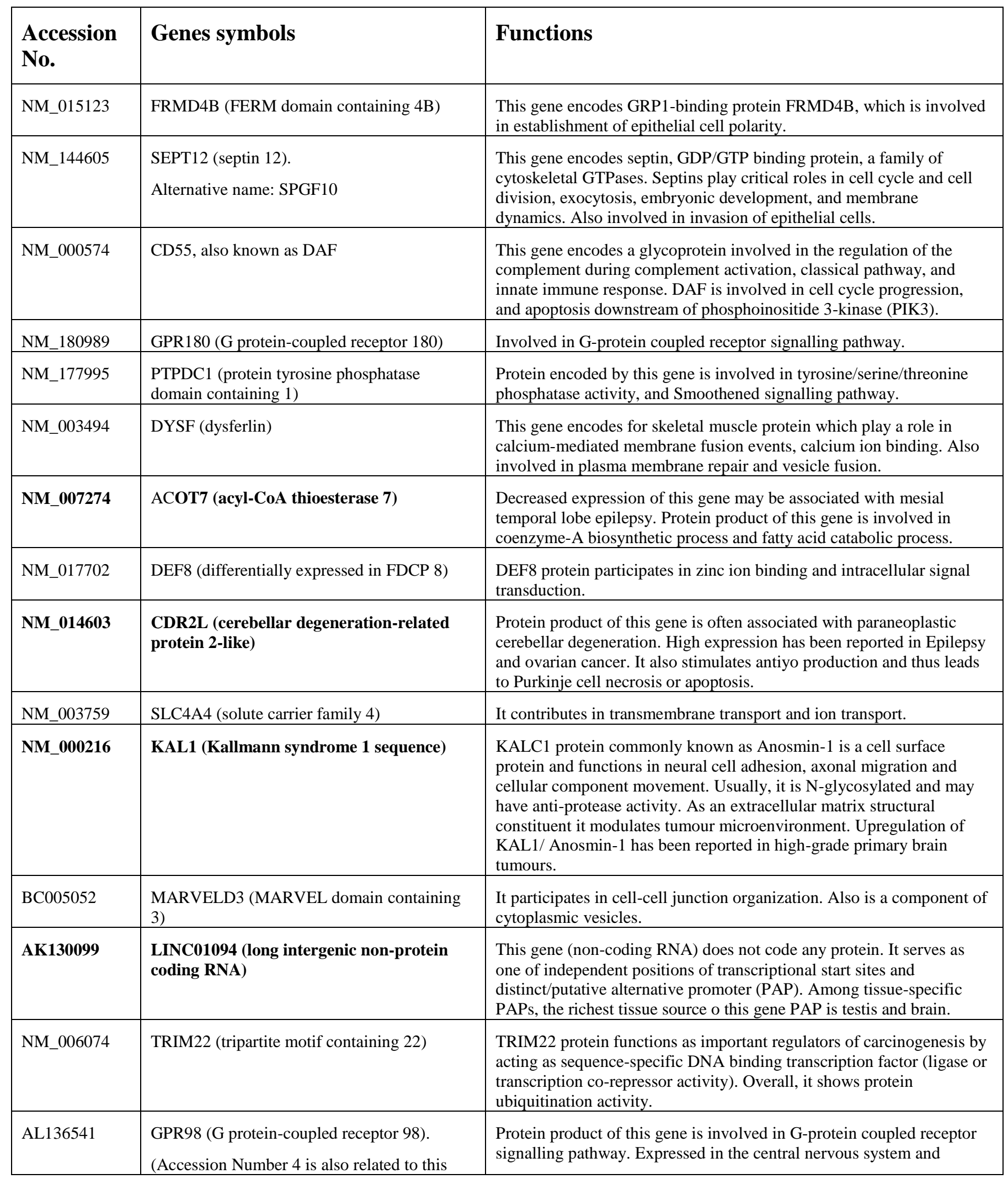




\begin{tabular}{|c|c|c|}
\hline & gene). & involved in nervous system development. \\
\hline NM_003064 & $\begin{array}{l}\text { SLPI (secretory leukocyte peptidase } \\
\text { inhibitor) }\end{array}$ & $\begin{array}{l}\text { Inhibitor type of secreted protein which protects epithelial tissues from } \\
\text { serine proteases. Down regulation or absence of this inhibitor causes } \\
\text { defects in cell cycle resulting in proliferation and have been reported in } \\
\text { carcinogenesis of glioblastoma. }\end{array}$ \\
\hline THC2608799 & unknown & Unknown \\
\hline BC087859 & LOC401317 & Uncharacterized \\
\hline AK023623 & GLDN (gliomedin) & $\begin{array}{l}\text { Protein product of this gene is involved in heterotypic cell-cell } \\
\text { adhesion. Clustering of voltage-gated sodium channels during Schwann } \\
\text { cell-axon interaction. }\end{array}$ \\
\hline A_24_P655888 & unknown & Unknown \\
\hline NM_170693 & $\begin{array}{l}\text { SGK2 (serum/glucocorticoid regulated kinase } \\
\text { 2) }\end{array}$ & $\begin{array}{l}\text { Participates in PI3K-Akt signalling pathway and FoxO signalling } \\
\text { pathway. }\end{array}$ \\
\hline NM_025244 & TSGA10 & $\begin{array}{l}\text { TSGA10 is specifically expressed in astrocytes, whereas } \\
\text { overexpression is associated with progression of brain tumours. } \\
\text { Overexpression is observed in more than } 83 \% \text { of brain tumours. }\end{array}$ \\
\hline NM_006257 & $\begin{array}{l}\text { PRKCQ (protein kinase } \mathrm{C} \text { theta) also known } \\
\text { as PRKCT; nPKC-theta }\end{array}$ & $\begin{array}{l}\text { It shows serine- and threonine-specific protein kinase activity and } \\
\text { phosphorylates variety of protein targets. Also contributes in B/T cell } \\
\text { receptor signalling pathway, and apoptotic cleavage of cellular proteins } \\
\text { during apoptotic execution phase. It also serves as major receptors for } \\
\text { phorbol esters (a class of tumour promoters). PKRC have been reported } \\
\text { in glioma growth through cell cycle regulation when stimulated with } \\
\text { phorbol. }\end{array}$ \\
\hline NM_178824 & WDR49 (WD repeat domain 49) & $\begin{array}{l}\text { This gene encodes WD40 domain which cover adaptor/regulatory } \\
\text { modules in signal transduction, pre-mRNA processing and cytoskeleton } \\
\text { assembly. }\end{array}$ \\
\hline NM_145053 & UBQLNL (ubiquilin-like) & Involves in pathways of protein processing in endoplasmic reticulum \\
\hline NR_026908 & AQP4-AS1 (AQP4 antisense RNA 1) & $\begin{array}{l}\text { This gene does not encode any protein and serve as non-coding RNA. } \\
\text { This gene was revealed as novel antisense RNAs candidate for drug } \\
\text { refractory epilepsy in patients with mesial temporal lobe epilepsy with } \\
\text { hippocampal sclerosis (MTLE-HS). }\end{array}$ \\
\hline BC040210 & $\begin{array}{l}\text { AKR1C1 (aldo-keto reductase family } 1, \\
\text { member } \mathrm{C} 1 \text { ) }\end{array}$ & $\begin{array}{l}\text { It regulates the metabolism of lipids and lipoproteins. Also contributes } \\
\text { to epithelial cell differentiation. }\end{array}$ \\
\hline THC2666070 & unknown & Unknown \\
\hline NM_020297 & ABCC9 (ATP-binding cassette, sub-family C) & It shows potassium channel regulator activity and signal transduction. \\
\hline NM_007249 & $\begin{array}{l}\text { KLF12 (Kruppel-like factor 12) also known } \\
\text { as AP-2 alpha; AP-2rep; HSPC122 }\end{array}$ & $\begin{array}{l}\text { It shows sequence-specific DNA binding transcription factor activity. } \\
\text { Transcription co-repressor activity i.e. negative/positive regulation of } \\
\text { transcription from RNA polymerase II promoter). Such activity has } \\
\text { been reported in in gliomas. }\end{array}$ \\
\hline NM_00109372 & MEX3A (mex-3 RNA binding family member & It contributes in poly(A) RNA binding and acts as cytoplasmic mRNA \\
\hline
\end{tabular}




\begin{tabular}{|l|l|l|}
\hline 5 & A) also known as RKHD4; MEX-3A. & processing body. \\
\hline AW946823 & unknown & Unknown \\
\hline NM_006636 & MTHFD2 & $\begin{array}{l}\text { It helps magnesium ion binding and exhibits methylenetetrahydrofolate } \\
\text { dehydrogenase (NAD+) activity during metabolism. }\end{array}$ \\
\hline NM_004105 & $\begin{array}{l}\text { EFEMP1 (EGF containing fibulin-like } \\
\text { extracellular matrix protein 1) }\end{array}$ & $\begin{array}{l}\text { This gene is upregulated in malignant gliomas and plays important role } \\
\text { in the progression of these tumours. Major activities involve epidermal } \\
\text { growth factor receptor (EGFR) binding and epidermal growth factor } \\
\text { receptor signalling pathway. }\end{array}$ \\
\hline NM_003107 & $\begin{array}{l}\text { SHD (Src homology 2 domain containing } \\
\text { transforming protein D). Commonly cited as }\end{array}$ & $\begin{array}{l}\text { Protein-protein interactions and kinase activity (phosphorylation) and } \\
\text { signal transduction in MAPK pathway). }\end{array}$ \\
\hline SH2 or SHP-2 & $\begin{array}{l}\text { This is intronless gene and encodes a member of the SOX (SRY-related } \\
\text { HMG-box) family of transcription factors involved in the regulation of } \\
\text { cell differentiation and apoptosis pathway. Major functions include as } \\
\text { negative regulation of apoptosis, DNA damage response and signal } \\
\text { transduction by p53 class mediator (which results in cell cycle arrest). } \\
\text { Canonical Wnt signalling pathway. Glial cell development and } \\
\text { proliferation. }\end{array}$ \\
\hline
\end{tabular}

\section{Note:}

- Bold genes are of particular interest and relevance with glioma progression.

- LINC01094 is long intergenic non-protein coding RNA

- AQP4-AS1 is non-coding RNA and acts as antisense RNA 


\subsection{Correlation between miRNAs and their mRNA-targets}

When analysing the possible relationships between miRNAs with their respective mRNA targets, we found that only 4 of the 15 miRNAs upregulated in ODs grade III had their target mRNAs "suppressed", totalling to 868 potential regulation relationships. MiRNA with significant relative increased expression was hsa-miR-301b (Table 8).

Table 8. Overexpressed miRNAs and downregulated mRNAs-targets in grade III anaplastic oligodendrogliomas

\begin{tabular}{|l|l|l|l|}
\hline hsa - miRNA & MIMA Access* & Magnitude & $\mathbf{N}^{\mathbf{0}}$ of mRNA-targets \\
\hline miR-301b & MIMAT0004958 & $4,3 \mathrm{x}$ & 264 \\
\hline miR-130b & MIMAT0000691 & $3,0 \mathrm{x}$ & 253 \\
\hline miR-206 & MIMAT0000462 & $2,6 \mathrm{x}$ & 193 \\
\hline miR-378 & MIMAT0000732 & $1,9 \mathrm{x}$ & 158 \\
\hline & & & Total $=868$ \\
\hline
\end{tabular}

* MIMA: identification of the sequence in miRBase 
20 miRNAs with comparatively decreased expression in grade III AOD had their mRNAs targets, predicted from overexpressed mRNAs in microarray (Table 9), totalling to 2.704 potential relationships of regulation. The miRNA with significant relative lower expression was miR-204 followed by miR-455-5p (Figure 13).

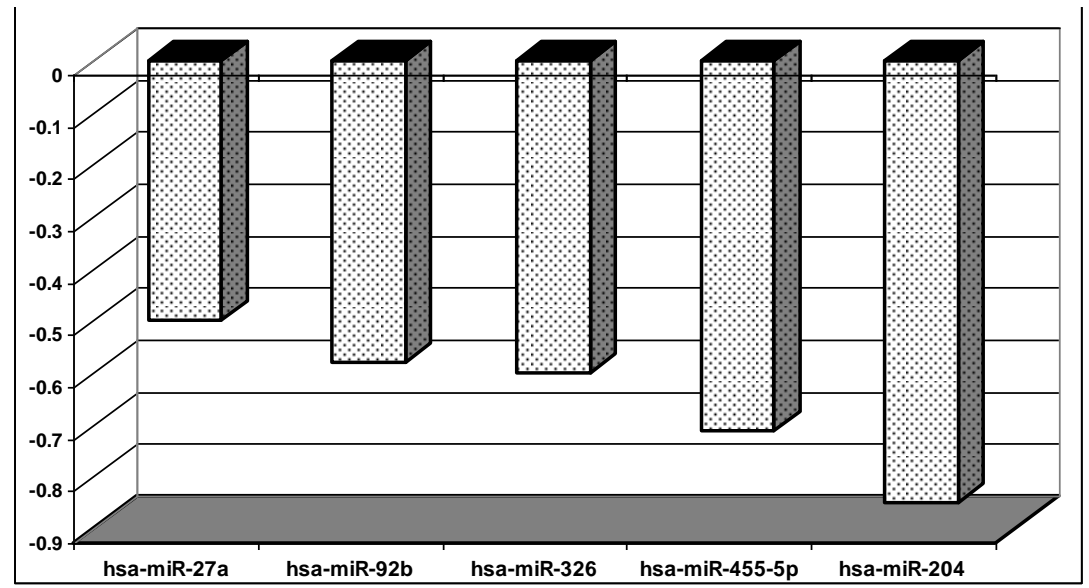

Figure 13: Five miRNAs with relative reduction in expression in grade III anaplastic oligodendrogliomas (AOD) whose mRNAs-targets are reduced 
Table 9. Relative reduction of miRNAs expression in grade III anaplastic oligodendrogliomas

\begin{tabular}{|c|c|c|}
\hline miRNA & Order of Magnitude & Relative Reduction \\
\hline hsa-miR-204 & 0.15 & $-6,60 x$ \\
\hline hsa-miR-455-5p & 0.39 & $-2,56 x$ \\
\hline hsa-miR-326 & 0.40 & $-2,50 x$ \\
\hline hsa-miR-92b & 0.42 & $-2,38 x$ \\
\hline hsa-miR-455-3p & 0.44 & $-2,27 x$ \\
\hline hsa-miR-27a & 0.50 & $-2,00 x$ \\
\hline hsa-miR-22* & 0.54 & $-1,85 x$ \\
\hline hsa-miR-100 & 0.54 & $-1,85 x$ \\
\hline hsa-miR-708 & 0.55 & $-1.81 x$ \\
\hline hsa-miR-23a & 0.56 & $-1.78 x$ \\
\hline hsa-miR-30a* & 0.56 & $-1.78 x$ \\
\hline hsa-miR-181c* & 0.56 & $-1.78 x$ \\
\hline hsa-miR-652 & 0.57 & $-1.75 x$ \\
\hline hsa-miR-99a & 0.57 & $-1.75 x$ \\
\hline hsa-miR-193a-3p & 0.57 & $-1.75 x$ \\
\hline hsa-let-7d* & 0.58 & $-1.72 x$ \\
\hline hsa-miR-152 & 0.59 & $-1.69 x$ \\
\hline hsa-miR-30a & 0.61 & $-1.63 x$ \\
\hline hsa-miR-30c & 0.62 & $-1.61 x$ \\
\hline hsa-miR-29c* & 0.62 & $-1.61 x$ \\
\hline hsa-miR-29c & 0.66 & $-1.51 x$ \\
\hline hsa-miR-421 & 0.67 & $-1.49 x$ \\
\hline hsa-miR-148b & 0.68 & $-1.47 x$ \\
\hline
\end{tabular}




\begin{tabular}{|l|l|l|}
\hline hsa-miR-212 & 0.68 & $-1.47 \mathrm{x}$ \\
\hline hsa-miR-30e* & 0.71 & $-1.40 \mathrm{x}$ \\
\hline hsa-miR-24 & 0.74 & $-1.35 \mathrm{x}$ \\
\hline hsa-miR-770-5p & 0.75 & $-1.33 \mathrm{x}$ \\
\hline hsa-miR-26a & 0.75 & $-1.33 \mathrm{x}$ \\
\hline hsa-miR-22 & 0.77 & $-1.29 \mathrm{x}$ \\
\hline
\end{tabular}

Note: Bold miRNAs did not exhibit the increase of their mRNA in microarray and were subsequently excluded from the analysis. 


\subsection{Upregulated miRNAs and their respective mRNAs downregulated}

Among the various possible relationships between micRNAs/mRNAs in grade III ODs, we chose the following genes whose transcripts were highly expressed: BCL211, NEK1, NEK4, CDK9, CDKL3, BCL-2, CD44, FGF2 and PPAR4 (Tables 10 and 11).

Table 10. Downregulated genes and overexpression of corresponding miRNAs in grade III anaplastic oligodendrogliomas

\begin{tabular}{|c|c|c|c|}
\hline $\begin{array}{l}\text { Gene } \\
\text { (reduced) }\end{array}$ & $\begin{array}{l}\text { GenBank } \\
\text { Access No. }\end{array}$ & $\begin{array}{l}\text { microRNA } \\
\text { (increased) }\end{array}$ & $\operatorname{mirSVR} * * 6$ score ${ }^{9}$ \\
\hline \multirow[t]{2}{*}{ BCL2 } & \multirow[t]{2}{*}{ NM_000633 } & hsa-miR-206 & $-0,183$ \\
\hline & & hsa-miR-301b & $-0,228$ \\
\hline \multirow[b]{3}{*}{ BCL2L11 } & \multirow[b]{3}{*}{ NM_138621 } & hsa-miR-130b & $-0,609$ \\
\hline & & hsa-miR-301b & $-0,616$ \\
\hline & & hsa-miR-301b & $-0,636$ \\
\hline \multirow[b]{6}{*}{ NEK1 } & \multirow[b]{6}{*}{ NM_012224 } & hsa-miR-130b & $-0,944$ \\
\hline & & hsa-miR-378 & $-0,670$ \\
\hline & & hsa-miR-130b* & $-0,944$ \\
\hline & & hsa-miR-378* & $-0,670$ \\
\hline & & hsa-miR-130b* & $-0,944$ \\
\hline & & hsa-miR-378* & $-0,670$ \\
\hline NEK4 & NM_003157 & hsa-miR-378 & $-0,878$ \\
\hline
\end{tabular}




\begin{tabular}{|l|l|l|l|}
\hline FGF2 & NM_002006 & hsa-miR-378 & $\mathbf{- 0 , 1 7 6}$ \\
\hline CDK9 & NM_001261 & hsa-miR-206 & $-0,225$ \\
\hline CD44 & NM_000610 & hsa-miR-130b & $\mathbf{- 0 , 5 1 4}$ \\
\hline CDKL3 & NM_016508 & hsa-miR-206 & $-1,011$ \\
\hline PPP4R4 & NM_058237 & hsa-miR-206 & $\mathbf{- 0 , 1 2 0}$ \\
\hline
\end{tabular}

Note: bold genes that were subjected to validation by RT-qPCR

* Different probes of the same gene in mRNA microarray

** Calculated by analyses tool mirSVR (www.microRNA.org). 
Table 11. Predicted biological roles of downregulated genes against upregulated miRNAs in grade III anaplastic oligodendrogliomas

\begin{tabular}{|l|l|l|}
\hline Gene & Locus & Principal biological role \\
\hline BCL2 & $18 \mathrm{q} 21.3$ & Resistance to apoptosis \\
\hline BCL2L11 & 2q12-q13 & Induction of apoptosis \\
\hline NEK1 & $4 \mathrm{q} 33$ & Control of mitosis (kinase activity) \\
\hline NEK4 & $3 \mathrm{p} 21.1$ & Cell cycle regulation (kinase) \\
\hline CDK9 & $9 \mathrm{q} 34.1$ & Cell cycle regulation (kinase) \\
\hline CDKL3 & $5 \mathrm{q} 31$ & Modulation of cell cycle and proliferation \\
\hline CD44 & $11 \mathrm{p} 13$ & Cell adhesion and signalling \\
\hline FGF2 & $14 \mathrm{q} 25-\mathrm{q} 27$ & Angiogenesis, neurogenesis and proliferation \\
\hline PPP4R4 & $14 \mathrm{q} 32.2$ & Cell interaction \\
\hline
\end{tabular}

Note: bold genes that were subjected to validation by RT-qPCR 


\subsection{Downregulated miRNAs and their respective mRNAs upregulated}

Similarly, we selected the following upregulated genes (and their miRNAs downregulated): PDGFA, RAB26, PDPK1, HDAC2, CCND1, DVL2, RAD54B, FADD, PGK1 and BRAF

among others. Additionally, we submitted a list of transcripts $(n=645)$ by DAVID bioinformatics analysis tool, obtaining few miRNAs and their respective transcript-targets, such as hsa-miR-23a / BRAF-CCND1, hsa-miR-24 / IGFBP4, HDAC2, hsa-miR127a / PDPK1, hsamiR30c / RAB26 and hsa-miR-193a-3p. These miRNAs are related to the regulation of proliferation / differentiation and embryonic stem cell differentiation, described previously [50], and various physiological and pathological processes [129]. For this reason we have chosen these transcripts to be validated by RT-qPCR.

\subsection{Validation of miRNAs and mRNAs by RT-qPCR}

Among miRNAs and the genes chosen for validation of the data obtained for confirmation of microarray transcribed in Tables 12 and 13 (functional relevance of validated miRNAs and their mRNAs is described in discussion section). 
Table 12. Summary of the results of microarray data and RT-qPCR validation of relatively downregulated miRNAs and their mRNAs targets in grade III ODs

\begin{tabular}{|l|l|l|l|l|}
\hline hsa-microRNA & miRNA-array & RT-qPCR & $\begin{array}{l}\text { mRNA-array } \\
(\text { increased })\end{array}$ & RT-qPCR \\
\hline miR-193a-3p & Reduced & $\begin{array}{l}\text { Validated } \\
(\mathrm{p}<0.05)\end{array}$ & DVL2 & $\begin{array}{l}\text { not validated } \\
(\mathrm{p}>0.05)\end{array}$ \\
\hline miR-23a & Reduced & not validated ( $>0.05)$ & CCND1 & $\begin{array}{l}\text { Validated } \\
(\mathrm{p}<0.05)\end{array}$ \\
\hline miR-24 & Reduced & $\begin{array}{l}\text { Validated } \\
(\mathrm{p}<0.05)\end{array}$ & HDAC2 & $\begin{array}{l}\text { Validated } \\
(\mathrm{p}<0.05)\end{array}$ \\
\hline miR-27a & Reduced & $\begin{array}{l}\text { Validated } \\
(\mathrm{p}<0.05)\end{array}$ & $\begin{array}{l}\text { PDPK1 } \\
\text { not validated } \\
\text { miR-30a-5p }\end{array}$ \\
\hline miR-30c & Reduced & $\begin{array}{l}\text { Validated } \\
(\mathrm{p}<0.05)\end{array}$ & $\begin{array}{l}\text { Validated } \\
(\mathrm{p}<0.05)\end{array}$ \\
\hline
\end{tabular}

Note: Bold transcripts whose expression level was confirmed by RT-qPCR. 
Table 13. Summary of the results of microarray data and validation by RT-qPCR of miRNAs with relative overexpression and their mRNAs in grade III ODs

\begin{tabular}{|l|l|l|l|l|}
\hline hsa-microRNA & miRNA-array & RT-qPCR & $\begin{array}{l}\text { mRNA-array } \\
\text { (decreased })\end{array}$ & RT-qPCR \\
\hline miR-130b & increased & not validated (p>0.05) & CD44 & $\begin{array}{l}\text { validated } \\
(\mathrm{p}<0.05))\end{array}$ \\
\hline miR-206 & increased & $\begin{array}{l}\text { not validated } \\
(\mathrm{p}>0.05)\end{array}$ & PPAR4 & $\begin{array}{l}\text { validated } \\
(\mathrm{p}<0.05)\end{array}$ \\
\hline miR-301b & increased & $\begin{array}{l}\text { validated } \\
(\mathrm{p}<0.05)\end{array}$ & BCL2 & $\begin{array}{l}\text { validated } \\
(\mathrm{p}<0.05))\end{array}$ \\
\hline miR-378 & increased & $\begin{array}{l}\text { validated } \\
(\mathrm{p}<0.05)\end{array}$ & $\begin{array}{l}\text { FGF2 } \\
(\mathrm{p}<0.05)\end{array}$ \\
\hline
\end{tabular}

Note: Bold transcripts whose expression level was confirmed by RT-qPCR. 


\subsection{Establishment of primary cells lines of oligodendrogliomas}

Since there is no commercial lines and the need to subsequently perform functional studies, we were able, with great difficulty, to establish primary lines (in-house) 2 cases of ODs, 1 line of a grade II and one of grade III (Figures 14 and 15). In both strains, the neoplastic cells showed no immunoreactivity of Gal-3 and GFAP (data not shown). Morphologically speaking there is no specific marker oligodendrogliomas morphology by immunohistochemical analysis. Therefore, Gal-3 was used to analyse ODs morphology, although not exclusive marker
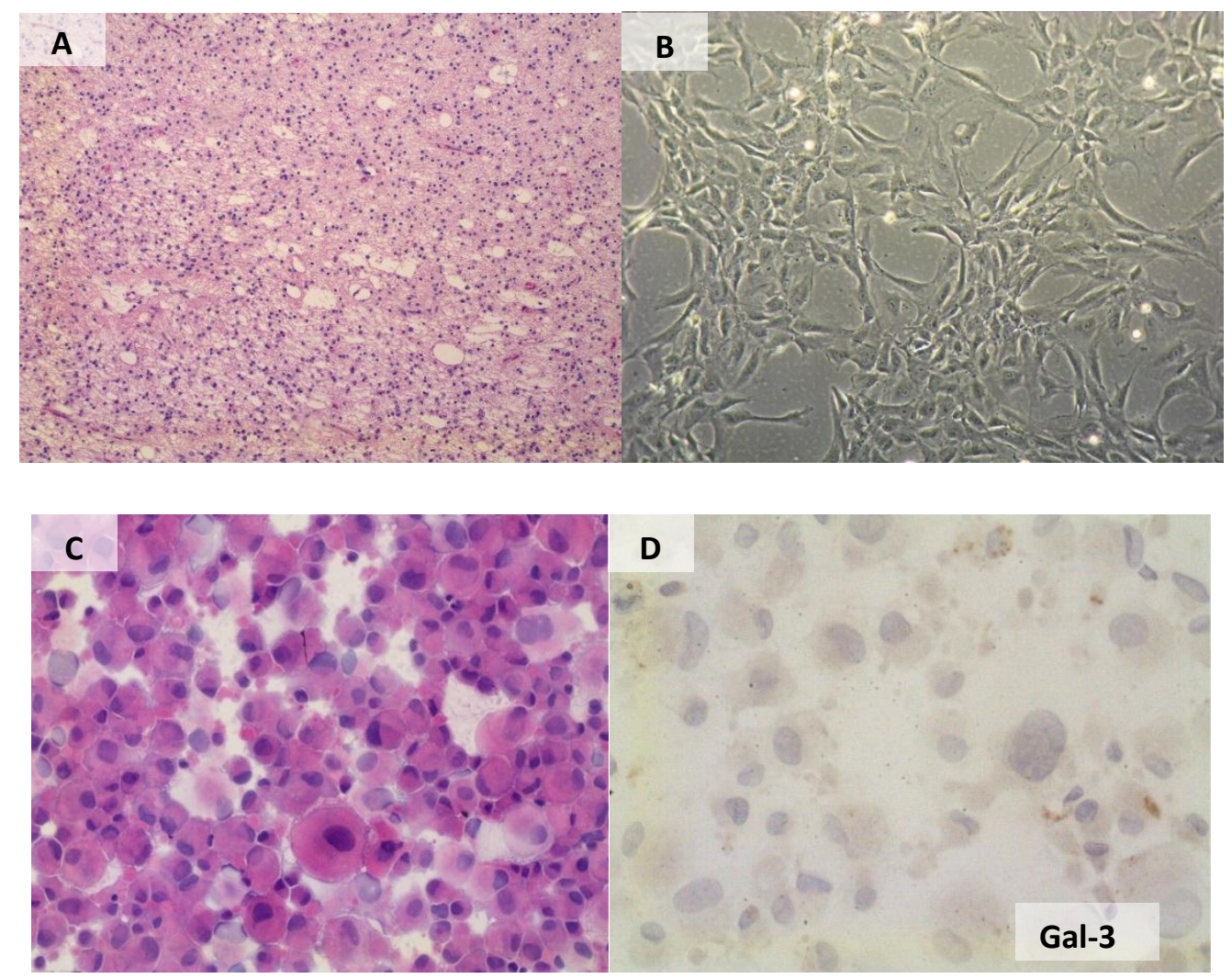

Figure 14: Primary culture of grade II oligodendrogliomas: A- morphological appearance of cancer $(\mathrm{H} \& \mathrm{E})$. (B) Cells in culture. C- Photomicrocrograph the cells after suspension and fixation pellet in $(\mathrm{H} \& \mathrm{E})$. (D) Immunohistochemical reaction for Galectin-3. Note the absence of Gal-3 immunoreactivity in neoplastic cells. 

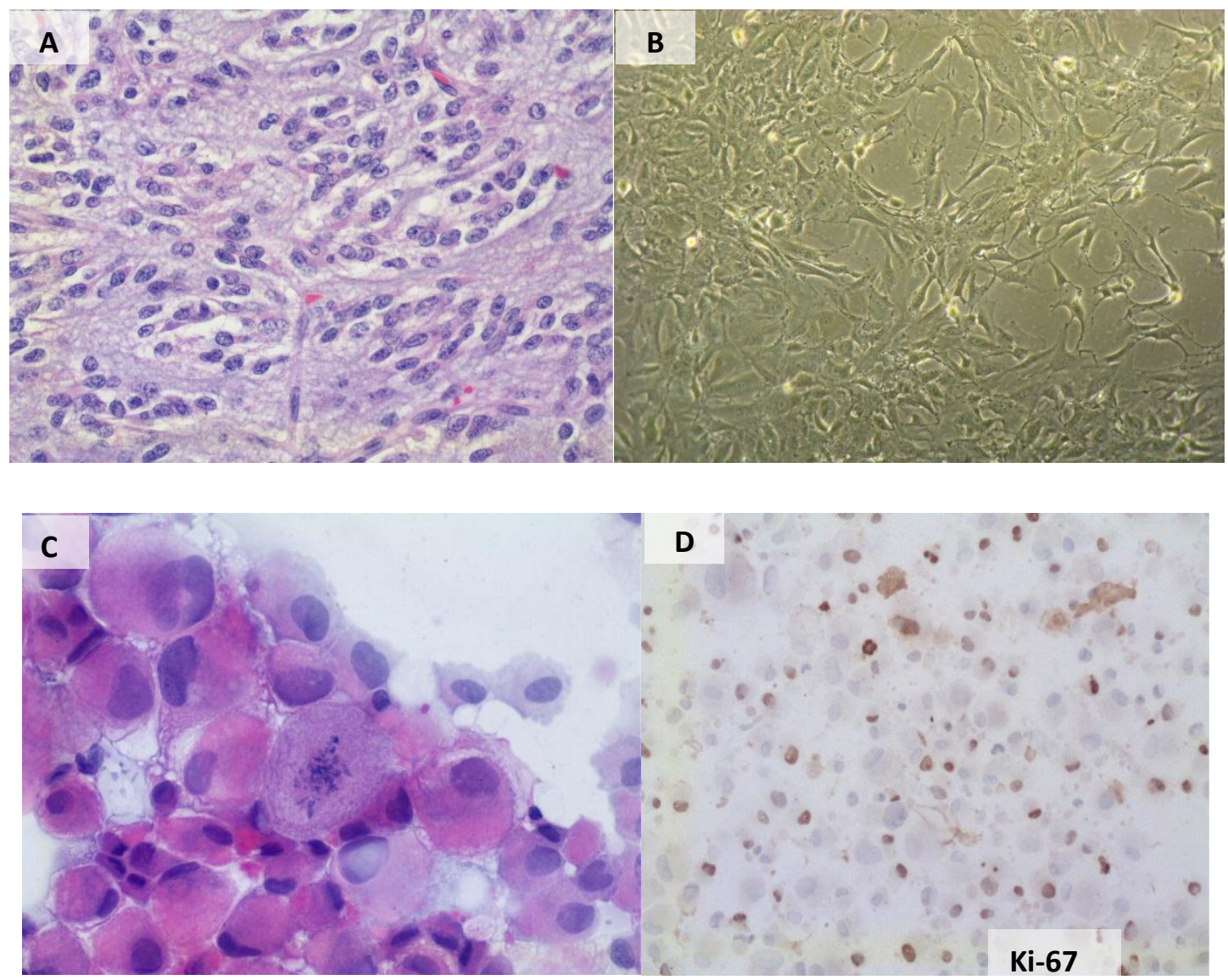

Figure 15: Primary culture of grade III oligodendrogliomas: A- Morphological appearance of cancer (H \& E). (B) Cells in culture. (C) Photomicrocrograph the cells after suspension and fixation pellet in $(\mathrm{H} \& \mathrm{E})$. (D) immunohistochemical reaction for Ki-67. Note the large number of cells in the active phases of the cell cycle. 


\subsection{Validation of miRNAs in cell lines}

The hsa-miR-23a and hsa-miR-27a functions were tested in BJ cells (human adult fibroblasts) by theClick iT® EdU Cell Proliferation Assay and FITC Annexin V apoptosis detection. Cells treated with pre-miR-23a and pre-miR-27a presented a reduction of $20 \%$ on cell proliferation in comparison with controls (data not shown), but no significant results were observed in Annexin $\mathrm{V}$ assays. In this case, the reduction of cell proliferation was apoptosis-independent. 


\section{DISCUSSIONS}

Oligodendrogliomas represent distinct clinical behaviour as compared to astrocytomas accounting for their unusual molecular features and genetic alterations. However, little is known how such genetic alterations correlate with global patterns of miRNA expressions and gene expression in oligodendrogliomas. It is now well appreciated that miRNAs play a central role in tumorigenesis by dynamically regulating gene expression, whereas, their expression profiling provides better insights into tumour biology than traditional histopathological approaches [182, 229, 269]. In this regard, differential expression of miRNA and mRNA between different grades of malignant progression (i.e. grade II and grade III) of oligodendrogliomas could provide better insight into tumour biology as well as can help identifying prognostic signatures in ODs.

In this study, we performed microarray analysis to examine the miRNA and mRNA profiles differentially expressed in anaplastic oligodendroglioma (OD3) and grade II oligodendrogliomas (OD2) in comparison to non-neoplastic white matter (nnWM).

Among all identified candidates for differential expression in three groups (OD2, OD3 and nnWM), almost all patient samples in OD3 showed clear distinction of miRNA and mRNA expression patterns different from nnWM samples. Surprisingly, one of the unusual observation in our results was a distinct miRNA signature from a patient sample 678 (Figure 9), initially identified as OD2, has a miRNA signature matched with OD3 cases. Initially it gave us impression that this might be described as a border line between OD2 and OD3 or might have some unique feature that need to be further investigated. However, this was also suspected that there might be an error during classification of samples. Later on, careful re-evaluation by pathological features revealed that this sample belongs to astrocytoma but not OD2. However, miRNA profile of this patient sample was closer to OD3.

In our cases we identified unique sets of miRNAs as well as mRNAs that are differentially expressed between OD3 and OD2 indicating grade specific expressions. Among differentially expressed miRNAs, several are downregulated in grade III ODs and their target mRNAs are upregulated that are implicated in cell proliferation and are associated with higher degree of malignancy. For example, 25 potential miRNAs showed expression variation according the 
tumour grade. Among them miR-23a, miR-27a, miR-130a, miR-96a are most relevant to grade III ODs.

The miR-23a is downregulated in our cases of OD3 which targets the BRAF (v-raf murine sarcoma viral oncogene homolog B1). The BRAF gene is up regulated in OD3 which is responsible for encoding a protein family of kinases (MAPkinase/ERKs). Characteristically, tandem duplication and fusion of BRAF with KIAA1549 are seen in pilocytic astrocytomas, can aid in diagnosis and possibly the therapeutics [270]. Mutations in BRAF have recently been reported in anaplastic oligodendrogliomas [20]. In our cases, the relative overexpression of BRAF in anaplastic oligodendrogliomas (OD3) associated with cell proliferation and loss of cell differentiation is greater than those observed in low-grade oligodendrogliomas (OD2). In contrast, some miRNAs are up regulated and their target genes are downregulated that previously were implicated in cell cycle control, cell adhesion and apoptosis. Of particular note, the expression variation is grade dependent that could serve a prognostic value.

We were able to validate 10 key miRNAs and their respective gene targets by RT-qPCR potentially involved in the malignant progression of oligodendrogliomas. Two pools of miRNAgene differential expression were confirmed in OD3 ( $<<0.05)$ : First pool, repressed miRNAs/upregulated genes and other overexpressed miRNAs/downregulated genes. In the first pool, 5 miRNAs including miR-193a-3p, miR-24, miR-27a, miR-30a-5p and miR-30c were suppressed whose target genes such as CCND1, HDAC2, PDGFA, and RAB-26 were upregulated (increased levels of mRNAs).

Among the upregulated genes, the CCND1 gene (regulated by miR-23a) is cyclind D1 also known as BCL1 or PRAD1 is a cell cycle regulator. This protein has been shown to interact with tumour suppressor protein $\mathrm{Rb}$ and the expression of this gene is regulated positively by $\mathrm{Rb}$. Overexpression of this gene, which alters cell cycle progression, is observed frequently in a variety of tumours and may contribute to tumorigenesis. Recently it has been reported in brain tumours [271, 272]. Interestingly, the cell proliferative assay of hsa-miR-23a and hsa-miR-27a validated in human adult fibroblasts exhibited $20 \%$ reduction in cell proliferation (independent of apoptosis). This confirms that target genes have role in cell cycle regulation. 
HDAC2 gene (regulated by miR-24) is histone deacetylase 2, which forms a transcriptional repressor complex and plays an important role in transcriptional regulation, cell cycle progression and developmental events. Recently it has been reported as negative regulator of apoptosis [273]. PDGFA gene (regulated by miR-30a-5p) is platelet-derived growth factor alpha involved in cell proliferation and motility [274], and has been reported to modulate glioma microenvironment [275]. Majority of astrocytoma and oligodendrogliomas exhibit amplification of PDGF and genes encoding their ligands [66, 68, 69, 71]. RAB-26 gene (regulated by miR$30 \mathrm{c}$ ) is a member of RAB protein family that regulates vesicle fusion and intercellular vesicle trafficking, endocytosis, exocytosis, and recycling [276, 277].

The second pool was comprised of overexpressed miRNAs/downregulated genes, which include BCL2 (regulated by miR-301b), FGF2 (miR-378), CD44 (miR-130b) and PPP4R4 (miR-206). It has been shown that the $\mathrm{Bcl} 2$ protein has anti-apoptotic function [278-280]. BCL2L11 reduction of expression has been described in up to $40 \%$ of cases of colorectal cancer [278, 281]. It is of interest that invasive glioma cells reduce transcription of anti-apoptotic genes [282]. The suppression of $\mathrm{Bcl} 2$ by overexpression miRNAs was first time reported in chronic lymphocytic leukaemia showing that miR-15a and miR-16-1 overexpression suppress Bcl2 expression [283]. In a somewhat similar way, we could speculate that overexpression of miR-301b could suppress the expression of BCL-2 gene and thus less production of $\mathrm{Bcl} 2$ protein that would be related to the increased apoptosis in anaplastic oligodendrogliomas. In contrast, other target of the miRNA is BCL2L11 gene which is inducer of apoptosis. The protein encoded by BCL2L field 11 contains a BH3 (Bcl-2 homology domain 3), which interacts with other members of the BCL-2 family, including Bcl-2 protein itself, bcl-2L1 and MCL1, and inactivates them [279].

Another gene FGF2 (regulated by miR-378), is expressed in large quantities in normal brain tissue and the pituitary gland. Protein encoded by this gene belongs to fibroblast growth factor (FGF) family having mitogenic and angiogenic activities [284, 285]. The FGF2 has been related to the differentiation of pre-oligodendrocyte progenitor cells from embryonic stem cells of the human species. Expression of this gene inhibits differentiation of progenitor cells into preoligodendrocyte progenitor [286]. However, in our case it is apparent that miR-378 through downregulating FGF2 could have inhibitory effects. 
Among downregulated genes, 4 genes are related to cell cycle control and include NEK1, NEK4, CDK9 and CDKL3. The NEK1 presents involved in the initial response to DNA damage by ionizing radiation and DNA repair. PPPAR4 (regulated by miR-206) and CD44 (regulated by miR-130b) are related to cell-cell interactions, cell adhesion and migration [287-289]. Importance of the expression of PPAR4 family genes has been described in the differentiation of normal and neoplastic cells and the maturation of oligodendrocytes [290]. However, in the present study we observed a reduced expression in PPPAR4 gene in OD3.

Utilizing gene expression data from samples of different grades of OD, it is possible that the mere knowledge of physical interactions between specific miRNAs and mRNAs can be used to determine associated regulatory interactions, involving putative miRNAs that potentially modulate the expression of mRNAs. Despite progress in the determination of miRNA-mRNA interactions, their regulatory role in cancer is only beginning to be unravelled, single miRNA can modulate hundreds of different target mRNAs, and / or that mRNA can be regulated by single or multiple miRNAs - which may explain some of the inconsistencies in the validation of certain miRNAs and mRNAs. The different expression pattern observed in tumours at different stages of malignancy is probably dependent on the cell-specific repertoire of target genes of tumours, sharing different molecular pathways activity. In addition, the participation of pseudogenes in the regulation of gene expression has recently been described, along with the miRNAs [291, 292].

\section{CONCLUSIONS}

Given that ODs exhibit histological malignant transformation accompanying events such as distinctive genetic and epigenetic profile with marked mutational heterogeneity; such genetic entities may offer more restrictive diagnosis of oligodendroglial features and may help appropriate decision to therapy.

The present study does at least provide transcriptional insights into oligodendrogliomas biology and may assist in finding new diagnostic and therapeutic tools for these tumour subsets of different degrades of malignancy (OD grade II, AOD grade III). Summarizing our data, of

particular importance are the miRNAs whose mRNAs-targets are relevant in the context of cancer progression particularly anaplastic oligodendrogliomas (AOD, grade III). Down regulated miRNAs with their target mRNAs overexpression and upregulated miRNAs with their target 
mRNAs suppressed in AODs will be of particular interest. This implies that, deregulated miRNA-mRNA interactions are critical to malignant progression of anaplastic oligodendrogliomas which in our study were attributed to differentiation and neurogenesis, cell cycle regulation, resistance to apoptosis, cell proliferation, cell adhesion, and angiogenesis.

Given the critical need for therapies that should effectively target the invasive cell component of glioma, these data suggest that selective modulation of specific miRNA:mRNA expressions may provide a rich area for therapeutic development to improve clinical outcome in ODs. The knowledge from miRNAs-genes interaction in anaplastic ODs (AOD) reveals potential unique targets that could be used as therapeutic targets for inhibiting the invasive cell population. Using this knowledge, miRNA mimics and inhibitors could be constructed to modulate the mRNA targets thus serving as potential anti-tumour candidates. However, future studies will warrant the validation of such candidates in order to target malignant progression of ODs in particular grade III AOD. The studies will also be needed to observe treatment response using transcriptome profiles to realize their prognostic value.

\subsection{Future perspectives}

As there are contradictive reports that the 2016-WHO defined genetic entities in some cases do not circumscribe with morphological features as proposed by WHO system [40]; therefore, WHO-2016 system needs to be validated rigorously for analysing defined genetic entities concordance with phenotypic/pathologic analysis. While considering ODs; the segmental chromosomal loss of $1 \mathrm{p}$ and 19q by fluorescence in situ hybridization (FISH) [293, 294], may contribute to diagnosis and prognosis of ODs in accordance with WHO-2016 guidelines. In pertinent to examining segmental chromosomal losses by FISH and NGS, it has been proposed that NGS is superior to FISH in distinguishing segmental chromosomal losses from whole-arm deletions [295]. Therefore a single, targeted NGS assay may serve as the sole testing tool for categorizing ODs and other lower grade diffuse gliomas in compliance with the WHO-2016 diagnostic scheme.

Since $1 \mathrm{p} / 19 \mathrm{q}$ co-deletion is the potential hallmark of ODs, in future, of particular interest will be those miRNAs that are mapped to chromosome $1 \mathrm{p}$ and $19 \mathrm{q}$ and are under-expressed. Moreover, the orthogonal intersection of such molecular characteristics (transcriptome pattern in our case) 
with existing genetic markers, such as IDH1 mutations and $1 \mathrm{p} 19 \mathrm{q}$ status may compliment accurate and reliable pathological diagnosis ODs. The universal expression patterns and detailed transcriptome to dissect glioma subtypes needs to be investigated using more advanced techniques, such as higher capacity microarrays, next-generation RNA-sequencing (RNA-Seq) and FISH analysis. Single-cell transcriptome analysis may help to understand genetic influence/genotype (mutations and chromosomal aberrations within tumour sub-clones) and nongenetic influences/phenotype (characterized by progenitor-like tumour cells with differentiated glial subpopulations of tumour cells and 2 dominant subpopulations of tumour cells) [296]. In fact, single-cell RNA-seq supports a developmental hierarchy in human ODs (i.e. support the cancer stem cell model); therefore, profiling single cells from IDH mutant ODs by high throughput RNA-seq may help to reconstruct their developmental programs from genome-wide expression signatures [297]. In addition to genetic influences (genotype) and non-genetic influences (phenotype) of malignant cells, the tumour subclasses differ according to composition of the tumour microenvironment.

Examining genetic influences in IDH-mutant ODs (astrocytic and anaplastic) by combining single-cell RNA-seq profiles from patient samples may provide general framework for dissecting the differences among human tumour subclasses [298]. Although both tumour types share similar developmental hierarchies and lineages of glial differentiation, however differences in bulk profiles between IDH-mutant astrocytoma and OD can be primarily explained by distinct tumour microenvironment and signature genetic events. In light with these arguments, decoupling genetic influences, lineages/subpopulations, and microenvironment in IDH-mutant gliomas by single-cell analysis may help strict diagnosis and therapeutic decisions with substantial implications for disease management.

Finally, integrative analysis of genetic influences such as mutations, genotypic heterogeneity, retrospective analysis of sequencing results, and methylation patterns with non-genetic influences such as phenotypes, decoupling lineages of cellular populations (two muationally different clones) and their interaction with tumour microenvironment (different secreted growth factors) with implementation of high-throughput technology ultimately will dissect molecular classes of ODs that are prognostically significant, and may thus complement the diagnosis, prognosis and patient management. 


\section{CHAPTER II}

RNA and Protein Analysis in Exosome Vesicles

University of Gothenburg

Institute of Medicine, Sahlgrenska Academy,

Department of Rheumatology and Inflammation Research

(Sweden) 


\section{ABSTRACT}

Exosomes are nanosized vesicles, secreted from almost every cell type studied so far. Although several other types of vesicles are reported, however exosomes are the best characterized as compared to other populations of secreted vesicles. Exosomes originate from endosomes, matured in multivesicular bodies (MVBs) of the late endosomes and are finally secreted from cell through exocytosis. During this process, a variety of bioactive molecules are incorporated into exosomes. These include proteins and nucleic acids such as DNA, mRNAs, and non-coding RNAs (ncRNAs) including microRNAs (miRNAs). However, mechanisms by which these molecules are packaged into exosomes remain largely unclear. Exosomes transport genetic material between cells, acting as novel means of intercellular communication. Albeit, the exosome secretion is ubiquitous process, their secretion under pathological conditions is altered, and the content of exosomes differs under such conditions as compared to normal ones.

Since, RNAs are found in exosomes we aimed to study the mechanisms of RNA packaging into exosomes and report few potential RNA-binding proteins that are responsible for RNA packaging into exosomes. Moreover, based on the fact that exosomes naturally transport genetic material between cells, we aimed to engineer exosomes for their utility as gene delivery vectors (GDVs) and evaluated their potential in gene therapy. We were able to develop a novel method for using exosomes as GDVs with enhanced delivery efficacy (results are protected).

Finally, based on the fact that exosomes contain differentially expressed contents under pathological conditions, we studied the protein and ncRNA content of exosomes secreted from cancer cells to examine if there could be potential biomarkers for cancer. Several RNA and protein molecules were identified from lung cancer and breast cancer cell lines; some were identified as potential candidate biomarkers of cancer. To further understand their biological functions, the network analysis and pathway analysis was performed by bioinformatics tools which revealed their participation in cell signalling and cell proliferation. In summary, our results showed that there exist unique mechanisms of RNA packaging into exosomes. Exosomes can be used for gene delivery and can be harvested for biomarker discovery as well as their role in disease progression. These small vesicles have enormous potential in nano-medicine.

Keywords: Exosomes, Nano vesicles, ncRNA, microRNA, Proteins, RNA packaging into exosomes, Gene delivery vectors, Biomarkers 


\section{INTRODUCTION}

\subsection{Exosomes}

Exosomes are nano-sized vesicles (40-150 $\mathrm{nm}$ in size) secreted by many cell types, initially reported from reticulocytes [299-301], and antigen presenting cells (APCs) such as B lymphocytes, dendritic cells and mast cells [302-304]. Exosomes can also be secreted from microglia and neurons [305-307], muscle cells [308], adipocytes [309], malignant effusions and tumour cells [277, 310, 311], and stem cells [312,313]. The process of exosome secretion seems to be ubiquitous and evolutionarily conserved across the species cross-kingdoms [314], ranging from prokaryotes to eukaryotes - including plants [315-317], fungi [318-320], bacteria [321323], viruses [324-327], and parasitic protozoa such as leishmania and malarial parasites [328332] among others.

The exosomes are secreted under both physiological and pathological conditions and are implicated in transport of biological material between cells and serve as mediators of cell-to-cell signalling and intercellular communication. Exosomes secreted from various organs may travel to long distance in the body through biological fluids and are widely distributed in blood plasma and serum [333, 334], urine [277, 335], saliva and breast milk [336-339], cerebrospinal fluid [340], bronchoalveolar fluid [341, 342], synovial fluid [343], amniotic fluid [344], epididymal fluid and seminal fluid [189, 345]. Moreover, exosomes could be secreted into supernatant of cell cultures.

The currently known exosomes have long been considered as platelet-derived particles and were described as cellular dust or debris unless having recognized as exosomes by Johnstone et al., in 1987 [301]. However, studies from Raposo and colleagues [302], Zitvogel and colleagues [303], and later own Théry et al., [304], initially described the functional importance of exosomes in immunological responses and many recent studies have highlighted their potential in pathophysiology, their utility as biomarker discovery and therapeutic potential (such functions will be discussed in later sections). 


\subsubsection{Biogenesis and secretion of exosomes}

Albeit, the process of exosome secretion seems to be evolutionary conserved, however the mechanisms of their biogenesis are not fully revealed. The formation of exosomes seems to be highly conserved and tightly regulated process taking place at endosomal compartments (early endosomes), and matured within multivesicular bodies (MVBs) of late endosomes (Figure 16a). Upon maturation then released to the extracellular environment upon fusion with the plasma membrane and this trafficking is thought to be carried out by Rab GTPases. Collectively, several factors participate in exosome biosynthesis, sorting, maturation and secretion into extracellular milieu (extensively reviewed by Nawaz et al., 2014 [277]).

In addition to exosomes, cells also release other type of vesicles; some are produced through outward budding of plasma membrane (not from endosomes) and direct release from plasma membrane (see [277], and Figure 16b). As compared to exosomes, these vesicles are larger in size and are called microvesicles (MVs). Sometimes apoptotic bodies are also considered as a part of vesicles. Usually cells undergoing apoptosis produces apoptotic blebs and are known as apoptotic vesicles (Figure 16c). Moreover, in recent years there have been also reported even larger membrane derived vesicles, called large oncosomes [346]. Parasites and microbes usually secrete MVs and are commonly known as outer membrane vesicles. International Society for Extracellular Vesicles (ISEV) has designated a generalized term Extracellular Vesicles (EVs) to represent all types of vesicles. 

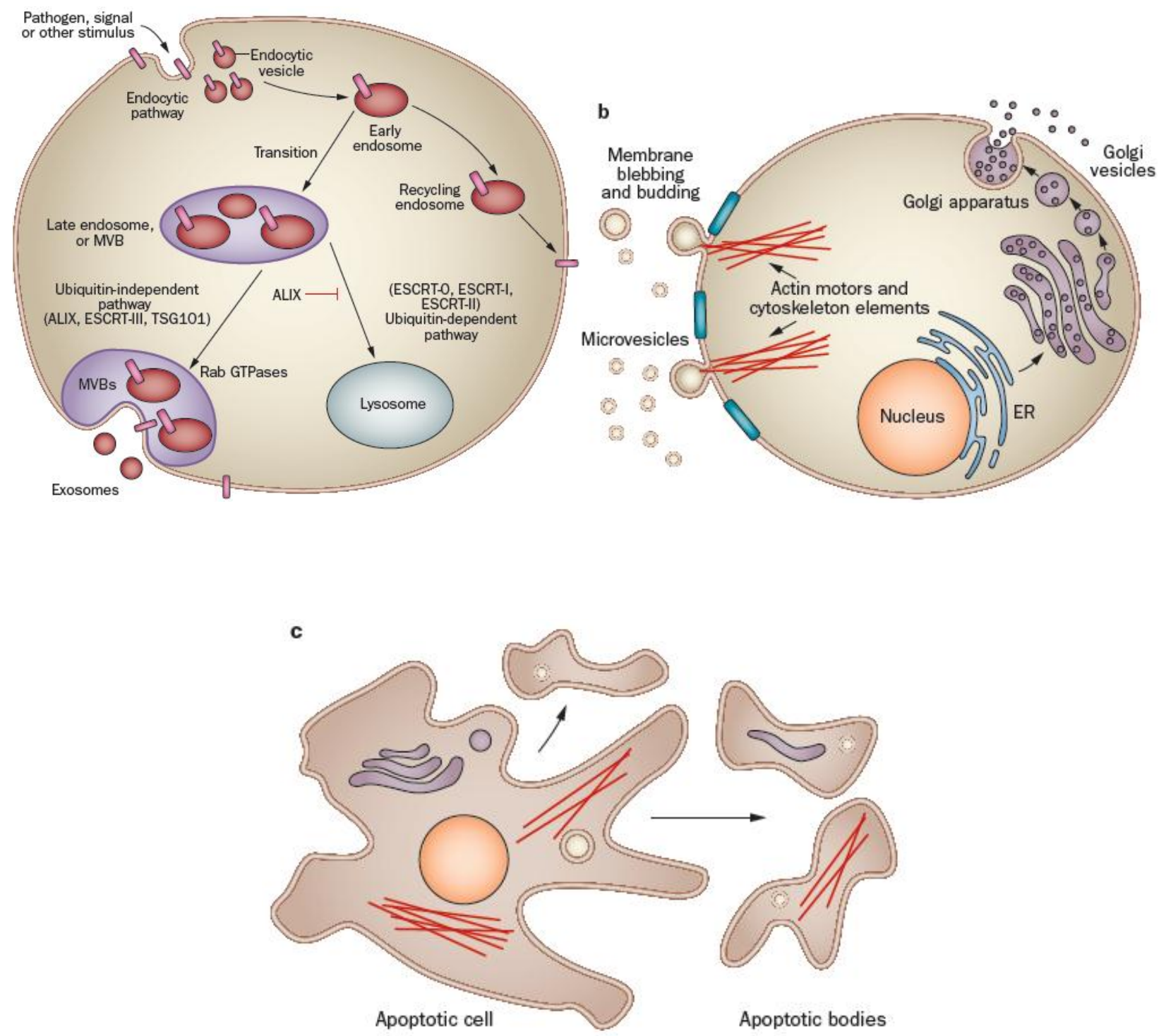

Figure 16: Biogenesis of nanovesicles: (a) Exosomes are formed by endocytosis. These endocytic vesicles mature to early endosomes, and then into late endosomes also known as multivesicular bodies (MVBs). MVBs can be sorted for lysosomal degradation or they can fuse with the plasma membrane and be released outside in the form of exosomes. Rab GTPases regulate MVB fusion with the plasma membrane and release of exosomes. (b) Microvesicles are directly secreted from plasma membrane, whereas (c) apoptotic vesicles are generated through apoptotic protrusion of cells (Adopted from Nawaz et al., 2014, Nature Reviews Urology [277]. 


\subsubsection{Composition of exosomes and molecular contents}

Exosomes are lipid bilayer nanovesicles composed of sphingolipids, cholesterol and ceramide (so called membrane-bound phospholipid nanovesicles). They appear to have round shape or cup shaped morphology when observed under scanning electron microscopy or other fine microscopic technique. Exosomes are best characterized by the surface presence of integrins and tetraspanins such as CD9, CD63, CD81 and cytoplasmic heat shock proteins such as HSP70, and other characteristic proteins including GAPDH, Tsg101 and Alix [347, 348]. These molecules usually serve as exosomal markers to detect exosomes. Exosome surface also contain major histocompatibility complexes such as MHC I and II as well as adhesion molecules. Collectively these molecules define characteristic composition of exosomes.

In addition to characteristic molecules that define exosome composition, exosomes also carry bioactive molecules commonly referred as bioactive contents of exosomes. They carry a repertoire of proteins, including enzymes and variety of transcriptional factors; also the nucleic acids such as DNA including genomic DNA and mitochondrial DNA [349-355], mRNAs [356, 357], miRNAs [357], and other ncRNAs [358], Exosomes also carry several lipids (other than those that compose exosome lipid bilayer), and carbohydrates. The contents of exosomes and other nanovesicles are deposited in vesicle repositories namely Exocarta [348], Vesiclepedia [359], and EVpedia [360, 361].

\subsubsection{Non-coding RNAs in exosomes}

In 2007 Valadi and colleagues for the first time reported that exosomes contain substantial amounts of miRNAs, mRNAs, but little amounts of ribosomal RNAs. More importantly, exosomes are capable of shuttling RNAs between cells; a novel mechanism of genetic exchange between cells [357]. The group also discovered that RNA that is transferred to other cells is functional i.e. mRNA transferred to other cells can be translated into proteins in recipient cells. Translation of mRNA in recipient cells was first time reported by Ratajczak and colleagues [356]. Following this discovery, several other studies later own confirmed the presence of RNA in vesicles and their transfer to neighbouring cells is biologically functional [334, 362, 363]. Pegtel et al., showed that miRNAs secreted by EBV-infected cells are transferred to uninfected recipient cells via exosomes and potentially repress the EBV target genes [327]. Additionally, 
exosomes from dendritic cells (DCs) could fuse with autologous target DCs and efficiently release miRNA into recipient cell cytoplasm where they repress target mRNAs of acceptor DCs [364]. Exosome mediated transfer of ncRNAs is thought a newly evolved mechanism of transregulation between cells, which define enormous potential in physiological as well as pathological states [358]. The detailed roles of ncRNAs in diseases will be discussed in later sections.

\subsection{Isolation, purification and characterization of exosomes}

\subsubsection{Source}

Biological fluids and cultured cell supernatant offer a potential source for isolation of exosomes. Blood plasma or serum is the most commonly used source for exosome collection when it comes to body fluids. According to a recent survey conducted by ISEV, the plasma (47\%), serum $(22 \%)$, urine (14\%), cerebral spinal fluid (8\%) and milk (5\%) are the most common body fluids analysed [365]. The choice of selecting a certain physiological fluid depends on intended downstream analysis [277].

The diverse nature of biological fluids and the individual contaminants of each fluid may represent different molecular combinations outside of exosomes, and may thus require different isolation methods [366]. The diverse nature of biological fluids suggests that exosome cargo of each fluid may represent different composition and therefore, a spectrum of methods needs to be considered to define contaminants of each fluid in order to obtain pure fraction of exosomes.

\subsubsection{Technology for isolation}

There are a variety of methods available and more are being developed, some of them are poorly standardized. These include ultrafiltration, density gradient centrifugation, size exclusion chromatography and affinity isolation, polymeric precipitation and the microfluidic devices [366]. Each method has different isolation efficiencies when applied to different samples, such as blood plasma, milk, urine, and cell culture media.

A comparison of several conventional as well as high throughput technologies for the isolation and characterization of different samples has been recently undertaken with a limelight of their pros and cons [277]. Recently, ISEV has made a critical analysis of various techniques 
implemented for isolation of exosomes and have made very important recommendations [366, 367]. Differential ultracentrifugation remains the most widely used primary isolation method comparable to several other techniques and is suitable for isolation from large-volume of samples. However, for the isolation of exosomes from low volume samples the size exclusion chromatography is now a more widely used technique which allows separation of exosomes from the bulk mix of soluble proteins. In this method the separation is purely based on particle size, therefore contaminating particles in the exosome size range such as lipoprotein complexes may be co-isolated [366, 368]. When intended to capture a selective population of vesicles, the immuno-affinity capture offers and alternative method for higher degree of selection. The method can yield pure vesicle subpopulations (i.e. exosomes, microvesicles), but this method is highly influenced by both the choice of affinity reagent and the ligand density on different populations of vesicles [366].

Other methods include microfluidic devices, filtration and various commercially available kits. The commercial kits could make the use of volume-excluding polymers such as polyethylene glycol (PEG) which enable rapid isolation of vesicles from culture media or body fluids. However, such polymers may also co-precipitate protein complexes that contaminate our isolates. Therefore, the ISEV has recommended different isolation techniques based on different principles each will enrich for different subpopulations of vesicles (separate for exosomes, and microvesicles). Since each method potentially co-isolates the contaminants such as protein complexes and lipoproteins to different degrees, the ISEV proposes the utilization of combinations of techniques, such as density gradient centrifugation followed by size exclusion or immuno-affinity capture [366].

The method of choice should be taken into account based on sample type, volume and the yield, integrity, purity of exosomes required for specific downstream analysis as well as the available instrumentation and processing time [277]. This implies whether the sample is derived from cellculture media or body-fluids, and whether intended for proteomic analysis or nucleic acid profiling. Therefore, the factors of different isolation methods for exosomes have impact on amount, type and purity of exosomes recovered from various sources, as well as may have effect on downstream analysis of vesicles. 


\subsubsection{Technology for characterization}

After the isolation procedures, we need to characterize heterogeneous population of vesicles for their size determination, detection of common markers on the surface of vesicles (as mentioned above in the section of composition of exosomes), morphology and concentration (quantification), for which there is a variety of techniques used [277, 369, 370]. Characterization of exosomes currently present various challenges, mainly due to their small size, complexity of their cargo (contents) and the physical parameters of available instruments for measuring nanosized vesicles.

The size as well as size distribution, and the concentration of individual subpopulations of vesicles (exosomes and microvesicles) is commonly determined by Nanotracking Analysis (NTA), and Zetaview [371-378], and tunable resistive pulse sensing (qNANO) [379]. The electron microscopy is used to assess the submicron phenotype of vesicles [369, 377, 380-382], whereas the flow cytometry is implemented for enumerating, phenotyping and sorting of vesicles based on their size distribution [383, 384]. Western blotting is implemented when aim is to detect exosome markers such as CD63, CD81, and CD9. A recent survey conducted by ISEV has stated that the most widely used techniques for exosomes characterization are western blotting (74\%), single-particle tracking (SPT, 72\%) and electron microscopy (60\%) [365].

Albeit, the flow cytometry remains a popular tool for measuring vesicle populations [383, 384], however fundamental principles and limitations of the instrument needs to be considered [385]. Exosomes isolated by ultracentrifugation may cause aggregation of exosomes thus rendering difficulties in flow cytometric analysis or single particle tracking analysis [365]; whereas, those isolated through kits, might represent difficulty for vesicle analysis with western blotting.

Recently, qNANO based measurements of exosome concentration have demonstrated a standardized method with a feasibility to facilitate comparable and reproducible results that further needs to be validated or reproduced [379].

Additionally, characterizing of heterogeneous subpopulations of vesicles remains an unsolved issue in particular almost all subtypes including exosomes and microvesicles share same/common detection markers such as CD63, CD81 and CD9 [366, 370]. However, to resolve this issue there are recent claims to characterize vesicles based on their surface protein profiling 
at large (proteomics) [375, 386], or RNA content profiling (RNAomics) of individual populations of vesicles separately for exosomes and microvesicles [387-391].

It is expected that new advances in technologies and optimized protocols for the isolation and characterization will certainly foster progress in obtaining pure vesicles. This will greatly influence the identification of specific biomarkers when intended from various diseases as well as therapeutic utility of exosomes.

\subsubsection{Analysis of exosomal nucleic acids}

As stated above that exosomes contain variety of nucleic acids. Prior to profiling of RNA content from exosomes, it is important to assess the quality of RNA. There are predominantly two populations of RNA secreted into extracellular space termed as extracellular RNA (exRNA) (i) either in association with RNA-binding proteins and as a part of lipoprotein complexes (ii) exRNA makes a major fraction into vesicles known as vesicle bound RNA (exosomal-RNA). It has been claimed that protein complexes might be co-precipitated and co-isolated along with exosomes during ultra-centrifugation. Therefore, the methods that remove contaminating protein aggregates from exosome are highly required.

Regarding the presence of exRNA in exosomal forms versus outside exosomes (i.e. non-vesicle exRNA) is a debated subject as there are paradox in the results shown by different labs [392395]. In order to discriminate RNA encapsulated within from those present on the surface of exosomes or non-vesicle RNA, it has been suggested to digest exosome samples with RNase and proteinase to disrupt ribonucleoproteins and RNA exterior to vesicles [366]. This will deplete non-vesicle RNA or the RNA present on the surface of vesicles and will leave behind only the RNA that is encapsulated inside vesicles.

When RNA is isolated from exosomes that were obtained from cell culture supernatant, it may have contaminating RNA species. A potential issue comes from the fact that foetal bovine serum (FBS) that is used for cell cultures; already contains various RNA species. Although, FBS is used after deleting exosomes from FBS, however large fraction is retained even after extended period of ultracentrifugation during the preparations of vesicle-depleted FBS [396]. This raises the possibility that the cell-derived exosomal RNA being analysed might also have RNA from 
FBS exosomes and our qPCR or sequencing analysis might present some artefacts of RNA and may over represent or overestimate our data values [397].

Additional issue that might limit the expression analysis and library preparations from exosomal RNA; is the low input sample material because exosomes have less RNA as compared to cells (in particular from small sample source e.g. less exosomal-RNA from low volume of patient blood or low recovery protocol). Low RNA input material may not only suffer library preparation but also exosomal-RNA ligation biases. Measuring the quantity and integrity of exosomal-RNA is challenging due to low RNA quantities and a lack of standards, such as those established for cell RNA. Detection of the levels of certain transcripts by highly sensitive RTqPCR may be used as a proxy for total RNA quantity in samples containing a very low amount of RNA. Recently, the ISEV has addressed these issues and have made recommendations for the assessment and analysis of the small amount of exosomal-RNAs [366].

Sensitive techniques such as Agilent Bioanalyzer pico chip and the Quant-iT RiboGreen RNA Assay are shown to be more suitable methods for the quantification of exosomal-RNA than that of Nanodrop method. Most of the techniques (with the exception of the Qubit RNA HS Assay), are thought to be sensitive to DNA contamination. Therefore ISEV recommends pre-treatment of samples with DNase for accurate RNA quantitation [366]. Due to the enrichment of small RNA species in exosomes, the main focus of recent studies is the assessment of miRNAs and other small ncRNAs. Such examination takes into account the expression analysis and deep sequencing experiments. ISEV has provided recommendations on the different steps of exosomal-RNA analysis such as RT-qPCR analysis, selection of reference genes, deep sequencing, library preparation, biases issues, data normalization and bioinformatics analysis [366].

\subsection{Biological functions of exosomes}

Although, the functions of exosomes are not illuminated to full extent, however the most profound effect of exosomes that have been invariably established is their participation in cell-tocell communication and signal transduction allowing the exchange of biological information between cells [357, 398-400]. Since eukaryotic cells needs to communicate continuously in order to keep homeostasis, signalling during biological process, nutrients balance and so forth. Now it 
is tempting to escalate that exosomes could mediate bidirectional communication, and therefore the exosome-mediated transport of bioactive molecules could be observed bidirectionally [313]. There is emerging role of exosomes implicated in cellular differentiations, stem cell-maintenance and defining cell-fates (reviewed elsewhere [313]). Such evolving roles of exosomes are mainly reliant on their features that mimic and recapitulate stem cell properties in promoting tissue's intrinsic regenerative programs and repair process within recipient cells in paracrine manner [313].

As compared to other paracrine secreted factors such as cytokines and hormones, the exosomes are gaining intensive attention due to the fact that they transport a variety of bioactive molecules and elicit enormous biological activities between cells (for details see Figure 17 [358]). Exosomes carrying diverse cargo can move through biological fluids in particular through blood circulation and thus may elicit long distance inter organ communication by dissemination of their cargo from one organ to the other one [309, 401]. In addition to regulation of fundamental biological processes and normal physiological states the roles of exosomes have also been increasingly reported in diseases.

\subsubsection{Role in health, normal physiology and disease progression}

Exosomes have attracted much attention in the recent years due to their participation in physiology of the body such as waste management, coagulation, angiogenesis, maintaining homeostasis and host defence [402]. Exosomes mediate physiological messages during reproduction; serve as paracrine factors in maintaining health of paternal and maternal gametes, establishment and continuation of successful pregnancies and health and development of placenta and regulation of maternal immune system [403, 404].

The roles of exosomes are emerging in maintaining cellular health and cell survival including self-renewal, differentiation and pluripotency. Moreover, stem cell derived exosomes are implicated in healing processes by stimulating and regulating intrinsic regenerative programs in damaged tissues. Exosome-mediated functional delivery of RNAs (miRNA, lncRNA and mRNA) to the sites of injury could enable regulation of genes responsible for repair processes in injured cells [313]. Exosomes derived from stem cells could potentially exert immunomodulatory and anti-inflammatory effects, anti-apoptotic and protective effects in order 
to ameliorate organ functions [312]. Interestingly, exosomes play active roles as messengers during development of central nervous system as well as pathophysiology of the brain both in healthy and diseased brain [405-411].

Although exosome secretion is a constitutive process, however the process of release may be accelerated during altered cellular or organ conditions and disease state, such as against infectious agents, or during inflammatory and immune responses. Due to their natural capacity in transportation and dissemination of biological molecules exosomes could spread toxic and misfolded or abnormally expressed proteins, lipids, mutated genes and deregulated nucleic acids. Therefore, they can propagate number of diseases such as neurodegenerative disease [412-414], inflammatory and cardiovascular diseases $[415,416]$. In addition, exosomes could mediate hostparasite interactions and may contribute in the progression of infectious diseases by disseminating virulence factors [417]. Additionally, exosomes may have profound effect on immunomodulation including both immune suppression as well as immune activation [303, 312, $418]$.

\section{Exosomes as mediators of cancer progression}

Since, exosomes carry several regulatory transcriptional factors, proteins and regulatory RNAs and transferred from donor cell to recipient cells-their role have been implicated in oncogenesis [419]. Exosomes released from cancer cells may have mutated genes or oncogenes therefore; exosomes can contribute to a horizontal propagation of oncogenes and their associated transforming phenotype among subsets of cancer cells. This is important to consider that metastatic potential of exosomes is greatly reliant of dissemination of abnormally expressed bioactive molecules such as proteins, genomic and mitochondrial DNA, transposon elements, and mutated genes [334, 349, 354, 363, 419-424].

Exosomes could travel to distant sites in the body or could become part of circulation and may fuse with specific cells at these distant sites. These interactions could modulate distant microenvironment making it suitable for the development of new tumour cells. Thus exosomes can 'educate' cells or organs at distant to become receptive for metastases [421]. Exosomes are recently being reported as facilitators of cancer cells for their co-evolution with tumour microenvironment and metabolic co-option [425, 426]. Other mechanisms of exosome-mediated 
tumour progression may include stromal remodelling, immune evasion, neovascularization and metastasis [312, 401, 427-432].

\subsubsection{Exosome associated ncRNAs and cancer development}

Arguably, the exosome-mediated transport of ncRNAs is thought to present more diverse regulatory functions as compared to other contents of exosomes. This is because of the fact that ncRNAs regulate gene expression at large and are capable of modulating genetic profiles of recipient cells and may foster genomic instability [358]. As such, the transport of ncRNAs between cells may consequently result into diverse cascade of events leading to progression of cancer (Figure 17). The dissemination of cancer cell-derived ncRNAs via exosomes could reinforce cancer cells to exhibit resistance to therapies. In short, exosomes carry distinct patterns of ncRNAs secreted from cancer cells and exhibit distinct regulatory features in the context of cancer progression. Such roles of exosome-linked ncRNAs have been extensively reviewed [358]. 


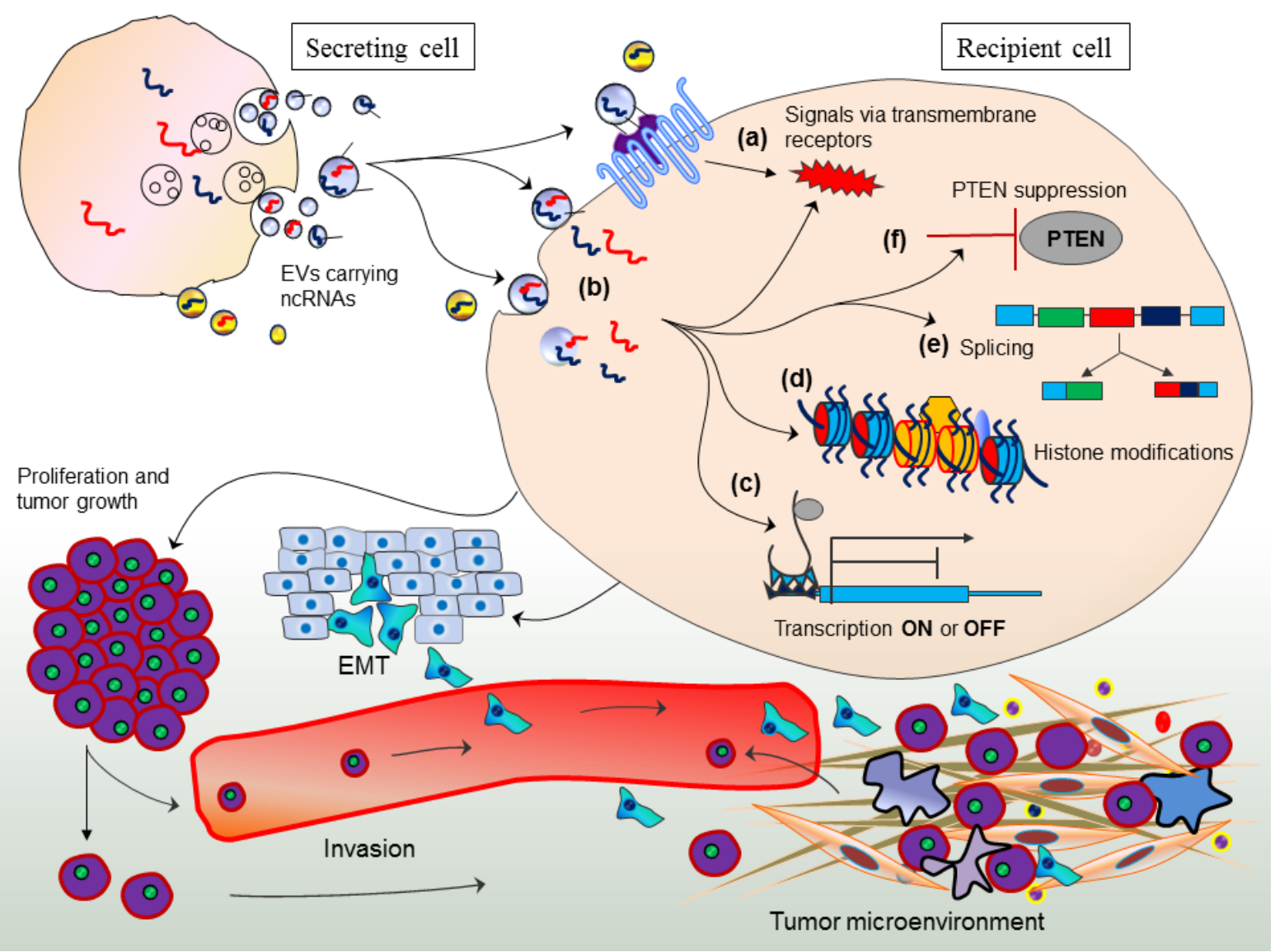

Figure 17. Mechanisms of exosome mediated transport of non-coding RNAs and tumour progression. Exosomes deliver non-coding RNAs from cancer cells to other cells and elicit wide range of genetic programs in recipient cells, which lead to progression tumour. Figure adopted from Fatima, F. and Nawaz M., 2017 [358]. 


\subsection{Exosomes as diagnostic and prognostic biomarkers}

Proteins and nucleic acids encapsulated within exosomes are thought to be highly stable against degradation by proteases and nucleases that are naturally present in body fluids. Such protection provides a great advantage for storage conditions and handling at adversative physical conditions such as multiple freeze and thaw cycles, fluctuations in temperature and changes in $\mathrm{pH}$, making exosomes as appealing source for biomarker development [277, 311]. Moreover, circulating exosomes in body fluids of cancer patients having tumour specific signatures could provide a platform of biomarker discovery. Importantly, the contents and profiles of cancer derived exosomes are significantly distinct as compared to normal controls [433-435].

Exosomes released into body fluids not only contain differentially or aberrantly expressed miRNAs but also often retain the characteristics of the tumours from which they originated [334, $434,436]$. It has been proposed that the cellular origin of exosomes from certain cancer types or cell types could be used to determine the likely identity of an unknown disease [437]. Consequently, exosomes obtained from body fluids could be used to large-scale screening of tumour specific markers [311, 349, 438-442]. Interestingly, exosomes could be easily isolated from various body fluids such as blood plasma, serum and urine. Samples could be collected longitudinally with great ease and in large quantities (i.e. sample reproducibility), providing a tool for liquid biopsies. Exosome release is an active process and tumour cells can shed plenty of exosomes per mL of plasma [439].

In this context, the presence of ncRNA in exosomes, they may serve an additional platform for biomarker discovery [358, 443]. The global profiling of exosome-encapsulated RNAs with mutation characterization and/or without mutation characterization could serve potential source for cancer detection. This could allow clinicians to perform patient stratification (companion diagnostics), screening, monitoring treatment response and detection of minimal residual disease after surgery/recurrence. In addition to their utility in diagnostic platforms, the exosomeassociated ncRNAs from peripheral blood could serve as bona fide markers of disease prognosis, tumour recurrence and overall survival. This refers to prognostic implications against chemo therapies as well as radio therapies related to several cancer types [358]. Therefore, the ncRNA signatures from exosome fractions may have clinical implications. 


\subsection{The therapeutic applications of exosomes}

In fact, some of the earliest exosome research indicated secretion of exosomes could promote antitumor immune responses in mice in vivo [303]. When applying exosomes as therapeutic vectors they are advantageous in the context that exosomes do not invoke an immune response in host. Alternatively, they regulate immune responses in such that they could be exploited for immunotherapies [311, 418]. Exosome therapies are now being explored in anti-cancer clinical trials as recent reports claim taxol-filled exosomes can be used to treat cancers in mice with 50fold lower doses than conventional treatments [444]. Exosomes are also being implicated in repairing the defects and stem cells therapies $[312,445]$, with enormous potential in clinical settings $[446,447]$.

Exosomes have been proposed to be superior delivery vectors as compared to synthetic polymers, due the fact that exosomes are naturally occurring biological entities, and are well tolerated by the host as compared to other delivery vectors. They do not evoke immune responses in host, easy to prepare, cost effective and have no potential ethical issues, therefore have emerged as alternative platform in nano-medicine. Inspired from their natural ability in transporting genetic material between cells, exosomes can be used as vehicles of gene delivery. However, to apply exosomes as delivery vectors, first of all drug or therapeutic RNA needs to be loaded into exosomes and the loading efficacy must be defined prior to their internalization into target cells.

Researchers have loaded therapeutic interfering RNAs (siRNA) into exosomes and have administered them as gene delivery vectors in vitro as well as in vivo $[448,449]$. However, the specific targeting and internalization of exosomes by intended organs remains a critical question. More refined methods for exosome tailoring, as well as routes of administration are prerequisite. In addition, defining the doses to each separate disease type and whether the dose-dependent effects of drugs or therapeutic RNA administered through exosomes in model animal experiments is promising must be determined. However, this is truly interesting to use exosomes as safe delivery vectors. 
AIMS AND OBJECTIVES 


\section{AIMS AND OBJECTIVES}

Exosome-vesicles are important messengers of cell-to-cell communication and potentially exchange genetic messages between cells by transporting nucleic acids in particular ncRNAs. Albeit much is known about the presence of RNA molecules in exosomes, little is known about the mechanisms by which these RNAs are packaged into exosomes. The major aim of this study (performed at University of Gothenburg Sweden as a part of $\mathrm{PhD}$ sandwich program) was to study the mechanisms by which RNA molecules are packaged into exosomes. A research article has been submitted to a peer reviewed scientific journal (but data is not shown in thesis).

Since exosomes vesicles naturally shuttle/deliver RNAs between cells (first time revealed by Valadi and colleagues at University of Gothenburg Sweden [357]; the aim was also to use these vesicles as gene delivery vectors. Data is not shown in thesis (as the Swedish group has patent application).

As the content of exosomes varies depending on cell types, cellular conditions or disease state such as cancer; we aimed also to examine cancer specific contents including RNA and proteins for identification of cancer biomarkers.

The more specific objectives are;

1. Isolation of exosome-vesicles from cancer cells and analysing RNA content in exosomes

2. Isolation of exosome-vesicles from cancer cells and analysing protein content in exosomes

3. Studying the mechanisms of RNA packaging into exosomes

4. Using exosomes as gene delivery vectors

5. Determining the potential biomarkers from cancer cell derived exosomes 
METHODOLOGY 


\section{MATERIALS AND METHODS}

\subsection{Preparations of exosomes from supernatant of cancer cell lines}

\section{Cells}

Lung cancer cells line (HTB-177) and breast cancer cell line (HTB-133) were purchased from ATCC (USA) and cultured as described by ATCC guidelines with some modifications. The growth medium RPMI-1640 medium (Sigma Aldrich, Sweden) was supplemented with 10\% foetal bovine serum (FBS), $2 \mathrm{mM}$ L-glutamine and 1\% penicillin/streptomycin (ThermoFisher Scientific, Sweden). Prior to its supplementation with medium, the FBS was depleted from exosomes by ultracentrifugation using 70Ti rotor (Optima L-100 XP Beckman Coulter ultracentrifuge). The cultured cells were harvested after reaching $~ 90 \%$ confluency for the isolation of exosomes.

\subsection{Isolation and purification of exosomes}

Exosomes were isolated from HTB-177 cell lines through a series of ultracentrifugation steps accompanied by microfiltrations with some modifications from what has been previously described previously [448]. Briefly, the conditioned culture medium was harvested and centrifuged (SIGMA 4K15) at $1300 \mathrm{rpm}$ for $15 \mathrm{~min}, 4^{\circ} \mathrm{C}$ in order to eliminate the cell debris followed by ultracentrifugation at $19800 \mathrm{rpm}$ for $30 \mathrm{~min}$ and $4^{\circ} \mathrm{C}$. The resultant supernatant was filtrated through a $0.22 \mu \mathrm{m}$ filter and was ultracentrifuged at $38800 \mathrm{rpm}$ for $70 \mathrm{~min}$, allowing the final isolation of the exosome pellet. All the ultracentrifugation steps were performed by using a Beckman 70Ti rotor (Optima L-100XP Beckman Coulter ultracentrifuge). The isolated exosomes were dissolved in PBS for protein estimation and were directly used for RNA isolation.

\subsection{Exosome estimation}

Exosome pellet(s) were dissolved and resuspended in $10 \mu \mathrm{l}$ PBS and $10 \mu \mathrm{l}$ SDS was added to it. $1 \mu 1$ exosome sample solution was dissolved in $198 \mu$ l of Qubit buffer and $1 \mu 1$ protein reagent. The readings were made using Qubit 2.0 spectrofluorometer (Thermo Scientific). 


\subsection{RNA isolation from exosomes}

The total RNA from exosomes as well as from parent cells was isolated using miRCURY ${ }^{\mathrm{TM}}$ RNA isolation kit (Exiqon, DK) according to the manufacturer's instructions and as described previously [450]. RNA quantification and quality control were performed by using both NanoDrop v2.0 (Thermo Scientific) and Qubit 2.0 spectrofluorometer (Thermo Scientific).

\subsection{Detection of exosomes by exosomal markers}

The isolated Exosomes were detected by exosomal markers using both western blotting and FACS scan against CD63, CD9, and CD81 as described previously [448].

The results showed that the exosomes carry all the three markers commonly used for exosomes detection.

\subsection{Identification of proteins}

Next, in order to investigate whether the protein content of these two types of exosomes, derived from breast and lung cancer cells, can potentially be used as biomarker, we have identified the total protein content of these exosomes using magnetic beads coupled with antibodies against the MHCII proteins. Exosomes isolated this way, using antibody-coupled-magnetic beads, turned to be more pure from the sticky proteins such as albumin.

For the identification of proteins in exosomes, the liquid chromatography-tandem mass spectrometry (LC-MS/MS) was used as described previously [357, 451, 452], with little modifications.

\subsection{Network analysis of proteins identified in HTB-133 and HTB-177 exosomes}

Network analysis of the identified proteins present in exosomes derived both from breast cancer cells HTB-133 and lung cancer cells HTB-177 was performed. Ariadne pathways analysis software (http://www.ariadnegenomics.com/products) was used to analyse the identified exosomal proteins. 


\section{RESULTS}




\section{RESULTS}

\subsection{Detection of exosomal markers}

The western blotting and FACS scan against CD63, CD9, and CD81 showed that the exosomes carry all the three markers commonly used for exosomes detection (Figure 18).

(a)
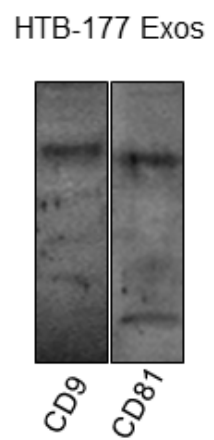

(b)

HTB-133 Exos

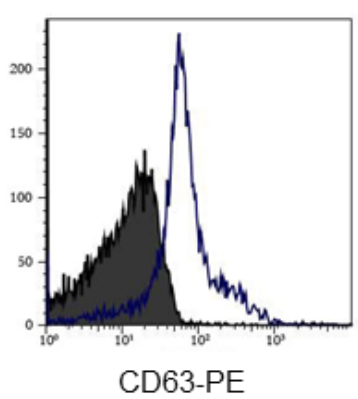

Figure 18: Detection of exosomal markers: (a) Exosomes from HTB-177 lung cancer showing CD9 and CD81 markers. (b) Exosomes from HTB-133 breast cancer showing the detection of CD63 marker. 


\subsection{Detection of RNA in exosomes versus cells}

The exosomes were derived from two different cancer cells; lung cancer cells HTB-177 and breast cancer cells HTB-133. Cell RNA and exosomal-RNA was analysed by Bioanalyzer and comparison was made between cellular and exosomal-RNA. Figure 19a and 19b shows cellular RNA from HTB-177 lung cancer cells and HTB-133 breast cancer cells respectively. Figure 19c and 19d shows exosomal-RNA derived from HTB-177 lung cancer cells and HTB-133 breast cancer cells respectively.

(a)

Cell RNA HTB-177

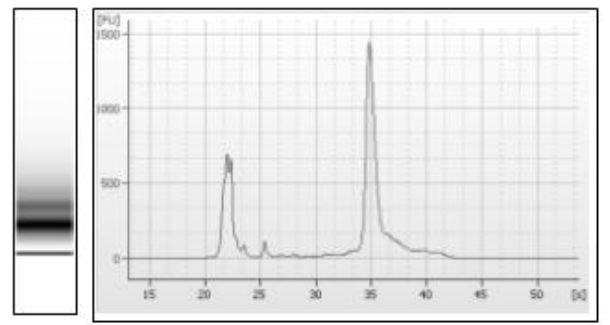

(c)

Exosomal-RNA from HTB-177 cells

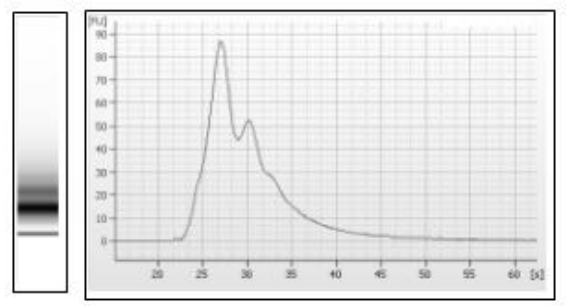

(b)

Cells RNA HTB-133

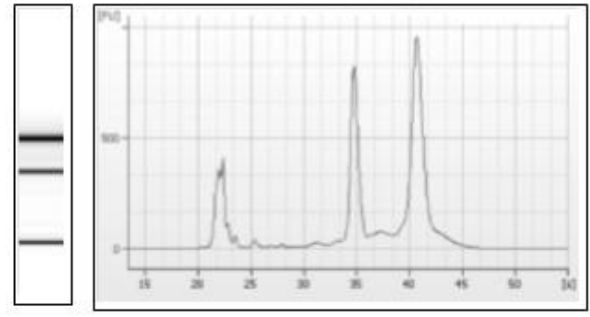

(b)

Exosomal RNA from HTB-133 cells

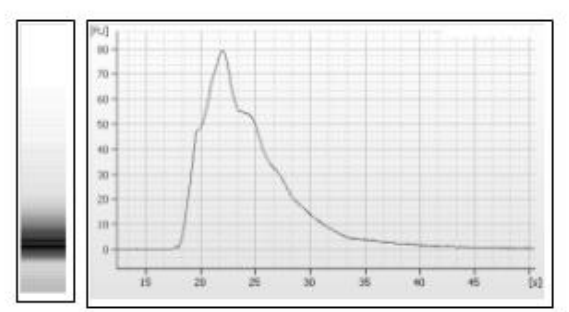

Figure 19: The comparison of RNA in cells and exosomes. (a \& b) Cellular RNA from HTB177 lung cancer cells and HTB-133 breast cancer cells respectively. (c \& d) Exosomal RNA derived from HTB-177 lung cancer cells and HTB-133 breast cancer cells respectively. 


\subsection{Comparison of total RNA and proteins from exosomes versus cells}

The exosomes isolated after 4 days (90\%) cell confluency, were estimated for total RNA isolated from exosomes and were compared with cellular RNA. Results show that there is differential presence of RNA in exosomes versus cells. Similarly cellular proteins and exosomal proteins showed differences (Figure 20). Additional analysis for specific miRNAs and mRNA presence in exosomes and expression was evaluated by RT-qPCR and compared with cellular expression levels of miRNA. The results have shown that there is a preferential sequestering of some miRNAs into exosomes having their levels different from parent cells.

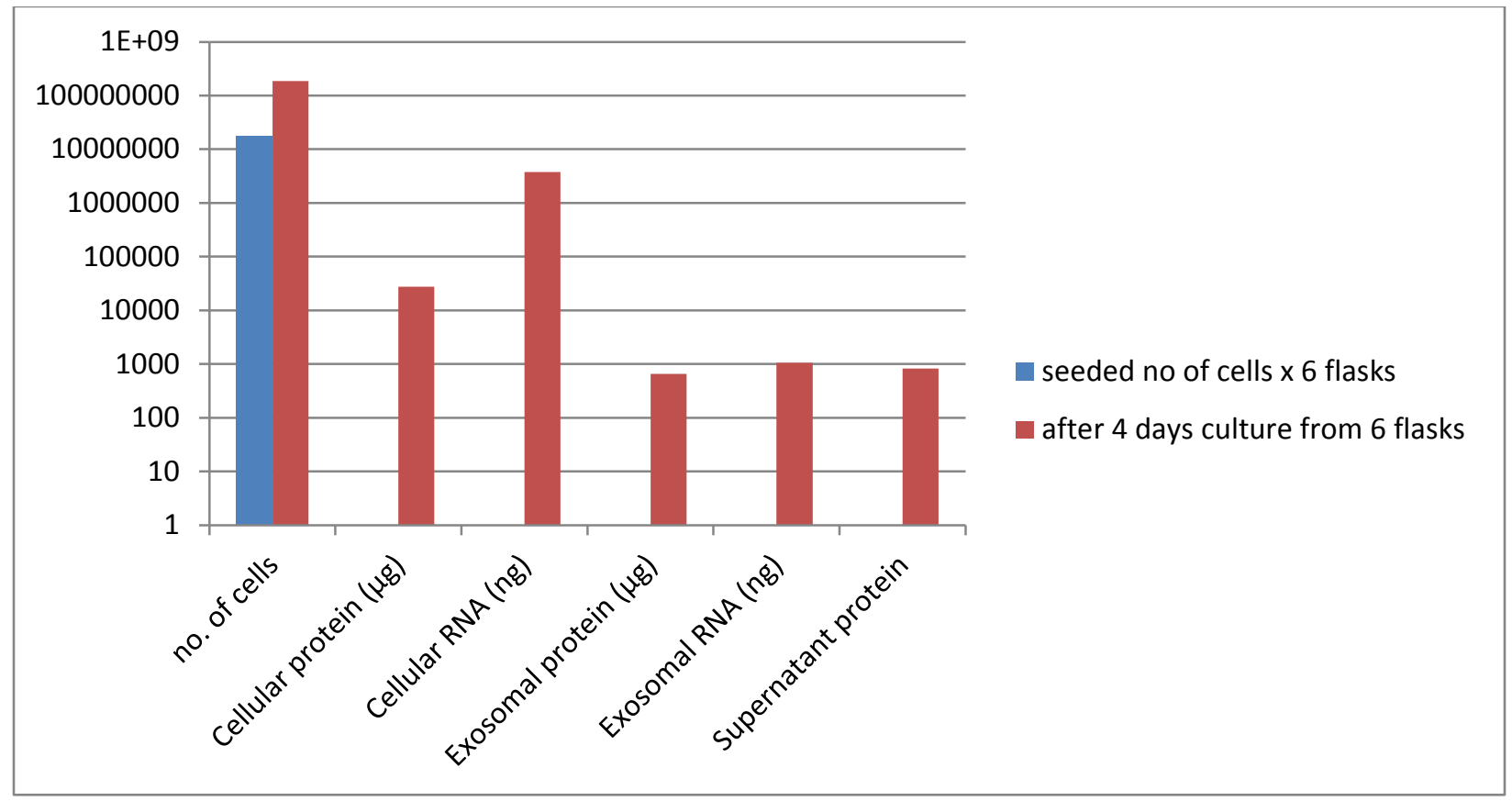

Figure 20: Estimation and comparison of exosomal RNA and exosomal proteins with cellular RNA and protein 


\subsection{Identification of proteins}

Next, in order to investigate whether the protein content of these two types of exosomes, derived from breast cancer (HB-133) and lung cancer (HTB-177) cells, can potentially be used as biomarker, we have identified the total protein content of these exosomes using magnetic beads coupled with antibodies against the MHCII proteins (Figure 21). Exosomes isolated this way, using antibody-coupled-magnetic beads, turned to be more pure from the sticky proteins such as albumin.

Using liquid chromatography-tandem mass spectrometry (LC-MS/MS), in total 192 proteins were identified in exosomes derived from the breast cancer cells. $74.5 \%$ of them have at least 2 hits, and at least one hit with $99 \%$ accuracy, whereas, $25.5 \%$ of them have at least one hit at $99 \%$ which is equivalent to 49 proteins out of 192 proteins totally identified.

In total, 166 proteins were identified in exosomes derived from the lung cancer cells. $68 \%$ of them have at least 2 hits, and at least one hit with 99\% accuracy. 32\% of them have at least one hit at $99 \%$ accuracy which is equivalent to 35 proteins out of 166 proteins identified totally. Out of total (192 proteins in breast cancer exosomes and 166 lung cancer exosomes), 75 proteins were found common in both exosomes. Comparison is made in Figure 22. 


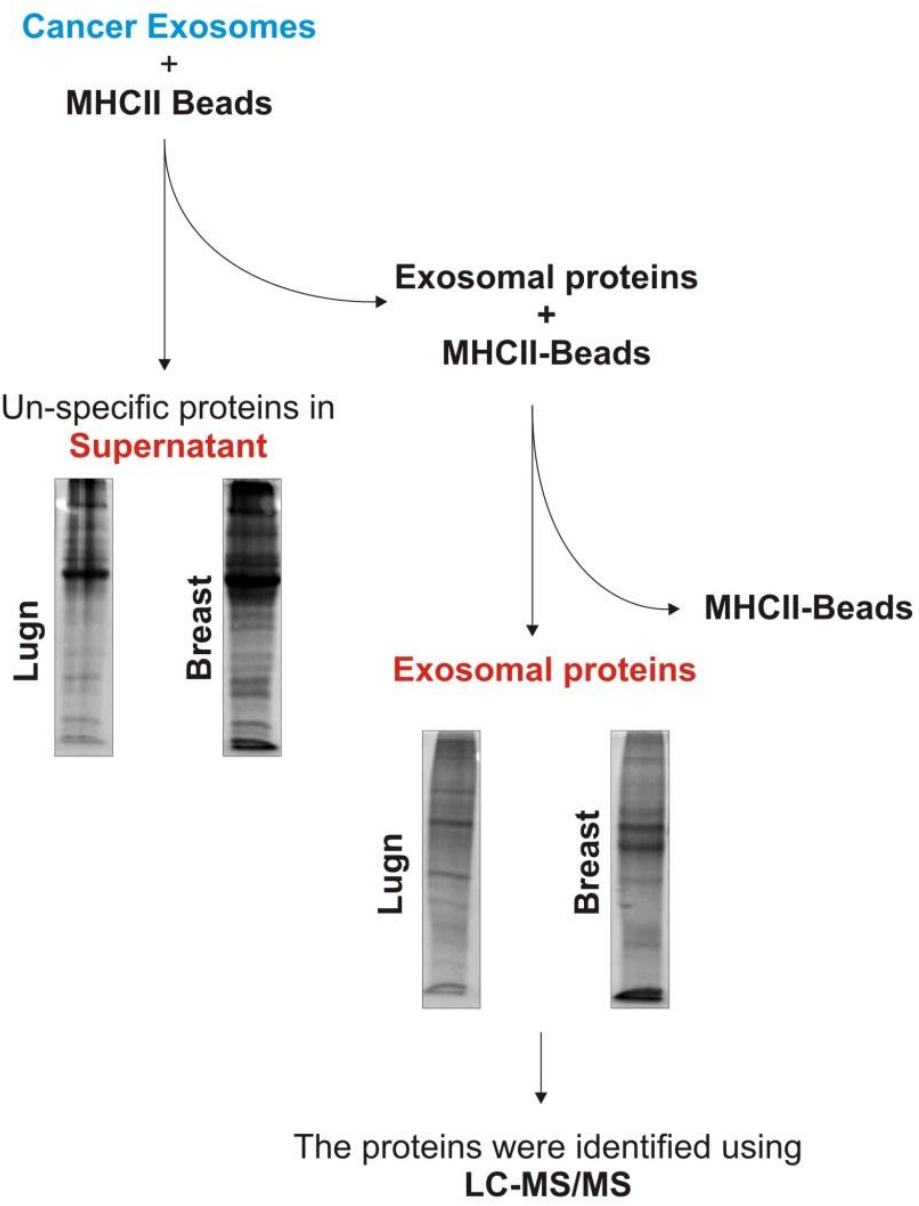

Figure 21: Scheme for identification of the total protein content from exosomes 


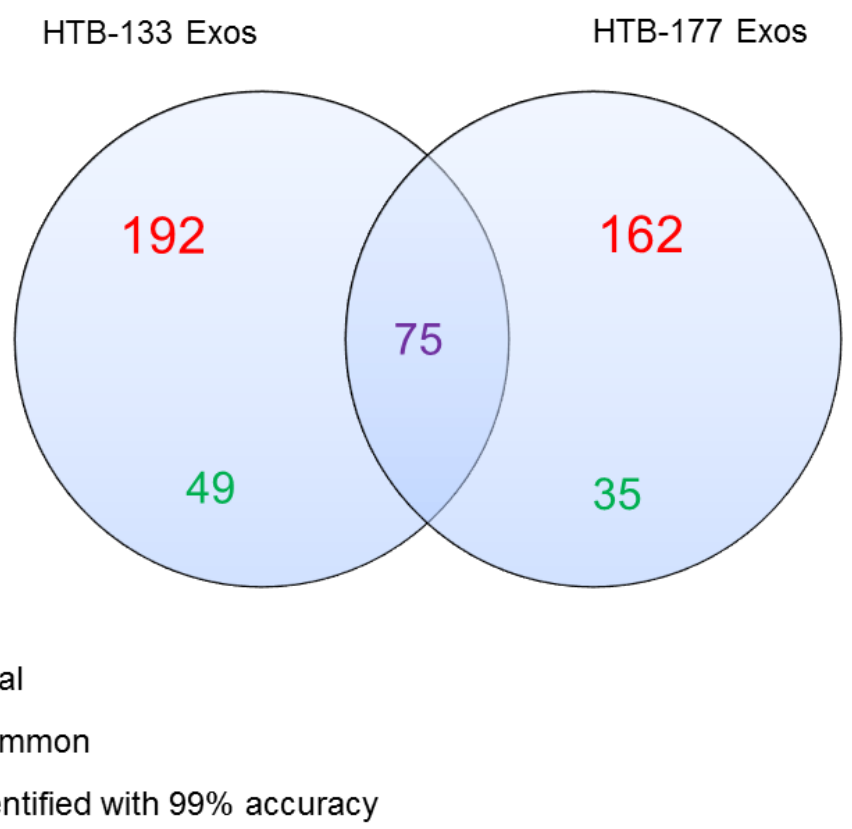

Figure 22. Proteins identified in exosomes from HTB-133 breast cancer cells and HTB-177 lung cancer cells. In total 199 proteins were identified in exosomes from HTB-133, and total 166 in HTB-177. 75 proteins were found common in exosomes from both sources. Of 192 total proteins in HTB-133 exosomes, 49 proteins showed at least 1 hit with 99\% accuracy. Likewise, of total 162 proteins in HTB-177 exosomes, 35 proteins showed at least 1 hit with 99\% accuracy. 


\subsection{Network analysis of proteins identified in HTB-133 derived exosomes}

Network analysis of the identified proteins present in exosomes derived from breast cancer cells HTB-133. Ariadne pathways analysis software (http://www.ariadnegenomics.com/products) was used to analyse the identified exosomal proteins (Figure 23).
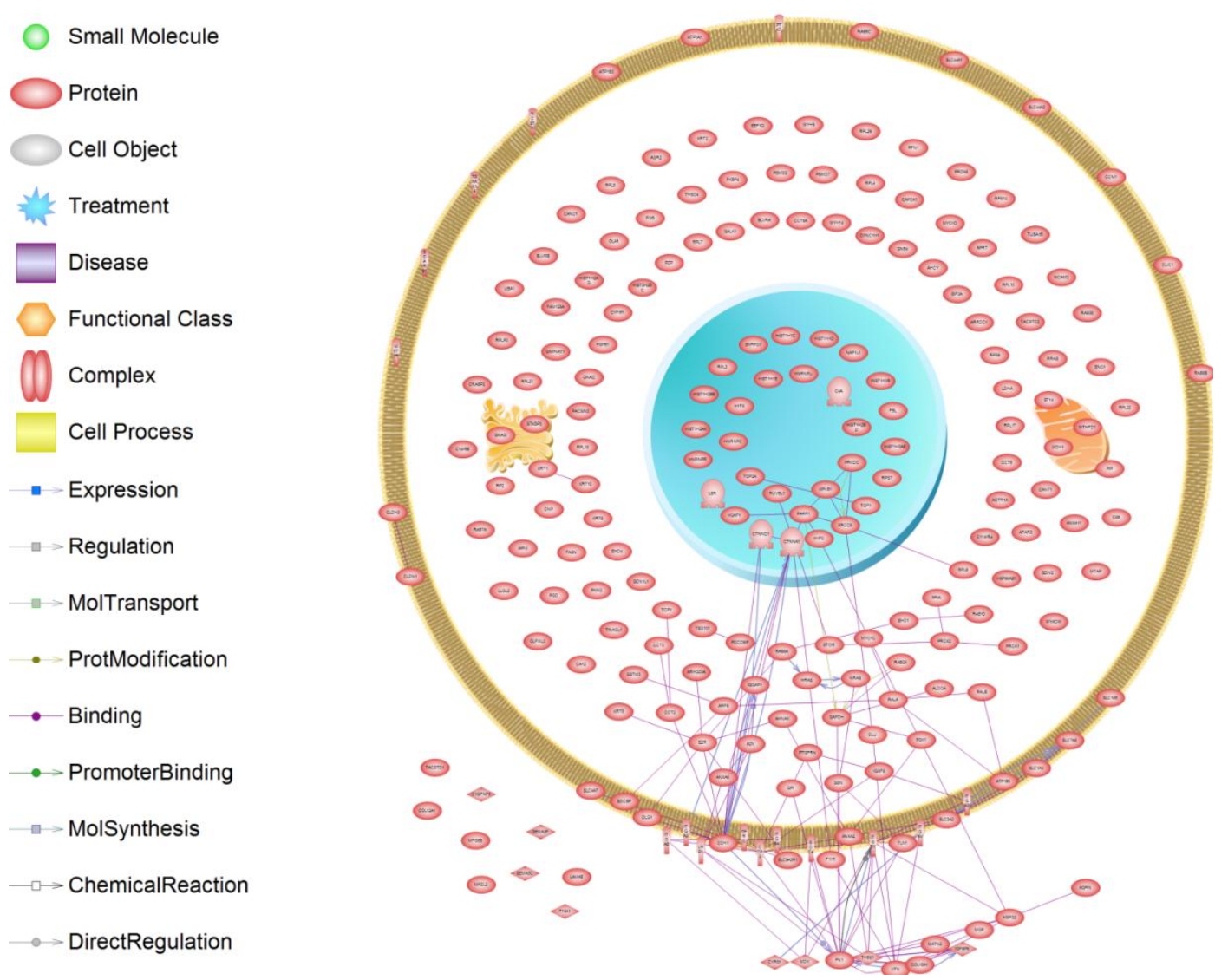

Figure 23. Network analysis of the identified proteins present in exosomes breast cancer cells HTB-133. 


\subsection{Network analysis of proteins identified in HTB-177 derived exosomes}

Network analysis of the identified proteins present in exosomes derived from lung cancer cells HTB-177. Ariadne pathways analysis software (http://www.ariadnegenomics.com/products) was used to analyse the identified exosomal proteins (Figure 24).
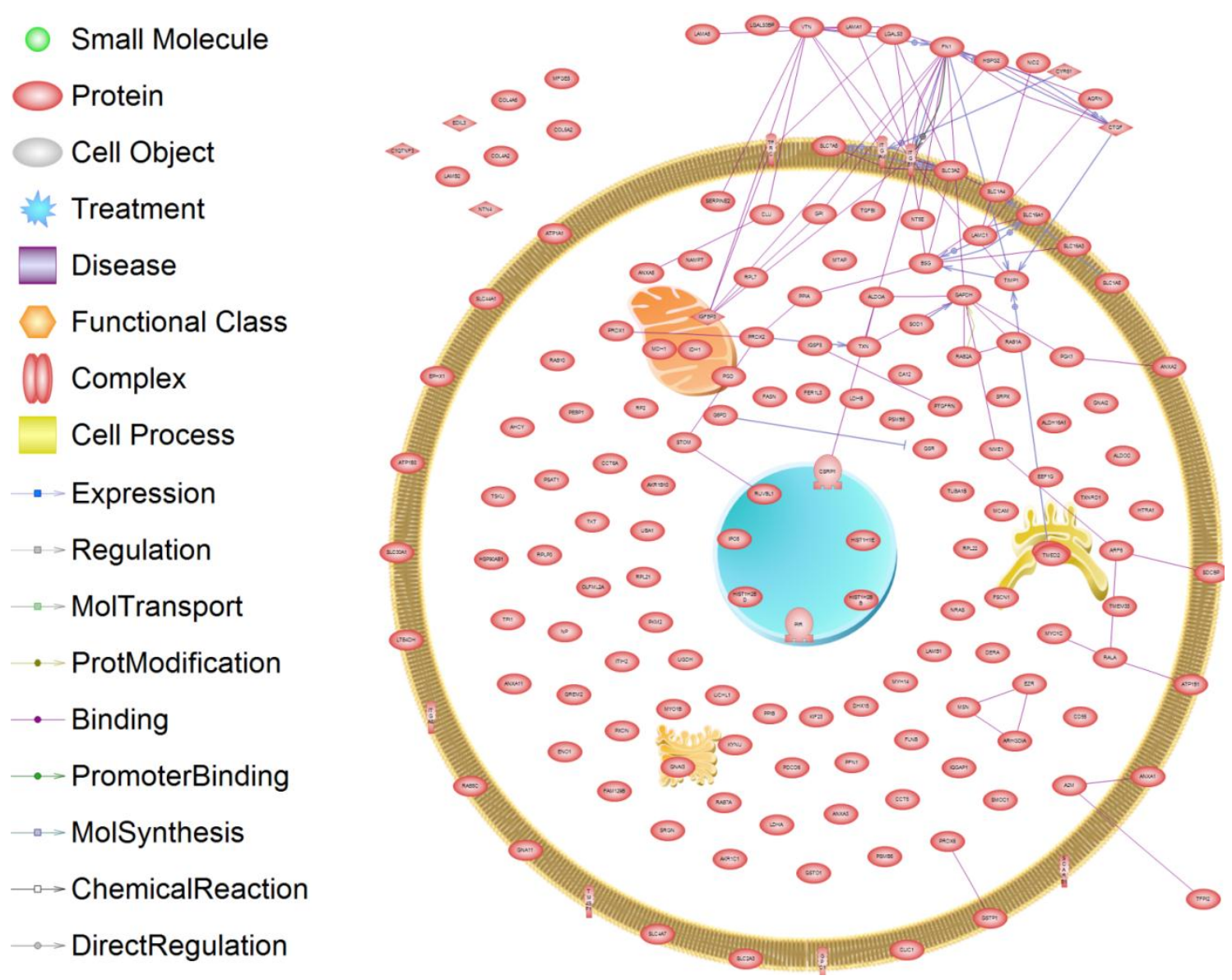

Figure 24. Network analysis of the identified proteins present in exosomes derived from lung cancer cells HTB-177 


\section{DISCUSSIONS}




\section{DISCUSSIONS AND CONCLUDING REMARKS}

Exosomes vesicles are important messengers of cell-to-cell communication and potential vehicles of genetic exchange between cells by transporting nucleic acids. Due to their wide spread role in dissemination of bioactive molecules, exosomes are implicated in variety of diseases. Albeit much is known about the presence of RNA molecules in exosomes and their transport to other cells, little is known about the mechanisms by which these RNAs are packaged into exosomes (recall that exosomes are synthesised by endosomal pathway). There are initial studies which propose that both active and passive mechanisms involved in the incorporation of RNA molecules into endosomes [453-455], however the detailed mechanisms are not fully known. In our study (part of PhD sandwich program performed at University of Gothenburg) we revealed new mechanisms of RNA packaging into exosomes and identified the role of several RNA binding proteins (Article submitted to a peer reviewed scientific journal, but data not shown as the results are protected by host group at University of Gothenburg).

Perhaps the most interesting feature for which exosomes have received intensive attention is their natural ability to act as vehicles of genetic transport. Since exosomes naturally shuttle/deliver RNAs between cells (first time revealed by Valadi and colleagues from University of Gothenburg Sweden [357]); the exosomes can be used a vehicles of gene delivery to target cells as toots for gene therapy. For gene therapy several vectors have been developed in last decades such as viral vectors and liposomal vectors, gold or carbon nanoparticles, and synthetic polymers. However, exosomes are thought to be superior that existing methods of gene therapy, because exosomes are biological entities secreted from cells and these vesicles are naturally engineered biological vectors which do not evoke immune responses in the host. Exosomes are easy to obtain, easy to handle and are cost effective.

Major limitation is loading RNA of interest into exosomes. Since this field is new and there are very few reports that have tried to develop methods for loading therapeutic RNAs into exosomes. Researchers have loaded therapeutic interfering RNAs (siRNA) into exosomes and making attempts to using exosomes as gene delivery vectors in vitro as well as in vivo [448, 449]. However, these methods are insufficient in terms of loading efficacy and delivery efficiency. Therefore, more improved methods are required. We (a part of PhD sandwich with Hadi Valadi, at University of Gothenburg) developed a more advanced method of loading therapeutic RNAs 
into exosomes as well as their delivery to cancer cells in-vitro and in-vivo. As compared to previous methods this method is more robust, efficient and uses advanced technology (Data is not shown, as the results are protected by group).

As the content of exosomes varies depending on cell types, cellular conditions or disease state such as cancer [277, 311]; therefore we aimed to examine cancer specific contents including RNA and proteins for identification cancer biomarkers and their relevance to diseases progression. Exosomes isolated from different cancer cells were shown to be positive for exosome detection markers CD9, CD63 and CD81 confirming that our preparations have exosomes. The analysed of exosomes from breast cancer cells and lung cancer cells showed that different cancer types secrete different amount of RNA and proteins. Moreover, not only the amount but also the type of contents is different in different cancer cell types.

Interestingly, the RNA in exosomes shows selective sequestering/packaging, as it was observed by setting comparison between cellular RNA and exosomal-RNA. The absence or little presence of ribosomal RNA in exosomes indicates that cells mostly secrete miRNAs or mRNAs but not the ribosomal RNAs. This implies that cells have evolved a mechanism of packaging of functional RNA (mRNAs, mRNAs but not the ribosomal RNAs) into exosomes and delivery to other cells. Also, such trends are unique to exosomes from individual cancer type, as was shown by comparing breast cancer-secreted exosomes and lung cancer secreted exosomes. It can be inferred that the secretion of cancer specific miRNAs in exosomes could be used as disease biomarkers, whereas their delivery to other cells may help understand the mechanisms of gene regulation between cells.

Since long it has been thought that cells releases secretory proteins into extracellular environment. However, it is only recently being explored that cells also secrete proteins via exosomes. In current study the proteins identified from two different exosomes sources (i.e. rom breast and lung cancer cells) were different in both types of exosomes and some were highly specific to each exosome type. However, the identification of proteins that are common in exosomes from breast cancer and lung cancer indicate that they might have some common roles in cellular functions. The networking and pathway analysis of proteins identified from two types of exosomes, indicate their participation in various signalling pathways linked to cell cycle 
regulation, adhesion, migration and proliferation, as well as matrix remodelling in order to prepare tumour microenvironment. This suggests that cancer cells secrete proteins to communicate tumour related messages to other cells. However, to understand their mechanisms will need further studies in certain in-vivo disease models and targeting specific pathways.

Although, exosomes have enormous potential for their utility as diagnostic and prognostic biomarkers, however there are certain technical challenges that need explicit attention. Pertinent to using exosomes as diagnostic biomarkers, the harvesting pure exosomes from patient body fluids still needs more sensitive capture platforms, standardized and optimized methods. As discussed in previous sections that exosomes are contaminated with lipoproteins or other protein complexes. Also, exosomes may have sticky proteins such as albumin. In this regard, the identified protein content from exosomes using magnetic beads coupled with antibodies against the MHCII proteins, turned to be more pure from the sticky proteins such as albumin. However, protein contamination is not the only issue; there are other potential issues for exosome isolation, purification and characterization (as discussed in introduction section).

The applications of highly sensitive detection platforms and high-throughput next generation tool may warrant the utility of exosomes as routine biomarkers. There have been suggested the strategies to rigorously validate and test exosomes for applying US food and drug administration (FDA) approval [277]. The emerging technology for obtaining clinical grade and pure exosomes will help to use exosomes as diagnostic biomarkers and their applications as nano-therapeutics [358, 418]. Since this field is gaining huge interest both in basic research in and translational medicine, there are several methods and technology is being developed by newly formed companies specific for exosome research.

Finally, the therapeutic applications of exosomes as drug delivery or gene delivery vectors are promising. By having improved methods of drug loading into exosomes and defining their therapeutic routes of administration in disease models will make exosomes better defined therapeutic modalities. In addition, defining the doses to each separate disease type and whether the dose-dependent effects of drugs or therapeutic RNA administered through exosomes in model animal experiments is promising must be determined. The evaluation of exosomes for clinical trials in human diseases has been already started and exosomes have approved for phase II, and -III clinical trials [446, 447, 456]. This takes exosomes applications from bench to 
bedside. There is intensive interest in the field of exosomes and recent decade has witnessed huge amount of scientific reports on exosomes both in understanding basic functions of exosomes as well as their therapeutic applications. It is anticipated that the next decade will form the basis of advanced and targeted therapeutic strategies and this is truly interesting area of research. 
REFERENCES 


\section{REFERENCES}

1. Buckner, J.C., et al., Central nervous system tumors. Mayo Clin Proc, 2007. 82(10): p. 1271-86.

2. Louis, D.N., et al., The 2007 WHO classification of tumours of the central nervous system. Acta Neuropathol, 2007. 114(2): p. 97-109.

3. Louis, D.N., et al., The 2016 World Health Organization Classification of Tumors of the Central Nervous System: a summary. Acta Neuropathol, 2016. 131(6): p. 803-20.

4. $\quad$ Siegel, R.L., K.D. Miller, and A. Jemal, Cancer statistics, 2016. CA Cancer J Clin, 2016. 66(1): p. 730.

5. Nikiforova, M.N. and R.L. Hamilton, Molecular diagnostics of gliomas. Arch Pathol Lab Med, 2011. 135(5): p. 558-68.

6. Gilbertson, R.J. and J.N. Rich, Making a tumour's bed: glioblastoma stem cells and the vascular niche. Nat Rev Cancer, 2007. 7(10): p. 733-6.

7. Cancer Genome Atlas Research, N., et al., Comprehensive, Integrative Genomic Analysis of Diffuse Lower-Grade Gliomas. N Engl J Med, 2015. 372(26): p. 2481-98.

8. Ohgaki, H., Epidemiology of brain tumors. Methods Mol Biol, 2009. 472: p. 323-42.

9. Behin, A., et al., Primary brain tumours in adults. Lancet, 2003. 361(9354): p. 323-31.

10. Ohgaki, H. and P. Kleihues, Epidemiology and etiology of gliomas. Acta Neuropathol, 2005. 109(1): p. 93-108.

11. Furnari, F.B., et al., Malignant astrocytic glioma: genetics, biology, and paths to treatment. Genes Dev, 2007. 21(21): p. 2683-710.

12. van den Bent, M.J., Interobserver variation of the histopathological diagnosis in clinical trials on glioma: a clinician's perspective. Acta Neuropathol, 2010. 120(3): p. 297-304.

13. Ceccarelli, M., et al., Molecular Profiling Reveals Biologically Discrete Subsets and Pathways of Progression in Diffuse Glioma. Cell, 2016. 164(3): p. 550-63.

14. Nutt, C.L., et al., Gene expression-based classification of malignant gliomas correlates better with survival than histological classification. Cancer Res, 2003. 63(7): p. 1602-7.

15. Gravendeel, L.A., et al., Intrinsic gene expression profiles of gliomas are a better predictor of survival than histology. Cancer Res, 2009. 69(23): p. 9065-72.

16. Eckel-Passow, J.E., et al., Glioma Groups Based on 1p/19q, IDH, and TERT Promoter Mutations in Tumors. N Engl J Med, 2015. 372(26): p. 2499-508.

17. Suzuki, H., et al., Mutational landscape and clonal architecture in grade II and III gliomas. Nat Genet, 2015. 47(5): p. 458-68.

18. Jiao, Y., et al., Frequent ATRX, CIC, FUBP1 and IDH1 mutations refine the classification of malignant gliomas. Oncotarget, 2012. 3(7): p. 709-22.

19. Killela, P.J., et al., TERT promoter mutations occur frequently in gliomas and a subset of tumors derived from cells with low rates of self-renewal. Proc Natl Acad Sci U S A, 2013. 110(15): p. 6021-6.

20. Dubbink, H.J., et al., Molecular classification of anaplastic oligodendroglioma using nextgeneration sequencing: a report of the prospective randomized EORTC Brain Tumor Group 26951 phase III trial. Neuro Oncol, 2016. 18(3): p. 388-400.

21. Mellai, M., et al., Diagnostic revision of 206 adult gliomas (including 40 oligoastrocytomas) based on ATRX, IDH1/2 and 1p/19q status. J Neurooncol, 2017. 131(2): p. 213-222.

22. Gonda, D.D., et al., The Cancer Genome Atlas expression profiles of low-grade gliomas. Neurosurg Focus, 2014. 36(4): p. E23.

23. Ballester, L.Y., et al., Retrospective Analysis of Molecular and Immunohistochemical Characterization of 381 Primary Brain Tumors. J Neuropathol Exp Neurol, 2017. 76(3): p. 179188. 
24. Huse, J.T. and K.D. Aldape, The evolving role of molecular markers in the diagnosis and management of diffuse glioma. Clin Cancer Res, 2014. 20(22): p. 5601-11.

25. Lee, J., D.A. Solomon, and T. Tihan, The role of histone modifications and telomere alterations in the pathogenesis of diffuse gliomas in adults and children. J Neurooncol, 2017. 132(1): p. 1-11.

26. Paul, Y., et al., DNA methylation signatures for 2016 WHO classification subtypes of diffuse gliomas. Clin Epigenetics, 2017. 9: p. 32.

27. Hartmann, C. and A. von Deimling, Molecular pathology of oligodendroglial tumors. Recent Results Cancer Res, 2009. 171: p. 25-49.

28. Stark, A.M., et al., Acute Hydrocephalus due to Secondary Leptomeningeal Dissemination of an Anaplastic Oligodendroglioma. Case Rep Med, 2009. 2009: p. 370901.

29. Wheen, L.C., et al., Leptomeningeal infiltration as the presenting manifestation of a malignant glioma. J Clin Neurosci, 2006. 13(2): p. 298-301.

30. Debono, B., et al., Primary diffuse multinodular leptomeningeal gliomatosis: case report and review of the literature. Surg Neurol, 2006. 65(3): p. 273-82; discussion 282.

31. Verma, N., et al., Intramedullary spinal cord and leptomeningeal metastases from intracranial low-grade oligodendroglioma. Clin Imaging, 2014. 38(4): p. 505-7.

32. Joubert, C., et al., Solitary "aggressive" leptomeningeal anaplastic oligoastrocytoma. Rev Neurol (Paris), 2015. 171(11): p. 796-8.

33. Bourne, T.D., et al., Primary disseminated leptomeningeal oligodendroglioma with 1p deletion. Case report. J Neurosurg, 2006. 105(6 Suppl): p. 465-9.

34. Riva, M., et al., Primary leptomeningeal gliomatosis: case report and review of the literature. Neurol Sci, 2005. 26(2): p. 129-34.

35. Rogers, L.R., et al., Primary leptomeningeal oligodendroglioma: case report. Neurosurgery, 1995. 36(1): p. 166-8; discussion 169.

36. Chen, R., D.R. Macdonald, and D.A. Ramsay, Primary diffuse leptomeningeal oligodendroglioma. Case report. J Neurosurg, 1995. 83(4): p. 724-8.

37. Guo, Q., et al., Oligodendroglioma of the ciliary body: a unique case report and the review of literature. BMC Cancer, 2010. 10: p. 579.

38. Perry, A., et al., Oligodendroglial neoplasms with ganglioglioma-like maturation: a diagnostic pitfall. Acta Neuropathol, 2010. 120(2): p. 237-52.

39. Bromberg, J.E. and M.J. van den Bent, Oligodendrogliomas: molecular biology and treatment. Oncologist, 2009. 14(2): p. 155-63.

40. Johnson, D.R., et al., Genetically Defined Oligodendroglioma Is Characterized by Indistinct Tumor Borders at MRI. AJNR Am J Neuroradiol, 2017. 38(4): p. 678-684.

41. Van den Bent, M.J., et al., Oligodendroglioma. Crit Rev Oncol Hematol, 2008. 66(3): p. 262-72.

42. Mork, S.J., et al., Oligodendroglioma: incidence and biological behavior in a defined population. J Neurosurg, 1985. 63(6): p. 881-9.

43. Hacisalihoglu, P., et al., The Correlation Between $1 p / 19 q$ Codeletion, IDH1 Mutation, p53 Overexpression and Their Prognostic Roles in 41 Turkish Anaplastic Oligodendroglioma Patients. Turk Neurosurg, 2016.

44. Achey, R.L., et al., Incidence and survival trends in oligodendrogliomas and anaplastic oligodendrogliomas in the United States from 2000 to 2013: a CBTRUS Report. J Neurooncol, 2017.

45. van den Bent, M.J., et al., Chromosomal anomalies in oligodendroglial tumors are correlated with clinical features. Cancer, 2003. 97(5): p. 1276-84.

46. Jacob, R., et al., Oligodendroglioma: clinical profile and treatment results. Neurol India, 2002. 50(4): p. 462-6. 
47. Ellis, T.L., V.W. Stieber, and R.C. Austin, Oligodendroglioma. Curr Treat Options Oncol, 2003. 4(6): p. 479-90.

48. van den Bent, M.J., et al., Phase II study of first-line chemotherapy with temozolomide in recurrent oligodendroglial tumors: the European Organization for Research and Treatment of Cancer Brain Tumor Group Study 26971. J Clin Oncol, 2003. 21(13): p. 2525-8.

49. Hashimoto, N., et al., Correlation between genetic alteration and long-term clinical outcome of patients with oligodendroglial tumors, with identification of a consistent region of deletion on chromosome arm 1p. Cancer, 2003. 97(9): p. 2254-61.

50. Cairncross, J.G., et al., Specific genetic predictors of chemotherapeutic response and survival in patients with anaplastic oligodendrogliomas. J Natl Cancer Inst, 1998. 90(19): p. 1473-9.

51. Hagerstrand, D., et al., Gene expression analyses of grade II gliomas and identification of rPTPbeta/zeta as a candidate oligodendroglioma marker. Neuro Oncol, 2008. 10(1): p. 2-9.

52. Shaw, E.J., et al., Gene expression in oligodendroglial tumors. Anal Cell Pathol (Amst), 2010. 33(2): p. 81-94.

53. Landry, C.F., et al., Expression of oligodendrocytic mRNAs in glial tumors: changes associated with tumor grade and extent of neoplastic infiltration. Cancer Res, 1997. 57(18): p. 4098-104.

54. Lu, Q.R., et al., Sonic hedgehog--regulated oligodendrocyte lineage genes encoding $b H L H$ proteins in the mammalian central nervous system. Neuron, 2000. 25(2): p. 317-29.

55. Riemenschneider, M.J., T.H. Koy, and G. Reifenberger, Expression of oligodendrocyte lineage genes in oligodendroglial and astrocytic gliomas. Acta Neuropathol, 2004. 107(3): p. 277-82.

56. Hoang-Xuan, K., et al., OLIG-1 and 2 gene expression and oligodendroglial tumours. Neuropathol Appl Neurobiol, 2002. 28(2): p. 89-94.

57. Persson, A.I., et al., Non-stem cell origin for oligodendroglioma. Cancer Cell, 2010. 18(6): p. 66982.

58. Shoshan, Y., et al., Expression of oligodendrocyte progenitor cell antigens by gliomas: implications for the histogenesis of brain tumors. Proc Natl Acad Sci U S A, 1999. 96(18): p. 10361-6.

59. Briancon-Marjollet, A., et al., NG2-expressing glial precursor cells are a new potential oligodendroglioma cell initiating population in N-ethyl-N-nitrosourea-induced gliomagenesis. Carcinogenesis, 2010. 31(10): p. 1718-25.

60. Jarvela, S., et al., Carbonic anhydrase IX in oligodendroglial brain tumors. BMC Cancer, 2008. 8: p. 1.

61. Le Mercier, M., et al., Galectin 1 proangiogenic and promigratory effects in the Hs683 oligodendroglioma model are partly mediated through the control of BEX2 expression. Neoplasia, 2009. 11(5): p. 485-96.

62. Camby, I., et al., Galectins are differentially expressed in supratentorial pilocytic astrocytomas, astrocytomas, anaplastic astrocytomas and glioblastomas, and significantly modulate tumor astrocyte migration. Brain Pathol, 2001. 11(1): p. 12-26.

63. Le Mercier, M., et al., Galectins and gliomas. Brain Pathol, 2010. 20(1): p. 17-27.

64. Rorive, S., et al., Galectin-1 is highly expressed in human gliomas with relevance for modulation of invasion of tumor astrocytes into the brain parenchyma. Glia, 2001. 33(3): p. 241-55.

65. Le Mercier, M., et al., Evidence of galectin-1 involvement in glioma chemoresistance. Toxicol Appl Pharmacol, 2008. 229(2): p. 172-83.

66. Dai, C., et al., PDGF autocrine stimulation dedifferentiates cultured astrocytes and induces oligodendrogliomas and oligoastrocytomas from neural progenitors and astrocytes in vivo. Genes Dev, 2001. 15(15): p. 1913-25.

67. Sathornsumetee, S. and J.N. Rich, Antiangiogenic therapy in malignant glioma: promise and challenge. Curr Pharm Des, 2007. 13(35): p. 3545-58. 
68. Ferrer-Luna, R., et al., Whole-genomic survey of oligodendroglial tumors: correlation between allelic imbalances and gene expression profiles. J Neurooncol, 2011. 103(1): p. 71-85.

69. Shih, A.H. and E.C. Holland, Platelet-derived growth factor (PDGF) and glial tumorigenesis. Cancer Lett, 2006. 232(2): p. 139-47.

70. Shih, A.H., et al., Dose-dependent effects of platelet-derived growth factor-B on glial tumorigenesis. Cancer Res, 2004. 64(14): p. 4783-9.

71. Lokker, N.A., et al., Platelet-derived growth factor (PDGF) autocrine signaling regulates survival and mitogenic pathways in glioblastoma cells: evidence that the novel PDGF-C and PDGF-D ligands may play a role in the development of brain tumors. Cancer Res, 2002. 62(13): p. 372935.

72. Yan, H., et al., IDH1 and IDH2 mutations in gliomas. N Engl J Med, 2009. 360(8): p. 765-73.

73. Gravendeel, L.A., et al., Segregation of non-p.R132H mutations in IDH1 in distinct molecular subtypes of glioma. Hum Mutat, 2010. 31(3): p. E1186-99.

74. Lu, C., et al., IDH mutation impairs histone demethylation and results in a block to cell differentiation. Nature, 2012. 483(7390): p. 474-8.

75. Watanabe, T., et al., Deregulation of the TP53/p14ARF tumor suppressor pathway in low-grade diffuse astrocytomas and its influence on clinical course. Clin Cancer Res, 2003. 9(13): p. 488490.

76. Bettegowda, C., et al., Mutations in CIC and FUBP1 contribute to human oligodendroglioma. Science, 2011. 333(6048): p. 1453-5.

77. Cahill, D.P., D.N. Louis, and J.G. Cairncross, Molecular background of oligodendroglioma: $1 p / 19 q$, IDH, TERT, CIC and FUBP1. CNS Oncol, 2015. 4(5): p. 287-94.

78. Pekmezci, M., et al., Adult infiltrating gliomas with WHO 2016 integrated diagnosis: additional prognostic roles of ATRX and TERT. Acta Neuropathol, 2017.

79. Sahm, F., et al., CIC and FUBP1 mutations in oligodendrogliomas, oligoastrocytomas and astrocytomas. Acta Neuropathol, 2012. 123(6): p. 853-60.

80. Chittaranjan, S., et al., Mutations in $\mathrm{CIC}$ and IDH1 cooperatively regulate 2-hydroxyglutarate levels and cell clonogenicity. Oncotarget, 2014. 5(17): p. 7960-79.

81. Klink, B., et al., A novel, diffusely infiltrative xenograft model of human anaplastic oligodendroglioma with mutations in FUBP1, CIC, and IDH1. PLoS One, 2013. 8(3): p. e59773.

82. Yip, S., et al., Concurrent CIC mutations, IDH mutations, and $1 p / 19 q$ loss distinguish oligodendrogliomas from other cancers. J Pathol, 2012. 226(1): p. 7-16.

83. Van Den Bent, M.J., J.E. Bromberg, and J. Buckner, Low-grade and anaplastic oligodendroglioma. Handb Clin Neurol, 2016. 134: p. 361-80.

84. Sasaki, H., et al., PTEN is a target of chromosome 10q loss in anaplastic oligodendrogliomas and PTEN alterations are associated with poor prognosis. Am J Pathol, 2001. 159(1): p. 359-67.

85. Wiencke, J.K., et al., Methylation of the PTEN promoter defines low-grade gliomas and secondary glioblastoma. Neuro Oncol, 2007. 9(3): p. 271-9.

86. Mollemann, M., et al., Frequent promoter hypermethylation and low expression of the MGMT gene in oligodendroglial tumors. Int J Cancer, 2005. 113(3): p. 379-85.

87. Watanabe, T., et al., Promoter hypermethylation and homozygous deletion of the p14ARF and p16INK4a genes in oligodendrogliomas. Acta Neuropathol, 2001. 101(3): p. 185-9.

88. Wolter, M., et al., Oligodendroglial tumors frequently demonstrate hypermethylation of the CDKN2A (MTS1, p16INK4a), p14ARF, and CDKN2B (MTS2, p15INK4b) tumor suppressor genes. J Neuropathol Exp Neurol, 2001. 60(12): p. 1170-80.

89. Alonso, M.E., et al., Aberrant promoter methylation of multiple genes in oligodendrogliomas and ependymomas. Cancer Genet Cytogenet, 2003. 144(2): p. 134-42. 
90. Dong, S.M., et al., Concurrent hypermethylation of multiple genes is associated with grade of oligodendroglial tumors. J Neuropathol Exp Neurol, 2001. 60(8): p. 808-16.

91. Gonzalez-Gomez, P., et al., CpG island methylation status and mutation analysis of the RB1 gene essential promoter region and protein-binding pocket domain in nervous system tumours. $\mathrm{Br} \mathrm{J}$ Cancer, 2003. 88(1): p. 109-14.

92. Bortolotto, S., et al., CDKN2A/p16 inactivation in the prognosis of oligodendrogliomas. Int J Cancer, 2000. 88(4): p. 554-7.

93. Kuo, L.T., et al., Multiplexed methylation profiles of tumor suppressor genes and clinical outcome in oligodendroglial tumors. Cancer Med, 2016. 5(8): p. 1830-9.

94. Mulholland, S., et al., MGMT CPG island is invariably methylated in adult astrocytic and oligodendroglial tumors with IDH1 or IDH2 mutations. Int J Cancer, 2012. 131(5): p. 1104-13.

95. Lhotska, H., et al., Genetic and epigenetic characterization of low-grade gliomas reveals frequent methylation of the MLH3 gene. Genes Chromosomes Cancer, 2015. 54(11): p. 655-67.

96. Brandes, A.A., et al., Correlations between O6-methylguanine DNA methyltransferase promoter methylation status, $1 p$ and $19 q$ deletions, and response to temozolomide in anaplastic and recurrent oligodendroglioma: a prospective GICNO study. J Clin Oncol, 2006. 24(29): p. 4746-53.

97. Lavon, I., et al., Longitudinal assessment of genetic and epigenetic markers in oligodendrogliomas. Clin Cancer Res, 2007. 13(5): p. 1429-37.

98. McTyre, E., et al., Outcomes for Anaplastic Glioma Treated With Radiation Therapy With or Without Concurrent Temozolomide. Am J Clin Oncol, 2017.

99. Tanaka, K., et al., Combined IDH1 mutation and MGMT methylation status on long-term survival of patients with cerebral low-grade glioma. Clin Neurol Neurosurg, 2015. 138: p. 37-44.

100. Nguyen, S.A., et al., Novel MSH6 mutations in treatment-naive glioblastoma and anaplastic oligodendroglioma contribute to temozolomide resistance independently of MGMT promoter methylation. Clin Cancer Res, 2014. 20(18): p. 4894-903.

101. Holdhoff, M., et al., Genetic landscape of extreme responders with anaplastic oligodendroglioma. Oncotarget, 2017.

102. Shin, J.Y. and A.Z. Diaz, Utilization and impact of adjuvant therapy in anaplastic oligodendroglioma: an analysis on 1692 patients. J Neurooncol, 2016. 129(3): p. 567-75.

103. Shin, J.Y., J.K. Yoon, and A.Z. Diaz, Racial disparities in anaplastic oligodendroglioma: An analysis on 1643 patients. J Clin Neurosci, 2017. 37: p. 34-39.

104. Kunitz, A., et al., DNA hypermethylation and aberrant expression of the EMP3 gene at $19 q 13.3$ in Human Gliomas. Brain Pathol, 2007. 17(4): p. 363-70.

105. Li, K.K., et al., EMP3 overexpression is associated with oligodendroglial tumors retaining chromosome arms 1p and 19q. Int J Cancer, 2007. 120(4): p. 947-50.

106. Pasini, A., et al., $\mathrm{LOH} 19 q$ indicates shorter disease progression-free interval in low-grade oligodendrogliomas with EMP3 methylation. Oncol Rep, 2012. 28(6): p. 2271-7.

107. Blough, M.D., et al., DNA hypermethylation and 1p Loss silence NHE-1 in oligodendroglioma. Ann Neurol, 2012. 71(6): p. 845-9.

108. Mur, P., et al., Codeletion of $1 p$ and $19 q$ determines distinct gene methylation and expression profiles in IDH-mutated oligodendroglial tumors. Acta Neuropathol, 2013. 126(2): p. 277-89.

109. Noushmehr, $\mathrm{H}$., et al., Identification of a $\mathrm{CpG}$ island methylator phenotype that defines a distinct subgroup of glioma. Cancer Cell, 2010. 17(5): p. 510-22.

110. Sahm, F., et al., Analysis of CIC-associated CpG island methylation in oligoastrocytoma. Neuropathol Appl Neurobiol, 2013. 39(7): p. 831-6.

111. van den Bent, M.J., et al., A hypermethylated phenotype is a better predictor of survival than MGMT methylation in anaplastic oligodendroglial brain tumors: a report from EORTC study 26951. Clin Cancer Res, 2011. 17(22): p. 7148-55. 
112. Barbashina, V., et al., Allelic losses at $1 p 36$ and $19 q 13$ in gliomas: correlation with histologic classification, definition of a 150-kb minimal deleted region on 1p36, and evaluation of CAMTA1 as a candidate tumor suppressor gene. Clin Cancer Res, 2005. 11(3): p. 1119-28.

113. Reifenberger, J., et al., Molecular genetic analysis of oligodendroglial tumors shows preferential allelic deletions on 19q and 1p. Am J Pathol, 1994. 145(5): p. 1175-90.

114. Maintz, D., et al., Molecular genetic evidence for subtypes of oligoastrocytomas. J Neuropathol Exp Neurol, 1997. 56(10): p. 1098-104.

115. Riemenschneider, M.J., J. Reifenberger, and G. Reifenberger, Frequent biallelic inactivation and transcriptional silencing of the DIRAS3 gene at $1 p 31$ in oligodendroglial tumors with $1 p$ loss. Int J Cancer, 2008. 122(11): p. 2503-10.

116. Griffin, C.A., et al., Identification of $\operatorname{der}(1 ; 19)(q 10 ; p 10)$ in five oligodendrogliomas suggests mechanism of concurrent 1p and 19q loss. J Neuropathol Exp Neurol, 2006. 65(10): p. 988-94.

117. Jenkins, R.B., et al., A t(1;19)(q10;p10) mediates the combined deletions of $1 p$ and $19 q$ and predicts a better prognosis of patients with oligodendroglioma. Cancer Res, 2006. 66(20): p. 9852-61.

118. Hinrichs, B.H., et al., Farewell to GBM-O: Genomic and transcriptomic profiling of glioblastoma with oligodendroglioma component reveals distinct molecular subgroups. Acta Neuropathol Commun, 2016. 4: p. 4.

119. Kanoto, M., et al., Radiological imaging features of glioblastoma with oligodendroglioma component: a comparison with conventional glioblastoma. Acta Radiol Open, 2016. 5(11): p. 2058460116675191.

120. Yan, H., et al., Mutant metabolic enzymes are at the origin of gliomas. Cancer Res, 2009. 69(24): p. 9157-9.

121. Pohl, U., J.G. Cairncross, and D.N. Louis, Homozygous deletions of the CDKN2C/p18INK4C gene on the short arm of chromosome 1 in anaplastic oligodendrogliomas. Brain Pathol, 1999. 9(4): p. 639-43.

122. Smits, M., Imaging of oligodendroglioma. Br J Radiol, 2016. 89(1060): p. 20150857.

123. Hatanpaa, K.J., et al., Molecular diagnosis of oligodendroglioma in paraffin sections. Lab Invest, 2003. 83(3): p. 419-28.

124. Pinkham, M.B., et al., FISHing Tips: What Every Clinician Should Know About 1p19q Analysis in Gliomas Using Fluorescence in situ Hybridisation. Clin Oncol (R Coll Radiol), 2015. 27(8): p. 44553.

125. Felsberg, J., et al., Oligodendroglial tumors: refinement of candidate regions on chromosome arm 1p and correlation of 1p/19q status with survival. Brain Pathol, 2004. 14(2): p. 121-30.

126. Bello, M.J., et al., Allelic loss at $1 p$ and $19 q$ frequently occurs in association and may represent early oncogenic events in oligodendroglial tumors. Int J Cancer, 1995. 64(3): p. 207-10.

127. Reifenberger, G. and D.N. Louis, Oligodendroglioma: toward molecular definitions in diagnostic neuro-oncology. J Neuropathol Exp Neurol, 2003. 62(2): p. 111-26.

128. Yip, S., A.J. lafrate, and D.N. Louis, Molecular diagnostic testing in malignant gliomas: a practical update on predictive markers. J Neuropathol Exp Neurol, 2008. 67(1): p. 1-15.

129. Smith, J.S., et al., Alterations of chromosome arms $1 p$ and $19 q$ as predictors of survival in oligodendrogliomas, astrocytomas, and mixed oligoastrocytomas. J Clin Oncol, 2000. 18(3): p. 636-45.

130. Polivka, J., Jr., et al., Co-deletion of $1 p / 19 q$ as Prognostic and Predictive Biomarker for Patients in West Bohemia with Anaplastic Oligodendroglioma. Anticancer Res, 2016. 36(1): p. 471-6.

131. Jiang, H., et al., Tumor cell-specific chromosomal abnormality in the vascular endothelial cells of anaplastic oligodendroglioma. J Neurosurg, 2016. 125(4): p. 995-1001. 
132. Speirs, C.K., et al., Impact of $1 p / 19 q$ codeletion and histology on outcomes of anaplastic gliomas treated with radiation therapy and temozolomide. Int J Radiat Oncol Biol Phys, 2015. 91(2): p. 268-76.

133. Kros, J.M., et al., Panel review of anaplastic oligodendroglioma from European Organization For Research and Treatment of Cancer Trial 26951: assessment of consensus in diagnosis, influence of 1p/19q loss, and correlations with outcome. J Neuropathol Exp Neurol, 2007. 66(6): p. 545-51.

134. van den Bent, M.J., et al., Adjuvant procarbazine, lomustine, and vincristine improves progression-free survival but not overall survival in newly diagnosed anaplastic oligodendrogliomas and oligoastrocytomas: a randomized European Organisation for Research and Treatment of Cancer phase III trial. J Clin Oncol, 2006. 24(18): p. 2715-22.

135. Lassman, A.B., et al., International retrospective study of over 1000 adults with anaplastic oligodendroglial tumors. Neuro Oncol, 2011. 13(6): p. 649-59.

136. Jaeckle, K.A., et al., Current strategies in treatment of oligodendroglioma: evolution of molecular signatures of response. J Clin Oncol, 2006. 24(8): p. 1246-52.

137. Burger, P.C., What is an oligodendroglioma? Brain Pathol, 2002. 12(2): p. 257-9.

138. Intergroup Radiation Therapy Oncology Group, T., et al., Phase III trial of chemotherapy plus radiotherapy compared with radiotherapy alone for pure and mixed anaplastic oligodendroglioma: Intergroup Radiation Therapy Oncology Group Trial 9402. J Clin Oncol, 2006. 24(18): p. 2707-14.

139. Everhard, S., et al., MGMT methylation: a marker of response to temozolomide in low-grade gliomas. Ann Neurol, 2006. 60(6): p. 740-3.

140. Aihara, K., et al., Genetic and epigenetic stability of oligodendrogliomas at recurrence. Acta Neuropathol Commun, 2017. 5(1): p. 18.

141. Gerstein, M.B., et al., Integrative analysis of the Caenorhabditis elegans genome by the modENCODE project. Science, 2010. 330(6012): p. 1775-87.

142. Consortium, E.P., The ENCODE (ENCyclopedia Of DNA Elements) Project. Science, 2004. 306(5696): p. 636-40.

143. Niu, D.K. and L. Jiang, Can ENCODE tell us how much junk DNA we carry in our genome? Biochem Biophys Res Commun, 2013. 430(4): p. 1340-3.

144. Doolittle, W.F., Is junk DNA bunk? A critique of ENCODE. Proc Natl Acad Sci U S A, 2013. 110(14): p. 5294-300.

145. Consortium, E.P., A user's guide to the encyclopedia of DNA elements (ENCODE). PLoS Biol, 2011. 9(4): p. e1001046.

146. Sloan, C.A., et al., ENCODE data at the ENCODE portal. Nucleic Acids Res, 2016. 44(D1): p. D72632.

147. Yue, F., et al., A comparative encyclopedia of DNA elements in the mouse genome. Nature, 2014. 515(7527): p. 355-64.

148. Consortium, E.P., et al., Identification and analysis of functional elements in $1 \%$ of the human genome by the ENCODE pilot project. Nature, 2007. 447(7146): p. 799-816.

149. Consortium, E.P., An integrated encyclopedia of DNA elements in the human genome. Nature, 2012. 489(7414): p. 57-74.

150. Qu, H. and X. Fang, A brief review on the Human Encyclopedia of DNA Elements (ENCODE) project. Genomics Proteomics Bioinformatics, 2013. 11(3): p. 135-41.

151. Ender, C., et al., A human snoRNA with microRNA-like functions. Mol Cell, 2008. 32(4): p. 519-28.

152. Esteller, M., Non-coding RNAs in human disease. Nat Rev Genet, 2011. 12(12): p. 861-74.

153. Diederichs, S., Non-coding RNA and disease. RNA Biol, 2012. 9(6): p. 701-2.

154. Chen, G., et al., LncRNADisease: a database for long-non-coding RNA-associated diseases. Nucleic Acids Res, 2013. 41(Database issue): p. D983-6. 
155. Bejerano, G., et al., Ultraconserved elements in the human genome. Science, 2004. 304(5675): $p$. 1321-5.

156. Calin, G.A., et al., Ultraconserved regions encoding ncRNAs are altered in human leukemias and carcinomas. Cancer Cell, 2007. 12(3): p. 215-29.

157. Calin, G.A., et al., Human microRNA genes are frequently located at fragile sites and genomic regions involved in cancers. Proc Natl Acad Sci U S A, 2004. 101(9): p. 2999-3004.

158. Ambros, V., The functions of animal microRNAs. Nature, 2004. 431(7006): p. 350-5.

159. Sevignani, C., et al., Mammalian microRNAs: a small world for fine-tuning gene expression. Mamm Genome, 2006. 17(3): p. 189-202.

160. Doench, J.G. and P.A. Sharp, Specificity of microRNA target selection in translational repression. Genes Dev, 2004. 18(5): p. 504-11.

161. Zeng, Y., R. Yi, and B.R. Cullen, MicroRNAs and small interfering RNAs can inhibit mRNA expression by similar mechanisms. Proc Natl Acad Sci U S A, 2003. 100(17): p. 9779-84.

162. Filipowicz, W., S.N. Bhattacharyya, and N. Sonenberg, Mechanisms of post-transcriptional regulation by microRNAs: are the answers in sight? Nat Rev Genet, 2008. 9(2): p. 102-14.

163. Reczko, M., et al., Accurate microRNA Target Prediction Using Detailed Binding Site Accessibility and Machine Learning on Proteomics Data. Front Genet, 2011. 2: p. 103.

164. Lewis, B.P., et al., Prediction of mammalian microRNA targets. Cell, 2003. 115(7): p. 787-98.

165. Yekta, S., I.H. Shih, and D.P. Bartel, MicroRNA-directed cleavage of HOXB8 mRNA. Science, 2004. 304(5670): p. 594-6.

166. Lee, R.C., R.L. Feinbaum, and V. Ambros, The C. elegans heterochronic gene lin-4 encodes small RNAs with antisense complementarity to lin-14. Cell, 1993. 75(5): p. 843-54.

167. Lee, R.C. and V. Ambros, An extensive class of small RNAs in Caenorhabditis elegans. Science, 2001. 294(5543): p. 862-4.

168. Ambros, V., et al., MicroRNAs and other tiny endogenous RNAs in C. elegans. Curr Biol, 2003. 13(10): p. 807-18.

169. Bartel, D.P., MicroRNAs: genomics, biogenesis, mechanism, and function. Cell, 2004. 116(2): p. 281-97.

170. Pasquinelli, A.E. and G. Ruvkun, Control of developmental timing by micrornas and their targets. Annu Rev Cell Dev Biol, 2002. 18: p. 495-513.

171. Lim, L.P., et al., The microRNAs of Caenorhabditis elegans. Genes Dev, 2003. 17(8): p. 991-1008.

172. Carrington, J.C. and V. Ambros, Role of microRNAs in plant and animal development. Science, 2003. 301(5631): p. 336-8.

173. Ambros, V., MicroRNA pathways in flies and worms: growth, death, fat, stress, and timing. Cell, 2003. 113(6): p. 673-6.

174. Grosshans, H. and W. Filipowicz, Proteomics joins the search for microRNA targets. Cell, 2008. 134(4): p. 560-2.

175. Sempere, L.F., et al., Expression profiling of mammalian microRNAs uncovers a subset of brainexpressed microRNAs with possible roles in murine and human neuronal differentiation. Genome Biol, 2004. 5(3): p. R13.

176. Kim, V.N., J. Han, and M.C. Siomi, Biogenesis of small RNAs in animals. Nat Rev Mol Cell Biol, 2009. 10(2): p. 126-39.

177. Lee, Y., et al., The nuclear RNase III Drosha initiates microRNA processing. Nature, 2003. 425(6956): p. 415-9.

178. Lee, Y., et al., MicroRNA maturation: stepwise processing and subcellular localization. EMBO J, 2002. 21(17): p. 4663-70.

179. Lund, E., et al., Nuclear export of microRNA precursors. Science, 2004. 303(5654): p. 95-8. 
180. Yi, R., et al., Exportin-5 mediates the nuclear export of pre-microRNAs and short hairpin RNAs. Genes Dev, 2003. 17(24): p. 3011-6.

181. Winter, J., et al., Many roads to maturity: microRNA biogenesis pathways and their regulation. Nat Cell Biol, 2009. 11(3): p. 228-34.

182. Pang, J.C., et al., Oncogenic role of microRNAs in brain tumors. Acta Neuropathol, 2009. 117(6): p. 599-611.

183. Croce, C.M., Oncogenes and cancer. N Engl J Med, 2008. 358(5): p. 502-11.

184. Croce, C.M., Causes and consequences of microRNA dysregulation in cancer. Nat Rev Genet, 2009. 10(10): p. 704-14.

185. Calin, G.A., et al., Frequent deletions and down-regulation of micro- RNA genes miR15 and miR16 at $13 q 14$ in chronic lymphocytic leukemia. Proc Natl Acad Sci U S A, 2002. 99(24): p. 15524-9.

186. Calin, G.A., et al., MicroRNA profiling reveals distinct signatures in B cell chronic lymphocytic leukemias. Proc Natl Acad Sci U S A, 2004. 101(32): p. 11755-60.

187. Calin, G.A., et al., A MicroRNA signature associated with prognosis and progression in chronic lymphocytic leukemia. N Engl J Med, 2005. 353(17): p. 1793-801.

188. Tsuda, N., et al., Synthetic microRNA and double-stranded RNA targeting the 3'-untranslated region of HER-2/neu MRNA inhibit HER-2 protein expression in ovarian cancer cells. Int J Oncol, 2005. 27(5): p. 1299-306.

189. Gatti, J.L., et al., Identification, proteomic profiling, and origin of ram epididymal fluid exosomelike vesicles. Biol Reprod, 2005. 72(6): p. 1452-65.

190. Murakami, Y., et al., Comprehensive analysis of microRNA expression patterns in hepatocellular carcinoma and non-tumorous tissues. Oncogene, 2006. 25(17): p. 2537-45.

191. Hwang, H.W. and J.T. Mendell, MicroRNAs in cell proliferation, cell death, and tumorigenesis. $\mathrm{Br}$ J Cancer, 2006. 94(6): p. 776-80.

192. Esquela-Kerscher, A. and F.J. Slack, Oncomirs - microRNAs with a role in cancer. Nat Rev Cancer, 2006. 6(4): p. 259-69.

193. Dalmay, T. and D.R. Edwards, MicroRNAs and the hallmarks of cancer. Oncogene, 2006. 25(46): p. 6170-5.

194. Ventura, A. and T. Jacks, MicroRNAs and cancer: short RNAs go a long way. Cell, 2009. 136(4): p. 586-91.

195. Chen, C.Z., MicroRNAs as oncogenes and tumor suppressors. N Engl J Med, 2005. 353(17): p. 1768-71.

196. Iorio, M.V., et al., MicroRNA gene expression deregulation in human breast cancer. Cancer Res, 2005. 65(16): p. 7065-70.

197. Hayashita, Y., et al., A polycistronic microRNA cluster, miR-17-92, is overexpressed in human lung cancers and enhances cell proliferation. Cancer Res, 2005. 65(21): p. 9628-32.

198. Michael, M.Z., et al., Reduced accumulation of specific microRNAs in colorectal neoplasia. Mol Cancer Res, 2003. 1(12): p. 882-91.

199. Takamizawa, J., et al., Reduced expression of the let-7 microRNAs in human lung cancers in association with shortened postoperative survival. Cancer Res, 2004. 64(11): p. 3753-6.

200. Chan, J.A., A.M. Krichevsky, and K.S. Kosik, MicroRNA-21 is an antiapoptotic factor in human glioblastoma cells. Cancer Res, 2005. 65(14): p. 6029-33.

201. Garzon, R., et al., MicroRNA expression and function in cancer. Trends Mol Med, 2006. 12(12): $\mathrm{p}$. 580-7.

202. Zhang, L., et al., microRNAs exhibit high frequency genomic alterations in human cancer. Proc Natl Acad Sci U S A, 2006. 103(24): p. 9136-41. 
203. Babashah, S. and M. Soleimani, The oncogenic and tumour suppressive roles of microRNAs in cancer and apoptosis. Eur J Cancer, 2011. 47(8): p. 1127-37.

204. Volinia, S., et al., A microRNA expression signature of human solid tumors defines cancer gene targets. Proc Natl Acad Sci U S A, 2006. 103(7): p. 2257-61.

205. Hauptman, N. and D. Glavac, Long non-coding RNA in cancer. Int J Mol Sci, 2013. 14(3): p. 465569.

206. Gupta, R.A., et al., Long non-coding RNA HOTAIR reprograms chromatin state to promote cancer metastasis. Nature, 2010. 464(7291): p. 1071-6.

207. Gibb, E.A., C.J. Brown, and W.L. Lam, The functional role of long non-coding RNA in human carcinomas. Mol Cancer, 2011. 10: p. 38.

208. Gutschner, T. and S. Diederichs, The hallmarks of cancer: a long non-coding RNA point of view. RNA Biol, 2012. 9(6): p. 703-19.

209. Qi, P. and X. Du, The long non-coding RNAs, a new cancer diagnostic and therapeutic gold mine. Mod Pathol, 2013. 26(2): p. 155-65.

210. Huarte, M. and J.L. Rinn, Large non-coding RNAs: missing links in cancer? Hum Mol Genet, 2010. 19(R2): p. R152-61.

211. Cheetham, S.W., et al., Long noncoding RNAs and the genetics of cancer. Br J Cancer, 2013. 108(12): p. 2419-25.

212. Rinn, J.L., et al., Functional demarcation of active and silent chromatin domains in human HOX loci by noncoding RNAs. Cell, 2007. 129(7): p. 1311-23.

213. Dinger, M.E., et al., Differentiating protein-coding and noncoding RNA: challenges and ambiguities. PLoS Comput Biol, 2008. 4(11): p. e1000176.

214. Guenzl, P.M. and D.P. Barlow, Macro IncRNAs: a new layer of cis-regulatory information in the mammalian genome. RNA Biol, 2012. 9(6): p. 731-41.

215. Maicher, A., L. Kastner, and B. Luke, Telomeres and disease: enter TERRA. RNA Biol, 2012. 9(6): p. 843-9.

216. Calin, G.A. and C.M. Croce, MicroRNA signatures in human cancers. Nat Rev Cancer, 2006. 6(11): p. 857-66.

217. Lu, J., et al., MicroRNA expression profiles classify human cancers. Nature, 2005. 435(7043): p. 834-8.

218. Bottoni, A., et al., Identification of differentially expressed microRNAs by microarray: a possible role for microRNA genes in pituitary adenomas. J Cell Physiol, 2007. 210(2): p. 370-7.

219. Cao, X., et al., Noncoding RNAs in the mammalian central nervous system. Annu Rev Neurosci, 2006. 29: p. 77-103.

220. Fiore, R., et al., Mef2-mediated transcription of the miR379-410 cluster regulates activitydependent dendritogenesis by fine-tuning Pumilio2 protein levels. EMBO J, 2009. 28(6): p. 697710.

221. Lagos-Quintana, M., et al., Identification of tissue-specific microRNAs from mouse. Curr Biol, 2002. 12(9): p. 735-9.

222. Smirnova, L., et al., Regulation of miRNA expression during neural cell specification. Eur J Neurosci, 2005. 21(6): p. 1469-77.

223. Yokota, T., [MicroRNA and central nervous system]. Brain Nerve, 2009. 61(2): p. 167-76.

224. Vo, N., et al., A cAMP-response element binding protein-induced microRNA regulates neuronal morphogenesis. Proc Natl Acad Sci U S A, 2005. 102(45): p. 16426-31.

225. Schratt, G.M., et al., A brain-specific microRNA regulates dendritic spine development. Nature, 2006. 439(7074): p. 283-9.

226. Malzkorn, B., et al., Identification and functional characterization of microRNAs involved in the malignant progression of gliomas. Brain Pathol, 2010. 20(3): p. 539-50. 
227. Ciafre, S.A., et al., Extensive modulation of a set of microRNAs in primary glioblastoma. Biochem Biophys Res Commun, 2005. 334(4): p. 1351-8.

228. Lawler, S. and E.A. Chiocca, Emerging functions of microRNAs in glioblastoma. J Neurooncol, 2009. 92(3): p. 297-306.

229. Palumbo, S., et al., Emerging roles of microRNA in modulating cell-death processes in malignant glioma. J Cell Physiol, 2014. 229(3): p. 277-86.

230. Silber, J., C.D. James, and J.G. Hodgson, microRNAs in gliomas: small regulators of a big problem. Neuromolecular Med, 2009. 11(3): p. 208-22.

231. Papagiannakopoulos, T., A. Shapiro, and K.S. Kosik, MicroRNA-21 targets a network of key tumor-suppressive pathways in glioblastoma cells. Cancer Res, 2008. 68(19): p. 8164-72.

232. Gabriely, G., et al., MicroRNA 21 promotes glioma invasion by targeting matrix metalloproteinase regulators. Mol Cell Biol, 2008. 28(17): p. 5369-80.

233. Moore, L.M. and W. Zhang, Targeting miR-21 in glioma: a small RNA with big potential. Expert Opin Ther Targets, 2010. 14(11): p. 1247-57.

234. Wong, S.T., et al., MicroRNA-21 inhibition enhances in vitro chemosensitivity of temozolomideresistant glioblastoma cells. Anticancer Res, 2012. 32(7): p. 2835-41.

235. Ilhan-Mutlu, A., et al., Plasma MicroRNA-21 concentration may be a useful biomarker in glioblastoma patients. Cancer Invest, 2012. 30(8): p. 615-21.

236. Polajeva, J., et al., miRNA-21 is developmentally regulated in mouse brain and is co-expressed with SOX2 in glioma. BMC Cancer, 2012. 12: p. 378.

237. Li, C., et al., Prognostic role of microRNA-21 expression in gliomas: a meta-analysis. J Neurooncol, 2016. 130(1): p. 11-17.

238. He, X.Y., et al., Prognostic Role of microRNA-21 Expression in Brain Tumors: a Meta-analysis. Mol Neurobiol, 2016. 53(3): p. 1856-61.

239. Hermansen, S.K., et al., miR-21 Is Linked to Glioma Angiogenesis: A Co-Localization Study. J Histochem Cytochem, 2016. 64(2): p. 138-48.

240. Turner, J.D., et al., The many roles of microRNAs in brain tumor biology. Neurosurg Focus, 2010. 28(1): p. E3.

241. Asadi-Moghaddam, K., E.A. Chiocca, and S.E. Lawler, Potential role of miRNAs and their inhibitors in glioma treatment. Expert Rev Anticancer Ther, 2010. 10(11): p. 1753-62.

242. Gabriely, G., et al., Human glioma growth is controlled by microRNA-10b. Cancer Res, 2011. 71(10): p. 3563-72.

243. Mei, J., R. Bachoo, and C.L. Zhang, MicroRNA-146a inhibits glioma development by targeting Notch1. Mol Cell Biol, 2011. 31(17): p. 3584-92.

244. Wang, Y., et al., MicroRNAs involved in the EGFR/PTEN/AKT pathway in gliomas. J Neurooncol, 2012. 106(2): p. 217-24.

245. Zhang, Y., A. Dutta, and R. Abounader, The role of microRNAs in glioma initiation and progression. Front Biosci (Landmark Ed), 2012. 17: p. 700-12.

246. Ouyang, Q., et al., MicroRNAs and cell cycle of malignant glioma. Int J Neurosci, 2016. 126(1): p. 1-9.

247. Moser, J.J. and M.J. Fritzler, The microRNA and messengerRNA profile of the RNA-induced silencing complex in human primary astrocyte and astrocytoma cells. PLoS One, 2010. 5(10): p. e13445.

248. Auffinger, B., et al., MicroRNA targeting as a therapeutic strategy against glioma. Curr Mol Med, 2013. 13(4): p. 535-42.

249. Short, S.C., Science in Focus: MicroRNA in Glioma - Potential as Biomarkers and Therapeutic Targets. Clin Oncol (R Coll Radiol), 2016. 28(9): p. 543-6. 
250. Rolle, K., miRNA Multiplayers in glioma. From bench to bedside. Acta Biochim Pol, 2015. 62(3): $\mathrm{p}$. 353-65.

251. Nicoloso, M.S. and G.A. Calin, MicroRNA involvement in brain tumors: from bench to bedside. Brain Pathol, 2008. 18(1): p. 122-9.

252. Hummel, R., J. Maurer, and J. Haier, MicroRNAs in brain tumors : a new diagnostic and therapeutic perspective? Mol Neurobiol, 2011. 44(3): p. 223-34.

253. Baraniskin, A., et al., Identification of microRNAs in the cerebrospinal fluid as biomarker for the diagnosis of glioma. Neuro Oncol, 2012. 14(1): p. 29-33.

254. Yu, X. and Z. Li, Serum microRNAs as potential noninvasive biomarkers for glioma. Tumour Biol, 2016. 37(2): p. 1407-10.

255. Areeb, Z., et al., MicroRNA as potential biomarkers in Glioblastoma. J Neurooncol, 2015. 125(2): p. 237-48.

256. Nelson, P.T., et al., RAKE and LNA-ISH reveal microRNA expression and localization in archival human brain. RNA, 2006. 12(2): p. 187-91.

257. Silber, J., et al., miR-124 and miR-137 inhibit proliferation of glioblastoma multiforme cells and induce differentiation of brain tumor stem cells. BMC Med, 2008. 6: p. 14.

258. Li, K.K., et al., MIR-137 suppresses growth and invasion, is downregulated in oligodendroglial tumors and targets CSE1L. Brain Pathol, 2013. 23(4): p. 426-39.

259. Lages, E., et al., MicroRNA and target protein patterns reveal physiopathological features of glioma subtypes. PLoS One, 2011. 6(5): p. e20600.

260. Lau, P., et al., Identification of dynamically regulated microRNA and mRNA networks in developing oligodendrocytes. J Neurosci, 2008. 28(45): p. 11720-30.

261. Lai, N., et al., Differential expression of microRNA-210 in gliomas of variable cell origin and correlation between increased expression levels and disease progression in astrocytic tumours. Folia Neuropathol, 2014. 52(1): p. 79-85.

262. Li, A., et al., Unsupervised analysis of transcriptomic profiles reveals six glioma subtypes. Cancer Res, 2009. 69(5): p. 2091-9.

263. Amaral, F.C., et al., MicroRNAs differentially expressed in ACTH-secreting pituitary tumors. J Clin Endocrinol Metab, 2009. 94(1): p. 320-3.

264. Valente, V., et al., Selection of suitable housekeeping genes for expression analysis in glioblastoma using quantitative RT-PCR. BMC Mol Biol, 2009. 10: p. 17.

265. Valente, V., et al., Selection of suitable housekeeping genes for expression analysis in glioblastoma using quantitative RT-PCR. Ann Neurosci, 2014. 21(2): p. 62-3.

266. Saldanha-Araujo, F., et al., Cancer/Testis antigen expression on mesenchymal stem cells isolated from different tissues. Anticancer Res, 2010. 30(12): p. 5023-7.

267. Betel, D., et al., Comprehensive modeling of microRNA targets predicts functional non-conserved and non-canonical sites. Genome Biol, 2010. 11(8): p. R90.

268. Cortez, M.A., et al., miR-29b and miR-125a regulate podoplanin and suppress invasion in glioblastoma. Genes Chromosomes Cancer, 2010. 49(11): p. 981-90.

269. Chen, X., F.J. Slack, and H. Zhao, Joint analysis of expression profiles from multiple cancers improves the identification of microRNA-gene interactions. Bioinformatics, 2013. 29(17): p. 2137-45.

270. Riemenschneider, M.J., et al., Molecular diagnostics of gliomas: state of the art. Acta Neuropathol, 2010. 120(5): p. 567-84.

271. Qin, L.Y., et al., The CCND1 G870A gene polymorphism and brain tumor risk: a meta-analysis. Asian Pac J Cancer Prev, 2014. 15(8): p. 3607-12.

272. Zong, H., et al., Association between the G870A polymorphism of Cyclin D1 gene and glioma risk. Tumour Biol, 2014. 35(8): p. 8095-101. 
273. LeBoeuf, M., et al., Hdac1 and Hdac2 act redundantly to control p63 and p53 functions in epidermal progenitor cells. Dev Cell, 2010. 19(6): p. 807-18.

274. Annunziata, M., et al., Somatostatin and somatostatin analogues reduce PDGF-induced endometrial cell proliferation and motility. Hum Reprod, 2012. 27(7): p. 2117-29.

275. Katz, A.M., et al., Astrocyte-specific expression patterns associated with the PDGF-induced glioma microenvironment. PLoS One, 2012. 7(2): p. e32453.

276. Jin, R.U. and J.C. Mills, RAB26 coordinates lysosome traffic and mitochondrial localization. J Cell Sci, 2014. 127(Pt 5): p. 1018-32.

277. Nawaz, M., et al., The emerging role of extracellular vesicles as biomarkers for urogenital cancers. Nat Rev Urol, 2014. 11(12): p. 688-701.

278. Nicholson, A.D., et al., Automated quantitative analysis of tissue microarray of 443 patients with colorectal adenocarcinoma: low expression of Bcl-2 predicts poor survival. J Am Coll Surg, 2014. 219(5): p. 977-87.

279. Chen, L., et al., Differential targeting of prosurvival BCl-2 proteins by their BH3-only ligands allows complementary apoptotic function. Mol Cell, 2005. 17(3): p. 393-403.

280. Akl, H., et al., A dual role for the anti-apoptotic Bcl-2 protein in cancer: mitochondria versus endoplasmic reticulum. Biochim Biophys Acta, 2014. 1843(10): p. 2240-52.

281. Greenhough, A., et al., The proapoptotic BH3-only protein Bim is downregulated in a subset of colorectal cancers and is repressed by antiapoptotic COX-2/PGE(2) signalling in colorectal adenoma cells. Oncogene, 2010. 29(23): p. 3398-410.

282. Mariani, L., et al., Glioma cell motility is associated with reduced transcription of proapoptotic and proliferation genes: a cDNA microarray analysis. J Neurooncol, 2001. 53(2): p. 161-76.

283. Cimmino, A., et al., miR-15 and miR-16 induce apoptosis by targeting BCL2. Proc Natl Acad Sci U S A, 2005. 102(39): p. 13944-9.

284. Liu, D., et al., The zinc-finger transcription factor, early growth response 3, mediates VEGFinduced angiogenesis. Oncogene, 2008. 27(21): p. 2989-98.

285. Urbich, C., et al., HDAC5 is a repressor of angiogenesis and determines the angiogenic gene expression pattern of endothelial cells. Blood, 2009. 113(22): p. 5669-79.

286. Hu, B.Y., et al., Human oligodendrocytes from embryonic stem cells: conserved SHH signaling networks and divergent FGF effects. Development, 2009. 136(9): p. 1443-52.

287. Chu, J.E., et al., Lung-derived factors mediate breast cancer cell migration through CD44 receptor-ligand interactions in a novel ex vivo system for analysis of organ-specific soluble proteins. Neoplasia, 2014. 16(2): p. 180-91.

288. Bourguignon, L.Y., W. Xia, and G. Wong, Hyaluronan-mediated CD44 interaction with p300 and SIRT1 regulates beta-catenin signaling and NFkappaB-specific transcription activity leading to MDR1 and Bcl-xL gene expression and chemoresistance in breast tumor cells. J Biol Chem, 2009. 284(5): p. 2657-71.

289. Shtivelman, E. and J.M. Bishop, Expression of CD44 is repressed in neuroblastoma cells. Mol Cell Biol, 1991. 11(11): p. 5446-53.

290. Leisewitz, A.V., et al., A PPARs cross-talk concertedly commits C6 glioma cells to oligodendrocytes and induces enzymes involved in myelin synthesis. J Cell Physiol, 2008. 217(2): p. 367-76.

291. Poliseno, L., et al., A coding-independent function of gene and pseudogene mRNAs regulates tumour biology. Nature, 2010. 465(7301): p. 1033-8.

292. Khan, I., et al., Registered report: A coding-independent function of gene and pseudogene mRNAs regulates tumour biology. Elife, 2015. 4. 
293. Michaud, K., et al., Contribution of $1 p, 19 q, 9 p$ and $10 q$ Automated Analysis by FISH to the Diagnosis and Prognosis of Oligodendroglial Tumors According to WHO 2016 Guidelines. PLoS One, 2016. 11(12): p. e0168728.

294. Duval, C., et al., ImmunoFISH is a reliable technique for the assessment of $1 p$ and $19 q$ status in oligodendrogliomas. PLoS One, 2014. 9(6): p. e100342.

295. Carter, J.H., et al., Targeted Next-Generation Sequencing in Molecular Subtyping of Lower-Grade Diffuse Gliomas: Application of the World Health Organization's 2016 Revised Criteria for Central Nervous System Tumors. J Mol Diagn, 2017. 19(2): p. 328-337.

296. Venteicher, A.S., et al., 142 Genetic and Nongenetic Determinants of Cellular Architecture in IDH1-Mutant Oligodendrogliomas and Astrocytomas Using Single-Cell Transcriptome Analysis. Neurosurgery, 2016. 63 Suppl 1: p. 158.

297. Tirosh, I., et al., Single-cell RNA-seq supports a developmental hierarchy in human oligodendroglioma. Nature, 2016. 539(7628): p. 309-313.

298. Venteicher, A.S., et al., Decoupling genetics, lineages, and microenvironment in IDH-mutant gliomas by single-cell RNA-seq. Science, 2017. 355(6332).

299. Harding, C., J. Heuser, and P. Stahl, Receptor-mediated endocytosis of transferrin and recycling of the transferrin receptor in rat reticulocytes. J Cell Biol, 1983. 97(2): p. 329-39.

300. Pan, B.T., et al., Electron microscopic evidence for externalization of the transferrin receptor in vesicular form in sheep reticulocytes. J Cell Biol, 1985. 101(3): p. 942-8.

301. Johnstone, R.M., et al., Vesicle formation during reticulocyte maturation. Association of plasma membrane activities with released vesicles (exosomes). J Biol Chem, 1987. 262(19): p. 9412-20.

302. Raposo, G., et al., B lymphocytes secrete antigen-presenting vesicles. J Exp Med, 1996. 183(3): $p$. 1161-72.

303. Zitvogel, L., et al., Eradication of established murine tumors using a novel cell-free vaccine: dendritic cell-derived exosomes. Nat Med, 1998. 4(5): p. 594-600.

304. Thery, C., et al., Molecular characterization of dendritic cell-derived exosomes. Selective accumulation of the heat shock protein hsc73. J Cell Biol, 1999. 147(3): p. 599-610.

305. Potolicchio, I., et al., Proteomic analysis of microglia-derived exosomes: metabolic role of the aminopeptidase CD13 in neuropeptide catabolism. J Immunol, 2005. 175(4): p. 2237-43.

306. Turola, E., et al., Microglial microvesicle secretion and intercellular signaling. Front Physiol, 2012. 3: p. 149.

307. Faure, J., et al., Exosomes are released by cultured cortical neurones. Mol Cell Neurosci, 2006. 31(4): p. 642-8.

308. Kapustin, A.N., et al., Vascular smooth muscle cell calcification is mediated by regulated exosome secretion. Circ Res, 2015. 116(8): p. 1312-23.

309. Thomou, T., et al., Adipose-derived circulating miRNAs regulate gene expression in other tissues. Nature, 2017. 542(7642): p. 450-455.

310. Andre, F., et al., Malignant effusions and immunogenic tumour-derived exosomes. Lancet, 2002. 360(9329): p. 295-305.

311. Nawaz, M., et al., Extracellular vesicles in ovarian cancer: applications to tumor biology, immunotherapy and biomarker discovery. Expert Rev Proteomics, 2016. 13(4): p. 395-409.

312. Fatima, F. and M. Nawaz, Stem cell-derived exosomes: roles in stromal remodeling, tumor progression, and cancer immunotherapy. Chin J Cancer, 2015. 34(12): p. 541-53.

313. Nawaz, M., et al., Extracellular Vesicles: Evolving Factors in Stem Cell Biology. Stem Cells Int, 2016. 2016: p. 1073140.

314. Samuel, M., et al., Extracellular vesicles including exosomes in cross kingdom regulation: $a$ viewpoint from plant-fungal interactions. Front Plant Sci, 2015. 6: p. 766. 
315. Oliviusson, P., et al., Plant retromer, localized to the prevacuolar compartment and microvesicles in Arabidopsis, may interact with vacuolar sorting receptors. Plant Cell, 2006. 18(5): p. 1239-52.

316. Regente, M., et al., Vesicular fractions of sunflower apoplastic fluids are associated with potential exosome marker proteins. FEBS Lett, 2009. 583(20): p. 3363-6.

317. Record, M., Exosome-like nanoparticles from food: protective nanoshuttles for bioactive cargo. Mol Ther, 2013. 21(7): p. 1294-6.

318. Rodrigues, M.L., et al., Extracellular vesicles produced by Cryptococcus neoformans contain protein components associated with virulence. Eukaryot Cell, 2008. 7(1): p. 58-67.

319. Albuquerque, P.C., et al., Vesicular transport in Histoplasma capsulatum: an effective mechanism for trans-cell wall transfer of proteins and lipids in ascomycetes. Cell Microbiol, 2008. 10(8): p. 1695-710.

320. Peres da Silva, R., et al., Extracellular vesicle-mediated export of fungal RNA. Sci Rep, 2015. 5: $\mathrm{p}$. 7763.

321. Kondo, K., A. Takade, and K. Amako, Release of the outer membrane vesicles from Vibrio cholerae and Vibrio parahaemolyticus. Microbiol Immunol, 1993. 37(2): p. 149-52.

322. Beveridge, T.J., Structures of gram-negative cell walls and their derived membrane vesicles. J Bacteriol, 1999. 181(16): p. 4725-33.

323. Lee, E.Y., et al., Gram-positive bacteria produce membrane vesicles: proteomics-based characterization of Staphylococcus aureus-derived membrane vesicles. Proteomics, 2009. 9(24): p. 5425-36.

324. Gould, S.J., A.M. Booth, and J.E. Hildreth, The Trojan exosome hypothesis. Proc Natl Acad Sci U S A, 2003. 100(19): p. 10592-7.

325. Masciopinto, F., et al., Association of hepatitis $C$ virus envelope proteins with exosomes. Eur J Immunol, 2004. 34(10): p. 2834-42.

326. Pelchen-Matthews, A., G. Raposo, and M. Marsh, Endosomes, exosomes and Trojan viruses. Trends Microbiol, 2004. 12(7): p. 310-6.

327. Pegtel, D.M., et al., Functional delivery of viral miRNAs via exosomes. Proc Natl Acad Sci U S A, 2010. 107(14): p. 6328-33.

328. Goncalves, M.F., et al., Trypanosoma cruzi: shedding of surface antigens as membrane vesicles. Exp Parasitol, 1991. 72(1): p. 43-53.

329. Trocoli Torrecilhas, A.C., et al., Trypanosoma cruzi: parasite shed vesicles increase heart parasitism and generate an intense inflammatory response. Microbes Infect, 2009. 11(1): p. 2939.

330. Nogueira, P.M., et al., Vesicles from different Trypanosoma cruzi strains trigger differential innate and chronic immune responses. J Extracell Vesicles, 2015. 4: p. 28734.

331. Martin-Jaular, L., et al., Exosomes from Plasmodium yoelii-infected reticulocytes protect mice from lethal infections. PLoS One, 2011. 6(10): p. e26588.

332. Mantel, P.Y., et al., Malaria-infected erythrocyte-derived microvesicles mediate cellular communication within the parasite population and with the host immune system. Cell Host Microbe, 2013. 13(5): p. 521-34.

333. Caby, M.P., et al., Exosomal-like vesicles are present in human blood plasma. Int Immunol, 2005. 17(7): p. 879-87.

334. Skog, J., et al., Glioblastoma microvesicles transport RNA and proteins that promote tumour growth and provide diagnostic biomarkers. Nat Cell Biol, 2008. 10(12): p. 1470-6.

335. Pisitkun, T., R.F. Shen, and M.A. Knepper, Identification and proteomic profiling of exosomes in human urine. Proc Natl Acad Sci U S A, 2004. 101(36): p. 13368-73.

336. Admyre, C., et al., Exosomes with immune modulatory features are present in human breast milk. J Immunol, 2007. 179(3): p. 1969-78. 
337. Ogawa, Y., et al., Exosome-like vesicles with dipeptidyl peptidase IV in human saliva. Biol Pharm Bull, 2008. 31(6): p. 1059-62.

338. Michael, A., et al., Exosomes from human saliva as a source of microRNA biomarkers. Oral Dis, 2010. 16(1): p. 34-8.

339. Lasser, C., et al., Human saliva, plasma and breast milk exosomes contain RNA: uptake by macrophages. J Transl Med, 2011. 9: p. 9.

340. Akers, J.C., et al., miRNA contents of cerebrospinal fluid extracellular vesicles in glioblastoma patients. J Neurooncol, 2015. 123(2): p. 205-16.

341. Prado, N., et al., Exosomes from bronchoalveolar fluid of tolerized mice prevent allergic reaction. J Immunol, 2008. 181(2): p. 1519-25.

342. Qazi, K.R., et al., Proinflammatory exosomes in bronchoalveolar lavage fluid of patients with sarcoidosis. Thorax, 2010. 65(11): p. 1016-24.

343. Skriner, K., et al., Association of citrullinated proteins with synovial exosomes. Arthritis Rheum, 2006. 54(12): p. 3809-14.

344. Asea, A., et al., Heat shock protein-containing exosomes in mid-trimester amniotic fluids. J Reprod Immunol, 2008. 79(1): p. 12-7.

345. Poliakov, A., et al., Structural heterogeneity and protein composition of exosome-like vesicles (prostasomes) in human semen. Prostate, 2009. 69(2): p. 159-67.

346. Minciacchi, V.R., M.R. Freeman, and D. Di Vizio, Extracellular vesicles in cancer: exosomes, microvesicles and the emerging role of large oncosomes. Semin Cell Dev Biol, 2015. 40: p. 41-51.

347. Van Giau, V. and S.S. An, Emergence of exosomal miRNAs as a diagnostic biomarker for Alzheimer's disease. J Neurol Sci, 2016. 360: p. 141-52.

348. Keerthikumar, S., et al., ExoCarta: A Web-Based Compendium of Exosomal Cargo. J Mol Biol, 2016. 428(4): p. 688-92.

349. Balaj, L., et al., Tumour microvesicles contain retrotransposon elements and amplified oncogene sequences. Nat Commun, 2011. 2: p. 180.

350. Waldenstrom, A., et al., Cardiomyocyte microvesicles contain DNA/RNA and convey biological messages to target cells. PLoS One, 2012. 7(4): p. e34653.

351. Lee, T.H., et al., Oncogenic ras-driven cancer cell vesiculation leads to emission of doublestranded DNA capable of interacting with target cells. Biochem Biophys Res Commun, 2014. 451(2): p. 295-301.

352. Thakur, B.K., et al., Double-stranded DNA in exosomes: a novel biomarker in cancer detection. Cell Res, 2014. 24(6): p. 766-9.

353. Lazaro-Ibanez, E., et al., Different gDNA content in the subpopulations of prostate cancer extracellular vesicles: apoptotic bodies, microvesicles, and exosomes. Prostate, 2014. 74(14): p. 1379-90.

354. Guescini, M., et al., Astrocytes and Glioblastoma cells release exosomes carrying mtDNA. J Neural Transm (Vienna), 2010. 117(1): p. 1-4.

355. Guescini, M., et al., C2C12 myoblasts release micro-vesicles containing mtDNA and proteins involved in signal transduction. Exp Cell Res, 2010. 316(12): p. 1977-84.

356. Ratajczak, J., et al., Embryonic stem cell-derived microvesicles reprogram hematopoietic progenitors: evidence for horizontal transfer of mRNA and protein delivery. Leukemia, 2006. 20(5): p. 847-56.

357. Valadi, H., et al., Exosome-mediated transfer of mRNAs and microRNAs is a novel mechanism of genetic exchange between cells. Nat Cell Biol, 2007. 9(6): p. 654-9.

358. Fatima, F. and M. Nawaz, Vesiculated Long Non-Coding RNAs: Offshore Packages Deciphering Trans-Regulation between Cells, Cancer Progression and Resistance to Therapies. Non-Coding RNA, 2017. 3(1): p. 10. 
359. Kalra, H., et al., Vesiclepedia: a compendium for extracellular vesicles with continuous community annotation. PLoS Biol, 2012. 10(12): p. e1001450.

360. Kim, D.K., et al., EVpedia: a community web portal for extracellular vesicles research. Bioinformatics, 2015. 31(6): p. 933-9.

361. Kim, D.K., et al., EVpedia: A community web resource for prokaryotic and eukaryotic extracellular vesicles research. Semin Cell Dev Biol, 2015. 40: p. 4-7.

362. Deregibus, M.C., et al., Endothelial progenitor cell derived microvesicles activate an angiogenic program in endothelial cells by a horizontal transfer of mRNA. Blood, 2007. 110(7): p. 2440-8.

363. Al-Nedawi, K., et al., Intercellular transfer of the oncogenic receptor EGFRvIII by microvesicles derived from tumour cells. Nat Cell Biol, 2008. 10(5): p. 619-24.

364. Montecalvo, A., et al., Mechanism of transfer of functional microRNAs between mouse dendritic cells via exosomes. Blood, 2012. 119(3): p. 756-66.

365. Gardiner, C., et al., Techniques used for the isolation and characterization of extracellular vesicles: results of a worldwide survey. J Extracell Vesicles, 2016. 5: p. 32945.

366. Mateescu, B., et al., Obstacles and opportunities in the functional analysis of extracellular vesicle RNA - an ISEV position paper. Journal of Extracellular Vesicles, 2017. 6(1): p. 1286095.

367. Witwer, K.W., et al., Standardization of sample collection, isolation and analysis methods in extracellular vesicle research. J Extracell Vesicles, 2013. 2.

368. Sodar, B.W., et al., Low-density lipoprotein mimics blood plasma-derived exosomes and microvesicles during isolation and detection. Sci Rep, 2016. 6: p. 24316.

369. Rupert, D.L., et al., Methods for the physical characterization and quantification of extracellular vesicles in biological samples. Biochim Biophys Acta, 2017. 1861(1 Pt A): p. 3164-3179.

370. Maas, S.L., et al., Possibilities and limitations of current technologies for quantification of biological extracellular vesicles and synthetic mimics. J Control Release, 2015. 200: p. 87-96.

371. Baldwin, S., et al., Analyzing the miRNA content of extracellular vesicles by fluorescence nanoparticle tracking. Nanomedicine, 2016. 13(2): p. 765-770.

372. Dragovic, R.A., et al., Sizing and phenotyping of cellular vesicles using Nanoparticle Tracking Analysis. Nanomedicine, 2011. 7(6): p. 780-8.

373. Gardiner, C., et al., Extracellular vesicle sizing and enumeration by nanoparticle tracking analysis. J Extracell Vesicles, 2013. 2.

374. Pachler, K., et al., A Good Manufacturing Practice-grade standard protocol for exclusively human mesenchymal stromal cell-derived extracellular vesicles. Cytotherapy, 2017.

375. Kibria, G., et al., A rapid, automated surface protein profiling of single circulating exosomes in human blood. Sci Rep, 2016. 6: p. 36502.

376. Nardi Fda, S., et al., High levels of circulating extracellular vesicles with altered expression and function during pregnancy. Immunobiology, 2016. 221(7): p. 753-60.

377. van der Pol, E., et al., Particle size distribution of exosomes and microvesicles determined by transmission electron microscopy, flow cytometry, nanoparticle tracking analysis, and resistive pulse sensing. J Thromb Haemost, 2014. 12(7): p. 1182-92.

378. Dragovic, R.A., et al., Isolation of syncytiotrophoblast microvesicles and exosomes and their characterisation by multicolour flow cytometry and fluorescence Nanoparticle Tracking Analysis. Methods, 2015. 87: p. 64-74.

379. Vogel, R., et al., A standardized method to determine the concentration of extracellular vesicles using tunable resistive pulse sensing. J Extracell Vesicles, 2016. 5: p. 31242.

380. Fertig, E.T., M. Gherghiceanu, and L.M. Popescu, Extracellular vesicles release by cardiac telocytes: electron microscopy and electron tomography. J Cell Mol Med, 2014. 18(10): p. 193843. 
381. Brisson, A.R., et al., Extracellular vesicles from activated platelets: a semiquantitative cryoelectron microscopy and immuno-gold labeling study. Platelets, 2017: p. 1-9.

382. Hoog, J.L. and J. Lotvall, Diversity of extracellular vesicles in human ejaculates revealed by cryoelectron microscopy. J Extracell Vesicles, 2015. 4: p. 28680.

383. Groot Kormelink, T., et al., Prerequisites for the analysis and sorting of extracellular vesicle subpopulations by high-resolution flow cytometry. Cytometry A, 2016. 89(2): p. 135-47.

384. van der Vlist, E.J., et al., Fluorescent labeling of nano-sized vesicles released by cells and subsequent quantitative and qualitative analysis by high-resolution flow cytometry. Nat Protoc, 2012. 7(7): p. 1311-26.

385. Nolan, J.P., Flow Cytometry of Extracellular Vesicles: Potential, Pitfalls, and Prospects. Curr Protoc Cytom, 2015. 73: p. 1314 1-16.

386. Reis, F.C., et al., Fat-specific Dicer deficiency accelerates aging and mitigates several effects of dietary restriction in mice. Aging (Albany NY), 2016. 8(6): p. 1201-22.

387. Lasser, C., et al., Two distinct extracellular RNA signatures released by a single cell type identified by microarray and next-generation sequencing. RNA Biol, 2017. 14(1): p. 58-72.

388. Lunavat, T.R., et al., Small RNA deep sequencing discriminates subsets of extracellular vesicles released by melanoma cells--Evidence of unique microRNA cargos. RNA Biol, 2015. 12(8): p. 81023.

389. Crescitelli, R., et al., Distinct RNA profiles in subpopulations of extracellular vesicles: apoptotic bodies, microvesicles and exosomes. J Extracell Vesicles, 2013. 2.

390. Conley, A., et al., High-throughput sequencing of two populations of extracellular vesicles provides an mRNA signature that can be detected in the circulation of breast cancer patients. RNA Biol, 2016: p. 0.

391. Huang, X., et al., Characterization of human plasma-derived exosomal RNAs by deep sequencing. BMC Genomics, 2013. 14: p. 319.

392. Arroyo, J.D., et al., Argonaute2 complexes carry a population of circulating microRNAs independent of vesicles in human plasma. Proc Natl Acad Sci U S A, 2011. 108(12): p. 5003-8.

393. Turchinovich, A., et al., Characterization of extracellular circulating microRNA. Nucleic Acids Res, 2011. 39(16): p. 7223-33.

394. Gourzones, C., et al., Consistent high concentration of the viral microRNA BART17 in plasma samples from nasopharyngeal carcinoma patients--evidence of non-exosomal transport. Virol J, 2013. 10: p. 119.

395. Chevillet, J.R., et al., Quantitative and stoichiometric analysis of the microRNA content of exosomes. Proc Natl Acad Sci U S A, 2014. 111(41): p. 14888-93.

396. Wei, Z., et al., Fetal Bovine Serum RNA Interferes with the Cell Culture derived Extracellular RNA. Sci Rep, 2016. 6: p. 31175.

397. Tosar, J.P., et al., Ribonucleic artefacts: are some extracellular RNA discoveries driven by cell culture medium components? Journal of Extracellular Vesicles, 2017. 6(1): p. 1272832.

398. Simons, M. and G. Raposo, Exosomes--vesicular carriers for intercellular communication. Curr Opin Cell Biol, 2009. 21(4): p. 575-81.

399. Mathivanan, S., H. Ji, and R.J. Simpson, Exosomes: extracellular organelles important in intercellular communication. J Proteomics, 2010. 73(10): p. 1907-20.

400. Raposo, G. and W. Stoorvogel, Extracellular vesicles: exosomes, microvesicles, and friends. J Cell Biol, 2013. 200(4): p. 373-83.

401. Hoshino, A., et al., Tumour exosome integrins determine organotropic metastasis. Nature, 2015. 527(7578): p. 329-35.

402. Yuana, Y., A. Sturk, and R. Nieuwland, Extracellular vesicles in physiological and pathological conditions. Blood Rev, 2013. 27(1): p. 31-9. 
403. Sullivan, R., et al., Role of exosomes in sperm maturation during the transit along the male reproductive tract. Blood Cells Mol Dis, 2005. 35(1): p. 1-10.

404. Tannetta, D., et al., Extracellular vesicles and reproduction-promotion of successful pregnancy. Cell Mol Immunol, 2014. 11(6): p. 548-63.

405. Schiera, G., C.M. Di Liegro, and I. Di Liegro, Extracellular Membrane Vesicles as Vehicles for Brain Cell-to-Cell Interactions in Physiological as well as Pathological Conditions. Biomed Res Int, 2015. 2015: p. 152926.

406. Lai, C.P. and X.O. Breakefield, Role of exosomes/microvesicles in the nervous system and use in emerging therapies. Front Physiol, 2012. 3: p. 228.

407. Fruhbeis, C., D. Frohlich, and E.M. Kramer-Albers, Emerging roles of exosomes in neuron-glia communication. Front Physiol, 2012. 3: p. 119.

408. Chivet, M., et al., Emerging role of neuronal exosomes in the central nervous system. Front Physiol, 2012. 3: p. 145.

409. Pegtel, D.M., L. Peferoen, and S. Amor, Extracellular vesicles as modulators of cell-to-cell communication in the healthy and diseased brain. Philos Trans R Soc Lond B Biol Sci, 2014. 369(1652).

410. Rajendran, L., et al., Emerging roles of extracellular vesicles in the nervous system. J Neurosci, 2014. 34(46): p. 15482-9.

411. Zappulli, V., et al., Extracellular vesicles and intercellular communication within the nervous system. J Clin Invest, 2016. 126(4): p. 1198-207.

412. Rajendran, L., et al., Alzheimer's disease beta-amyloid peptides are released in association with exosomes. Proc Natl Acad Sci U S A, 2006. 103(30): p. 11172-7.

413. Vella, L.J., et al., The role of exosomes in the processing of proteins associated with neurodegenerative diseases. Eur Biophys J, 2008. 37(3): p. 323-32.

414. Bellingham, S.A., et al., Exosomes: vehicles for the transfer of toxic proteins associated with neurodegenerative diseases? Front Physiol, 2012. 3: p. 124.

415. Buzas, E.I., et al., Emerging role of extracellular vesicles in inflammatory diseases. Nat Rev Rheumatol, 2014. 10(6): p. 356-64.

416. Osteikoetxea, X., et al., Extracellular vesicles in cardiovascular disease: are they Jedi or Sith? J Physiol, 2016. 594(11): p. 2881-94.

417. Evans-Osses, I., L.H. Reichembach, and M.I. Ramirez, Exosomes or microvesicles? Two kinds of extracellular vesicles with different routes to modify protozoan-host cell interaction. Parasitol Res, 2015. 114(10): p. 3567-75.

418. Fatima, F. and M. Nawaz, Nexus between extracellular vesicles, immunomodulation and tissue remodeling: for good or for bad? Annals of Translational Medicine, 2017.

419. Nawaz, M., et al., Microvesicles in Gliomas and Medulloblastomas: An Overview. Journal of Cancer Therapy, 2014. 5(2): p. 10.

420. Al-Nedawi, K., et al., Endothelial expression of autocrine VEGF upon the uptake of tumor-derived microvesicles containing oncogenic EGFR. Proc Natl Acad Sci U S A, 2009. 106(10): p. 3794-9.

421. Peinado, H., et al., Melanoma exosomes educate bone marrow progenitor cells toward a prometastatic phenotype through MET. Nat Med, 2012. 18(6): p. 883-91.

422. Greening, D.W., et al., Secreted primary human malignant mesothelioma exosome signature reflects oncogenic cargo. Sci Rep, 2016. 6: p. 32643.

423. Di Vizio, D., et al., Large oncosomes in human prostate cancer tissues and in the circulation of mice with metastatic disease. Am J Pathol, 2012. 181(5): p. 1573-84.

424. Morello, M., et al., Large oncosomes mediate intercellular transfer of functional microRNA. Cell Cycle, 2013. 12(22): p. 3526-36. 
425. Zhang, L., et al., Microenvironment-induced PTEN loss by exosomal microRNA primes brain metastasis outgrowth. Nature, 2015. 527(7576): p. 100-4.

426. Chinese Journal of, C., The 150 most important questions in cancer research and clinical oncology series: questions 15-24 : Edited by Chinese Journal of Cancer. Chin J Cancer, 2017. 36(1): p. 39.

427. Janowska-Wieczorek, A., et al., Microvesicles derived from activated platelets induce metastasis and angiogenesis in lung cancer. Int J Cancer, 2005. 113(5): p. 752-60.

428. Janowska-Wieczorek, A., et al., Enhancing effect of platelet-derived microvesicles on the invasive potential of breast cancer cells. Transfusion, 2006. 46(7): p. 1199-209.

429. Costa-Silva, B., et al., Pancreatic cancer exosomes initiate pre-metastatic niche formation in the liver. Nat Cell Biol, 2015. 17(6): p. 816-26.

430. Lima, L.G., et al., Tumor-derived microvesicles modulate the establishment of metastatic melanoma in a phosphatidylserine-dependent manner. Cancer Lett, 2009. 283(2): p. 168-75.

431. Greening, D.W., et al., Emerging roles of exosomes during epithelial-mesenchymal transition and cancer progression. Semin Cell Dev Biol, 2015.

432. Taverna, S., et al., Exosomal shuttling of miR-126 in endothelial cells modulates adhesive and migratory abilities of chronic myelogenous leukemia cells. Mol Cancer, 2014. 13: p. 169.

433. Graves, L.E., et al., Proinvasive properties of ovarian cancer ascites-derived membrane vesicles. Cancer Res, 2004. 64(19): p. 7045-9.

434. Taylor, D.D. and C. Gercel-Taylor, MicroRNA signatures of tumor-derived exosomes as diagnostic biomarkers of ovarian cancer. Gynecol Oncol, 2008. 110(1): p. 13-21.

435. Rank, A., et al., Circulating microparticles in patients with benign and malignant ovarian tumors. Anticancer Res, 2012. 32(5): p. 2009-14.

436. Kuhlmann, J.D., et al., microRNA and the pathogenesis of ovarian cancer--a new horizon for molecular diagnostics and treatment? Clin Chem Lab Med, 2012. 50(4): p. 601-15.

437. Larssen, P., et al., Tracing Cellular Origin of Human Exosomes Using Multiplex Proximity Extension Assay. Mol Cell Proteomics, 2017.

438. Kahlert, C., et al., Identification of double-stranded genomic DNA spanning all chromosomes with mutated KRAS and p53 DNA in the serum exosomes of patients with pancreatic cancer. J Biol Chem, 2014. 289(7): p. 3869-75.

439. Brock G, C.-R.E., Hu L, Coticchia C, Skog J. , Liquid biopsy for cancer screening, patient stratification and monitoring. Transl Cancer Res, 2015. 4(3): p. 280-290.

440. Royo, F., et al., Comparative miRNA Analysis of Urine Extracellular Vesicles Isolated through Five Different Methods. Cancers (Basel), 2016. 8(12).

441. Krafft, C., et al., A specific spectral signature of serum and plasma-derived extracellular vesicles for cancer screening. Nanomedicine, 2016.

442. Cappello, F., et al., Exosome levels in human body fluids: A tumor marker by themselves? Eur J Pharm Sci, 2017. 96: p. 93-98.

443. Fatima, F., J. Squire, and M. Nawaz, Mining extracellular vesicles for clinically relevant noninvasive diagnostic biomarkers in cancer, J. Wang, Editor. 2017, InTechOpen.

444. Kim, M.S., et al., Development of exosome-encapsulated paclitaxel to overcome MDR in cancer cells. Nanomedicine, 2016. 12(3): p. 655-64.

445. Kordelas, L., et al., MSC-derived exosomes: a novel tool to treat therapy-refractory graft-versushost disease. Leukemia, 2014. 28(4): p. 970-3.

446. Lener, T., et al., Applying extracellular vesicles based therapeutics in clinical trials - an ISEV position paper. J Extracell Vesicles, 2015. 4: p. 30087.

447. Fais, S., et al., Evidence-Based Clinical Use of Nanoscale Extracellular Vesicles in Nanomedicine. ACS Nano, 2016. 10(4): p. 3886-99. 
448. Wahlgren, J., et al., Plasma exosomes can deliver exogenous short interfering RNA to monocytes and lymphocytes. Nucleic Acids Res, 2012. 40(17): p. e130.

449. Alvarez-Erviti, L., et al., Delivery of siRNA to the mouse brain by systemic injection of targeted exosomes. Nat Biotechnol, 2011. 29(4): p. 341-5.

450. Eldh, M., et al., Importance of RNA isolation methods for analysis of exosomal RNA: evaluation of different methods. Mol Immunol, 2012. 50(4): p. 278-86.

451. Sinha, A., et al., In-depth proteomic analyses of ovarian cancer cell line exosomes reveals differential enrichment of functional categories compared to the $\mathrm{NCl} 60$ proteome. Biochem Biophys Res Commun, 2014. 445(4): p. 694-701.

452. Principe, S., et al., In-depth proteomic analyses of exosomes isolated from expressed prostatic secretions in urine. Proteomics, 2013. 13(10-11): p. 1667-71.

453. Villarroya-Beltri, C., et al., Sumoylated hnRNPA2B1 controls the sorting of miRNAs into exosomes through binding to specific motifs. Nat Commun, 2013. 4: p. 2980.

454. McKenzie, A.J., et al., KRAS-MEK Signaling Controls Ago2 Sorting into Exosomes. Cell Rep, 2016. 15(5): p. 978-87.

455. Shurtleff, M.J., et al., Y-box protein 1 is required to sort microRNAs into exosomes in cells and in a cell-free reaction. Elife, 2016. 5.

456. Fuster-Matanzo, A., et al., Acellular approaches for regenerative medicine: on the verge of clinical trials with extracellular membrane vesicles? Stem Cell Res Ther, 2015. 6: p. 227. 


\subsection{Electronic Databases}

- Ariadne pathways analysis software - http://www.ariadnegenomics.com/products

- Bioconductor - https://www.bioconductor.org/

- DIANA tools - http://diana.imis.athena-innovation.gr/DianaTools/index.php?r=site/index

- Ensembl genome browser - http://www.ensembl.org/index.html

- EvPedia: Extracellular vesicles database - http://student4.postech.ac.kr/evpedia2_xe/xe/

- EV-Track platform for extracellular vesicles reporting - http://evtrack.org/

- Exocarta: Exosome database - http://www.exocarta.org/

- GenBank NCBI - https://www.ncbi.nlm.nih.gov/genbank/

- ISEV: Vesicles community - http://www.isev.org/

- miRBase: microRNA database - http://www.mirbase.org/

- $\operatorname{miRNA-database~-~http://www.microrna.org/microrna/getDownloads.do~}$

- $\quad \operatorname{mirSVR}$ - www.microRNA.org

- NCBI PubMed - https://www.ncbi.nlm.nih.gov/pubmed

- PicTar - http://pictar.mdc-berlin.de/

- $\mathrm{R}$ - Environment/Package - https://cran.r-project.org/

- SEER Cancer Statistics - https://seer.cancer.gov/archive/csr/1975_2013/

- TargetScan - http://www.targetscan.org/vert_71/

- UCSC Genome Browser - https://genome.ucsc.edu/

- Vesiclepedia: Microvesicles database - http://www.microvesicles.org/

- Wikipedia - https://www.wikipedia.org/ 


\section{ANNEX}

\section{Annex A}

Ethical approval certificate

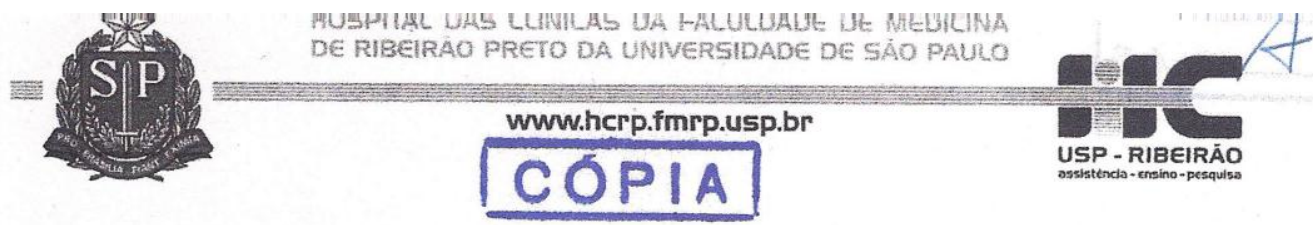

Ribeirão Preto, 19 de agosto de 2008

Oficio $n^{\circ} 3045 / 2008$

$\mathrm{CEP} / \mathrm{MGV}$

Prezado Senhor,

O trabalho intitulado "ESTUDO DA EXPRESSÃO DE microRNAS E mRNAS EM OLIGODENDROGLIOMAS DE DIFERENTES GRAUS DE MALIGNIDADE", foi analisado pelo Comitê de Ética em Pesquisa, em sua $272^{a}$ Reunião Ordinária realizada em 18/08/2008 e enquadrado na categoria: 1 PROVADO, de acordo com o Processo HCRP $n^{\circ}$ $8730 / 2008$.

Este Comitê sègue integralmente a Conferência Internacional de Harmonização de Boas Práticas Clínicas (IGH-GCP), bem como a Resolução $n^{\circ}$ 196/96 CNS/MS.

Lembramos que devem ser apresentados a este CEP, O Relatório Parcial e o Relatório Final da pesquisa.

Atenciosamente.

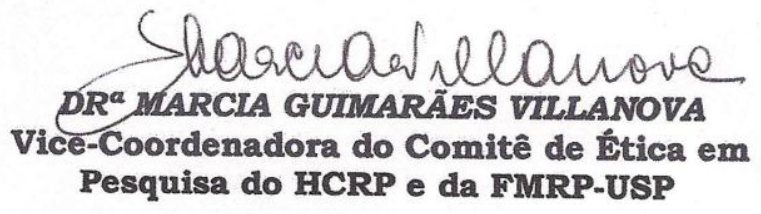

Ilustrissimo Senhor

LUCIANO NEDER SERAFINI

Depto. de Patologia 


\section{Annex B}

\section{List of Publications, Book Chapter and Editorials}

\section{Publications}

1. Muhammad Nawaz, Giovanni Camussi, Hadi Valadi, Irina Nazarenko, Karin Ekström, Xiaoqin Wang, Simona Principe, Neelam Shah, Naeem M Ashraf, Farah Fatima, Luciano Neder, Thomas Kislinger*. The emerging role of extracellular vesicles as biomarkers for urogenital cancers, Nature Reviews Urology, 2014, 11(12): 688-701.

2. Muhammad Nawaz, Fatima F, Zanetti BR, de Lima Martins I, Schiavotelo LN, Mendes ND, Silvestre RN, Luciano Neder*. Microvesicles in Gliomas and Medulloblastomas: An Overview, Journal of Cancer Therapy, 2014. 5(2): 182-191.

3. Farah Fatima and Muhammad Nawaz*. Stem cell-derived exosomes: roles in stromal remodeling, tumor progression, and cancer immunotherapy. Chinese Journal of Cancer, 2015, 34(46): 1-13.

4. Muhammad Nawaz*, Farah Fatima, Krishna C. Vallabhaneni, Patrice Penfornis, Hadi Valadi, Karin Ekström, Sharad Kholia, Jason D. Whitt, Joseph D. Fernandes, Radhika Pochampally, Jeremy A. Squire, Giovanni Camussi*. Extracellular Vesicles: Evolving Factors in Stem Cell Biology, Stem Cells International, 2016, (2016): 1073140.

5. Muhammad Nawaz*, Farah Fatima, Irina Nazarenko, Karin Ekström, Iram Murtaza, Mariam Anees, Aneesa Sultan, Luciano Neder, Giovanni Camussi, Hadi Valadi, Jeremy Squire, Thomas Kislinger*. Extracellular vesicles in ovarian cancer: applications to tumor biology, immunotherapy and biomarker discovery. Expert Review of Proteomics, 2016, 13(4): 395-409.

6. Farah Fatima, Ying Fei, Abukar Ali, Majd Mohammad, Malin C. Erlandsson, Maria I. Bokarewa, Muhammad Nawaz, Hadi Valadi, Manli Na, Tao Jin *. Radiological features of experimental staphylococcal septic arthritis by micro computed tomography scan. PLoS One. 2017, 12(2):e0171222.

7. Bogdan Mateescu, Emma Kowal, Bas van Balkom, Sabine Bartel, Suvendra Bhattacharyya, Edit Buzas, Amy Buck, Paola di Candia, Franklin Chow, Saumya Das, Tom Driedonks, Lola Fernández-Messina, Franziska Haderk, Andrew Hill, Jennifer Jones, Kendall van KeurenJensen, Charles Lai, Cecilia Lässer, Italia di Liegro, Taral Lunavat, Magdalena Lorenowicz, Sybren Maas, Imre Mäger, Maria Mittelbrunn, Stefan Momma, Kamalika Mukherjee, Muhammed Nawaz, Dirk Pegtel, Michael Pfaffl, Raymond Schiffelers, Hidetoshi Tahara, Clotilde Thery, Juan Pablo Tosar, Marca Wauben, Kenneth Witwer, Esther Nolte-'t Hoen. 
Obstacles and opportunities in the functional analysis of extracellular vesicle RNA - an ISEV position paper. Journal of extracellular vesicles, 2017, 6(1):1286095.

8. Farah Fatima, Muhammad Nawaz*. Vesiculated Long Non-Coding RNAs: Offshore Packages Deciphering Trans-Regulation between Cells, Cancer Progression and Resistance to Therapies. Non-Coding RNA, 2017, 3 (1): 10.

Original article - Submitted

9. Hadi Valadi* et al. Mechanisms of RNA packaging into exosomes: Role of RNA binding proteins (2017).

10. Muhammad Nawaz and Luciano Neder* et al. MicroRNAs and mRNA expression profiling in grade III and grade II oligodendrogliomas (2017), (in process) - from PHD thesis. 


\section{Editorials}

1. Chinese Journal of Cancer, Chao-Nan Qian, Francesco Pezzella, Saverio Alberti, Manigreeva Krishnatreya, Muhammad Nawaz, Mei Wang, Shuai Chen. The 150 most important questions in cancer research and clinical oncology series: questions 15-24: Edited by Chinese Journal of Cancer. Chinese Journal of Cancer, 2017, 36(1):39.

2. Farah Fatima, Muhammad Nawaz*. Nexus between extracellular vesicles, immunomodulation and tissue remodeling: for good or for bad? Annals of Translational Medicine, 2017, 5(6):139. 


\section{Book Chapter (Accepted)}

Fatima, $\mathrm{F}^{1}$., JA. Squire ${ }^{1,2}$, and M. Nawaz ${ }^{1 *}$, Mining extracellular vesicles for clinically relevant non-invasive diagnostic biomarkers in cancer, in Novel Implications of Exosomes in Diagnosis and Treatment of Cancer, J. Wang, Editor. 2017, InTechOpen.

dx.doi.org/10.5772/intechopen.69406

\section{Authors affiliations}

1 Department of Pathology and Forensic Medicine, Ribeirão Preto Medical School, University of São Paulo, 14049900 / Ribeirão Preto - Brazil

2 Department of Genetics, Ribeirão Preto Medical School, University of São Paulo, 14049-900 / Ribeirão Preto Brazil

\section{Correspondence to;}

Muhammad Nawaz

Department of Pathology and Forensic Medicine, Ribeirão Preto Medical School, University of São Paulo, Av. Bandeirantes 3900, Ribeirão Preto 14049900 - SP

nawazm.edu@gmail.com 


\section{Annex C}

\section{Abstracts published in conference proceedings}

1. Luciano Neder, Benilton Carvalho, Muhammad Nawaz, Farah Fatima, VC de Oliveira, H Lima, R Haddad, RA Panepucci, Gilberto Carlotti Jr. MicroRNAs and gene expression profile differentially expressed in grade III and grade II oligodendrogliomas. Brain Pathology, v. 24. p. 95. September, 2014.

2. Muhammad Nawaz, Tina Rødgaard Højbøge, Marco Maugeri, Matin Mehryar, Klaus Villemoes, Peter MH Heegaard, Henrik Callesen, Hadi Valadi*. Profiling and characterization of embryo and seminal fluid extracellular vesicle-associated microRNAs in Sus scrofa: a possible role in differentiation and developmental communication processes. Journal of extracellular vesicles, 2016, 5(5).

3. Muhammad Nawaz, Jessica Wahlgren, Luisa Statello, Marco Maugeri, Alexandros Papadimitrio, Hadi Valadi*. Secretion of Toll-like receptor mRNAs via exosomes: a possible way of communicating messages against pathogens. Journal of extracellular vesicles, 2017, 6.

4. Farah Fatima, Majd Mohammad, Abukar Ali, Muhammad Nawaz, Hadi Valadi, Manli Na, Tao Jin. The inflammatory and immunological roles of $S$. aureus derived exosome-like vesicles in septic arthritis. Journal of extracellular vesicles, 2017, 6. 